\title{
An upper bound for the crossing number of augmented cubes *
}

\author{
Guoqing Wang ${ }^{a}$, Haoli Wang ${ }^{b}$, Yuansheng Yang ${ }^{b, \dagger}$ Xuezhi Yang ${ }^{b}$, Wenping Zheng ${ }^{c}$ \\ ${ }^{a}$ Department of Mathematics,
} \author{
Tianjin Polytechnic University, Tianjin 300387, China \\ ${ }^{b}$ Department of Computer Science, \\ Dalian University of Technology, Dalian 116024, China

\begin{abstract}
${ }^{c}$ Key Laboratory of Computational Intelligence and Chinese Information
Processing of Ministry of Education, Shanxi University, Taiyuan 030006, China
\end{abstract}

\begin{abstract}
A good drawing of a graph $G$ is a drawing where the edges are non-self-intersecting and each two edges have at most one point in common, which is either a common end vertex or a crossing. The crossing number of a graph $G$ is the minimum number of pairwise intersections of edges in a good drawing of $G$ in the plane. The $n$-dimensional augmented cube $A Q_{n}$, proposed by S.A. Choudum and V. Sunitha, is an important interconnection network with good topological properties and applications. In this paper, we obtain an upper bound on the crossing number of $A Q_{n}$ less than $\frac{26}{32} 4^{n}-\left(2 n^{2}+\frac{7}{2} n-6\right) 2^{n-2}$.
\end{abstract}

Keywords: Drawing; Crossing number; Augmented cube; Hypercube; Interconnection network

\section{Introduction}

Let $G$ be a simple connected graph with vertex set $V(G)$ and edge set $E(G)$. The crossing number $\operatorname{cr}(G)$ of a graph $G$ is the minimum possible number of edge crossings in a drawing of $G$ in the plane. The notion of crossing number is a central one for Topological Graph Theory and has been studied extensively by mathematicians including Erdős, Guy, Turán and Tutte, et al. (see [8, 12, 29, 30]). The study of crossing number not only is of theoretical importance, but also has many applications in, for example, VLSI theory and wiring layout problems (see [1, 19, 20, 27]).

However, the investigation on the crossing number of graphs is an extremely difficult problem. In 1973, Erdős and Guy [8] wrote, "Almost all questions that one can ask about crossing numbers remain unsolved." Actually, Garey and Johnson 11] proved that computing the crossing number is NP-complete. Not surprisingly, there are only a few infinite families of graphs for which the exact crossing numbers are known (see for example [22, 25, 26] ). Therefore, it is more practical to determine the upper and lower bounds of the crossing number of a graph. In particular, the bounds of crossing number of some popular parallel network topologies with good topological properties and applications in VLSI theory would be of theoretical importance and practical value. Among all the network topologies, the hypercube $Q_{n}$ is one of the most popular interconnection network because of its attractive properties, such as strong connectivity, small diameter, symmetry, recursive construction, relatively

\footnotetext{
*The research is supported by NSFC $(11001035,60973014,60803034)$ and SRFDP (200801081017)

${ }^{\dagger}$ Corresponding author's E-mail : yangys@dlut.edu.cn
} 
small degree, and regularity 2, 21. Naturally, the crossing number of hypercubes has attracted many researches for the past several decades (see [6, 7, $9,10,24,28]$ ).

Concerned with upper bound of crossing number of hypercube, Eggleton and Guy [7] in 1970 established a drawing of $Q_{n}$ to show

$$
\operatorname{cr}\left(Q_{n}\right) \leq \frac{5}{32} 4^{n}-\left\lfloor\frac{n^{2}+1}{2}\right\rfloor 2^{n-2} .
$$

However, a gap was found in their constructions. Erdős and Guy [8] in 1973 stated the above inequality again as a conjecture. In fact, Erdős and Guy further conjectured the equality of (1D) holds. With regard to the latest progress of this conjecture, the interested readers are referred to [9, 10, 33].

The $n$-dimensional augmented cube $A Q_{n}$ proposed by S.A. Choudum and V. Sunitha [5] in 2002 is an important variation of $Q_{n}$. The augmented cube not only retains some favorable properties of $Q_{n}$ but also processes some embedding properties that $Q_{n}$ does not [16, 17, 23]. Thus, it has drawn a great deal of attention of research $3,3,4,13,15,18,31,32$. Hence, to determine the bounds of the crossing number of the $n$-dimensional augmented cube $A Q_{n}$ would be highly interesting.

Our main result in this paper is Theorem 1.1 which gives a general upper bound of $\operatorname{cr}\left(A Q_{n}\right)$.

Theorem 1.1. For $n \geq 8$,

$$
c r\left(A Q_{n}\right)<\frac{26}{32} 4^{n}-\left(2 n^{2}+\frac{7}{2} n-6\right) 2^{n-2} .
$$

\section{Definitions and tools}

Let $\mathbb{R}$ be the set of real numbers. For any real numbers $r<s$, let $\mathbb{R}_{r}^{s}=\{t \in \mathbb{R}: r \leq t \leq s\}$. For integers $c, d \in \mathbb{Z}$ we set $[c, d]=\{t \in \mathbb{Z}: c \leq t \leq d\}$. Let $G$ be a graph, and let $A$ and $B$ be sets of vertices (not necessarily disjoint) of $G$. We denote by $E[A, B]$ the set of edges of $G$ with one end in $A$ and the other end in $B$. If $A=B$, we simply write $E(A)$ for $E[A, A]$. For any vertex subset $A \subseteq V(G)$, let $\langle A\rangle$ be the induced subgraph of $A$. Suppose that the graph $G$ is drawn in the 2-dimensional Euclidean plane $\mathbb{R} \times \mathbb{R}$. Let $u$ be a vertex of $G$. By $X_{u}$ and $Y_{u}$ we denote the $X$ and $Y$-coordinates of $u$ on $\mathbb{R} \times \mathbb{R}$.

A drawing of $G$ is said to be a good drawing, provided that no edge crosses itself, no adjacent edges cross each other, no two edges cross more than once, and no three edges cross in a point. It is well known that the crossing number of a graph is attained only in good drawings of the graph. So, we always assume that all drawings throughout this paper are good drawings. Let $A$ and $B$ be two disjoint subsets of $E(G)$. In a drawing $D$ of a graph $G$, the number of the crossings formed by an edge in $A$ and another edge in $B$ is denoted by $\nu_{D}(A, B)$, the number of the crossings that involve a pair of edges in $A$ is denoted by $\nu_{D}(A)$. For convenience, $\nu_{D}(E(G))$ is abbreviated to $\nu_{D}(G)$. Then the following statement is straightforward.

Lemma 2.1. Let $A, B, C$ be mutually disjoint subsets of $E(G)$. Then,

$$
\begin{array}{ll}
\nu_{D}(C, A \cup B) & =\nu_{D}(C, A)+\nu_{D}(C, B), \\
\nu_{D}(A \cup B) & =\nu_{D}(A)+\nu_{D}(B)+\nu_{D}(A, B) .
\end{array}
$$

The following observation will be useful for the calculations in Section 3 .

Observation 2.1. For any $m \geq 1$, let $R$ and $S$ be two non-horizontal bunches of $m$ parallel lines starting from points $(0,0),(1,0), \ldots,(m-1,0)$ respectively (see Figure 2.1), which are above the real $X$-axis. Then the number of crossings between $R$ and $S$ is $\left(\begin{array}{c}a \\ 2\end{array}\right)$.

Now we give the definition of the augmented cubes $A Q_{n}$. As with augmented hypercubes, there are many ways to describe it, one of which is as follows.

Definition 2.1. The n-dimensional augmented cube $A Q_{n}$ is defined recursively as follows: $A Q_{1}$ is a complete graph $K_{2}$ with the vertex set $\{0,1\}$. For $n \geq 2, A Q_{n}$ is obtained by taking two copies of the augmented cube $A Q_{n-1}$, denoted by $A Q_{n-1}^{0}$ and $A Q_{n-1}^{1}$, and adding $2 \times 2^{n-1}$ edges between the two as follows:

Let $V\left(A Q_{n-1}^{0}\right)=\left\{0 a_{n-1} \cdots a_{2} a_{1}: a_{i} \in\{0,1\}\right\}$ and $V\left(A Q_{n-1}^{1}\right)=\left\{1 b_{n-1} \cdots b_{2} b_{1}: b_{i} \in\{0,1\}\right\}$. A vertex $a=0 a_{n-1} \cdots a_{2} a_{1}$ of $A Q_{n-1}^{0}$ is joined to a vertex $b=1 b_{n-1} \cdots b_{2} b_{1}$ of $A Q_{n-1}^{1}$ if and only if, either $a_{i}=b_{i}$ for all $i \in[1, n-1]$, or $a_{i}=\bar{b}_{i}$ for all $i \in[1, n-1]$. 


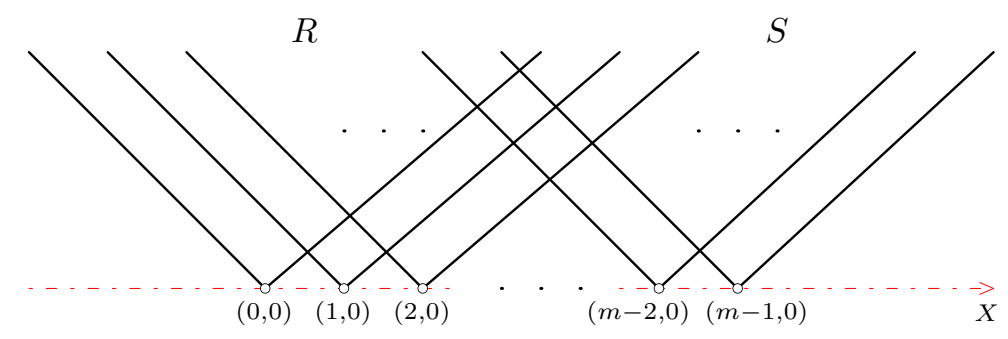

Figure 2.1: The crossings between two bunches of $m$ parallel lines $R$ and $S$

The graphs shown in Figure 2.2 are $A Q_{1}, A Q_{2}$ and $A Q_{3}$, respectively.
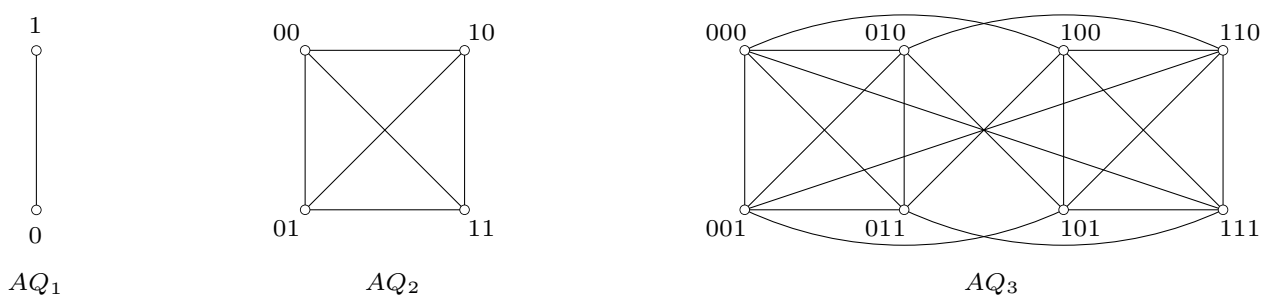

Figure 2.2: Augmented cubes $A Q_{1}, A Q_{2}$ and $A Q_{3}$

Let $a=a_{n} a_{n-1} \cdots a_{1}$ and $b=b_{n} b_{n-1} \cdots b_{1}$ be two vertices of $A Q_{n}$. We define

$$
\theta_{i}(a)=a_{i} \quad \text { for } i \in[1, n]
$$

and

$$
\operatorname{Dim}(a, b)= \begin{cases}t, & \text { if } t=1 \text { or } \theta_{t-1}(a)=\theta_{t-1}(b) \\ -t, & \text { otherwise }\end{cases}
$$

where $t$ is the largest integer $i \in[1, n]$ such that $\theta_{i}(a) \neq \theta_{i}(b)$. For convenience, let $\operatorname{Dim}(a, a)=0$. In particular, if $a b \in E\left(A Q_{n}\right)$, let $\operatorname{Dim}(a b)=\operatorname{Dim}(a, b)$ and say the edge $a b$ is of $\operatorname{Dimension} \operatorname{Dim}(a b)$. It is easy to see that

$$
\operatorname{Dim}(e) \in[-n,-2] \cup[1, n] \text { for any edge } e \in E\left(A Q_{n}\right) .
$$

For any $t \in[-n,-2] \cup[1, n]$, we define

$$
\mathscr{I}_{t}: V\left(A Q_{n}\right) \rightarrow E\left(A Q_{n}\right)
$$

to be a map such that $\mathscr{I}_{t}(a) \in E\left(A Q_{n}\right)$ is an edge incident to $a$ with

$$
\operatorname{Dim}\left(\mathscr{I}_{t}(a)\right)=t .
$$

In particular, for any vertex subset $A \subseteq V\left(A Q_{n}\right)$, let

$$
\mathscr{I}_{t}(A)=\left\{\mathscr{I}_{t}(a): a \in A\right\} .
$$

Next we shall introduce a partition of $V\left(A Q_{n}\right)$ and $E\left(A Q_{n}\right)$ for $n \geq 5$, together with a way to obtain such a partition inductively, which will be used in the drawing of $A Q_{n}$ in Section 3.

Let

$$
U^{n}=\left\{a \in V\left(A Q_{n}\right): \theta_{n-1}(a)=0\right\}
$$

and

$$
V^{n}=\left\{a \in V\left(A Q_{n}\right): \theta_{n-1}(a)=1\right\} .
$$

Hence, we have the following partition

$$
V\left(A Q_{n}\right)=U^{n} \cup V^{n}
$$


Let $\widehat{a}$ be the vertex of $A Q_{n}$ which is adjacent to $a$ with $\operatorname{Dim}(\widehat{a}, a)=-(n-2)$. We define

$$
\pi: V\left(A Q_{n}\right) \rightarrow V\left(A Q_{n+1}\right)
$$

to be a map such that

$$
\theta_{i}(\pi(a))= \begin{cases}\theta_{i}(a), & \text { if } \quad i \in[1, n-1] \\ \theta_{i-1}(a), & \text { if } \quad i \in\{n, n+1\}\end{cases}
$$

For any subset $A \subseteq V\left(A Q_{n}\right)$, we define

$$
\Omega(A)=\{\pi(a): a \in A\} \cup\{\widehat{\pi(a)}: a \in A\}
$$

and

$$
\Omega^{(m)}(A)=\underbrace{\Omega(\Omega(\cdots(\Omega}_{m}(A)) \cdots)) \quad \text { for } m \geq 1 .
$$

For convenience, let

$$
\Omega^{(0)}(A)=A
$$

It is easy to show that

$$
\Omega(\{a\}) \cap \Omega(\{b\})=\emptyset
$$

for any two distinct vertices $a, b \in V\left(A Q_{n}\right)$ with $n \geq 5$. Since $\left|U^{n}\right|=\left|V^{n}\right|=2^{n-1}$, it follows from (2), (3), (4), (5) and (6) that

$$
U^{n+1}=\Omega\left(U^{n}\right)
$$

and

$$
V^{n+1}=\Omega\left(V^{n}\right)
$$

where $n \geq 5$.

Now we define eight vertex subsets $U_{1}^{n}, U_{2}^{n}, U_{3}^{n}, U_{4}^{n}, V_{1}^{n}, V_{2}^{n}, V_{3}^{n}, V_{4}^{n}$ of $V\left(A Q_{n}\right)$ inductively for $n \geq 5$ as follows:

$$
\text { If } n=5 \text {, let }
$$

$$
\begin{aligned}
U_{1}^{5} & =\{00100,00011,00111,00000\} \\
U_{2}^{5} & =\{00101,00010,00110,00001\} \\
U_{3}^{5} & =\{10100,10011,10111,10000\} \\
U_{4}^{5} & =\{10101,10010,10110,10001\} \\
V_{1}^{5} & =\{01011,01100,01000,01111\} \\
V_{2}^{5} & =\{01010,01101,01001,01110\} \\
V_{3}^{5} & =\{11011,11100,11000,11111\} \\
V_{4}^{5} & =\{11010,11101,11001,11110\}
\end{aligned}
$$

If $n>5$, let

$$
U_{i}^{n}=\Omega\left(U_{i}^{n-1}\right)
$$

and

where $i \in\{1,2,3,4\}$.

$$
V_{i}^{n}=\Omega\left(V_{i}^{n-1}\right)
$$

By (6), we have that the above eight vertex subsets are pairwise disjoint. Moreover, by (2), (3), (41) and (5), we have that

$$
U^{n}=\bigcup_{i=1}^{4} U_{i}^{n}
$$

and

$$
V^{n}=\bigcup_{i=1}^{4} V_{i}^{n}
$$

Observe

$$
E\left(A Q_{n}\right)=E\left[U^{n}, V^{n}\right] \cup E\left(U^{n}\right) \cup E\left(V^{n}\right)
$$

By induction on $n$ and straight verifications, we can prove the following lemma which gives a partition of $E\left(A Q_{n}\right)$ and a way to obtain $E\left(A Q_{n+1}\right)$ from $E\left(A Q_{n}\right)$. 
Lemma 2.2. Let $n \geq 5$. Then,

1. $E\left[U^{n}, V^{n}\right]=\left\{e \in E\left(A Q_{n}\right): \operatorname{Dim}(e) \in\{-n,-(n-1), n-1\}\right\}$. Moreover, for any $u v \in E\left[U^{n}, V^{n}\right]$,

$$
E[\Omega(\{u\}), \Omega(\{v\})]= \begin{cases}\{\pi(u) \pi(v), \widehat{\pi(u)} \widehat{\pi(v)}, \pi(u) \widehat{\pi(v)}, \widehat{\pi(u)} \pi(v)\}, & \text { if } \operatorname{Dim}(u v)=-(n-1) ; \\ \{\pi(u) \pi(v), \widehat{\pi(u)} \widehat{\pi(v)}\}, & \text { if } \operatorname{Dim}(u v)=-n \\ \emptyset, & \text { if } \operatorname{Dim}(u v)=n-1,\end{cases}
$$

with

$$
\operatorname{Dim}(\widehat{\pi(u)} \widehat{\pi(v)})=\operatorname{Dim}(\pi(u) \pi(v))=\operatorname{Dim}(u v)-1 \text { if } \operatorname{Dim}(u v) \in\{-n,-(n-1)\}
$$

and

$$
\operatorname{Dim}(\pi(u) \widehat{\pi(v)})=\operatorname{Dim}(\widehat{\pi(u)} \pi(v))=n \text { if } \operatorname{Dim}(u v)=-(n-1) .
$$

2. $E\left(U^{n}\right) \cup E\left(V^{n}\right)=\left\{e \in E\left(A Q_{n}\right): \operatorname{Dim}(e) \in[-(n-2),-2] \cup[1, n-2] \cup\{n\}\right\}$. Moreover, for any $u v \in E\left(U^{n}\right) \cup E\left(V^{n}\right)$,

$$
E[\Omega(\{u\}), \Omega(\{v\})]=\left\{\begin{array}{lll}
\{\pi(u) \pi(v), \widehat{\pi(u)} \widehat{\pi(v)}, \pi(u) \widehat{\pi(v)}, \widehat{\pi(u)} \pi(v)\}, & \text { if } \quad \operatorname{Dim}(u v)=-(n-2) ; \\
\{\pi(u) \pi(v), \widehat{\pi(u)} \widehat{\pi(v)}\}, & \text { if } \quad \operatorname{Dim}(u v) \in[-(n-3),-2] \cup[1, n-2] \cup\{n\},
\end{array}\right.
$$

with

$$
\operatorname{Dim}(\widehat{\pi(u)} \widehat{\pi(v)})=\operatorname{Dim}(\pi(u) \pi(v))=\left\{\begin{array}{lll}
\operatorname{Dim}(u v), & \text { if } & \operatorname{Dim}(u v) \in[-(n-2),-2] \cup[1, n-2] ; \\
n+1, & \text { if } & \operatorname{Dim}(u v)=n,
\end{array}\right.
$$

and

$$
\operatorname{Dim}(\pi(u) \widehat{\pi(v)})=\operatorname{Dim}(\widehat{\pi(u)} \pi(v))=n-1 \text { if } \operatorname{Dim}(u v)=-(n-2) .
$$

3. For any two distinct vertices $u, v \in V\left(A Q_{n}\right)$ such that $u$ and $v$ are not adjacent,

$$
E[\Omega(\{u\}), \Omega(\{v\})]=\emptyset .
$$

4. $E\left(A Q_{n}\right)$ has a partition as follows:

$$
\begin{aligned}
& E\left[U_{1}^{n}, U_{2}^{n}\right] \cup E\left[U_{3}^{n}, U_{4}^{n}\right] \cup E\left[V_{1}^{n}, V_{2}^{n}\right] \cup E\left[V_{3}^{n}, V_{4}^{n}\right]=\left\{e \in E\left(A Q_{n}\right): \operatorname{Dim}(e) \in\{1,2\}\right\}, \\
& E\left[U_{1}^{n}, U_{3}^{n}\right] \cup E\left[U_{2}^{n}, U_{4}^{n}\right] \cup E\left[V_{1}^{n}, V_{3}^{n}\right] \cup E\left[V_{2}^{n}, V_{4}^{n}\right]=\left\{e \in E\left(A Q_{n}\right): \operatorname{Dim}(e)=n\right\}, \\
& E\left[U_{1}^{n}, V_{3}^{n}\right] \cup E\left[U_{2}^{n}, V_{4}^{n}\right] \cup E\left[V_{1}^{n}, U_{3}^{n}\right] \cup E\left[V_{2}^{n}, U_{4}^{n}\right]=\left\{e \in E\left(A Q_{n}\right): \operatorname{Dim}(e)=-n\right\} \text {, } \\
& \left(\cup_{i=1}^{4} E\left(U_{i}^{n}\right)\right) \cup\left(\cup_{i=1}^{4} E\left(V_{i}^{n}\right)\right)=\left\{e \in E\left(A Q_{n}\right): \operatorname{Dim}(e) \in[-(n-2),-2] \cup[3, n-2]\right\}, \\
& \cup_{i=1}^{4} E\left[U_{i}^{n}, V_{i}^{n}\right] \quad=\left\{e \in E\left(A Q_{n}\right): \operatorname{Dim}(e) \in\{-(n-1), n-1\}\right\} \text {. }
\end{aligned}
$$

In the rest of this section, we shall introduce a particular drawing of some induced subgraph of $A Q_{n}$ together with some properties of the drawing, which will be useful in Section 3.

Fix a positive integer

$$
\mathcal{N} \geq 5
$$

Take four vertices in $V\left(A Q_{\mathcal{N}}\right)$, denoted $z_{1}^{\mathcal{N}+(0)}, z_{2}^{\mathcal{N}+(0)}, z_{3}^{\mathcal{N}+(0)}, z_{4}^{\mathcal{N}+(0)}$, such that (7) and (8) hold:

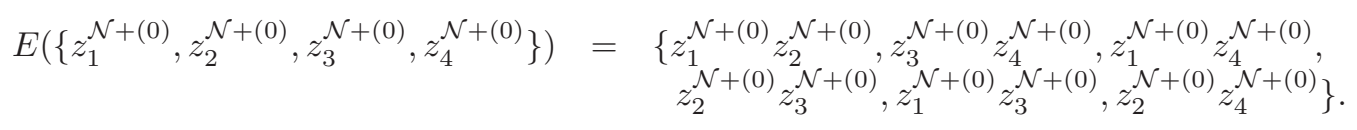

$$
\begin{array}{ll}
\operatorname{Dim}\left(z_{1}^{\mathcal{N}+(0)} z_{2}^{\mathcal{N}+(0)}\right) & =\operatorname{Dim}\left(z_{3}^{\mathcal{N}+(0)} z_{4}^{\mathcal{N}+(0)}\right)=-(\mathcal{N}-2) \\
\operatorname{Dim}\left(z_{1}^{\mathcal{N}+(0)} z_{4}^{\mathcal{N}+(0)}\right) & =\operatorname{Dim}\left(z_{2}^{\mathcal{N}+(0)} z_{3}^{\mathcal{N}+(0)}\right)=t_{1} \\
\operatorname{Dim}\left(z_{1}^{\mathcal{N}+(0)} z_{3}^{\mathcal{N}+(0)}\right) & =\operatorname{Dim}\left(z_{2}^{\mathcal{N}+(0)} z_{4}^{\mathcal{N}+(0)}\right)=t_{2}
\end{array}
$$

where

$$
\left\{t_{1}, t_{2}\right\}=\{-(\mathcal{N}-3), \mathcal{N}-2\}
$$


By (2), (3) and (8), we see that either

$$
z_{j}^{\mathcal{N}+(0)} \in U^{\mathcal{N}} \text { for all } j \in[1,4]
$$

or

$$
z_{j}^{\mathcal{N}+(0)} \in V^{\mathcal{N}} \text { for all } j \in[1,4]
$$

For $m \geq 0$, we define $\Upsilon_{(m)}^{\mathcal{N}}$ to be the drawing of the induced subgraph $\left\langle\Omega^{(m)}\left(\left\{z_{1}^{\mathcal{N}+(0)}, z_{2}^{\mathcal{N}+(0)}, z_{3}^{\mathcal{N}+(0)}, z_{4}^{\mathcal{N}+(0)}\right\}\right)\right\rangle$ of $A Q_{\mathcal{N}+m}$ such that all the vertices are drawn precisely at some axis, say the real $\mathrm{X}$-axis, and all the edges drawn to be semi-circles above or below the X-axis, and satisfies Inductive Rule A below. For convenience, we shall denote all the vertices of $\Omega^{(m)}\left(\left\{z_{1}^{\mathcal{N}+(0)}, z_{2}^{\mathcal{N}+(0)}, z_{3}^{\mathcal{N}+(0)}, z_{4}^{\mathcal{N}+(0)}\right\}\right)$ by $z_{1}^{\mathcal{N}+(m)}, z_{2}^{\mathcal{N}+(m)}, \ldots, z_{2^{m+2}}^{\mathcal{N}+(m)}$ such that

$$
X_{z_{1}^{\mathcal{N}+(m)}}<X_{z_{2}^{\mathcal{N}+(m)}}<\cdots<X_{z_{2^{m}+2}^{\mathcal{N}+(m)}}
$$

\section{Inductive Rule A.}

We consider the case when $m=0$. Draw $\left\langle\left\{z_{1}^{\mathcal{N}+(0)}, z_{2}^{\mathcal{N}+(0)}, z_{3}^{\mathcal{N}+(0)}, z_{4}^{\mathcal{N}+(0)}\right\}\right\rangle$ such that Conditions (i) and (ii) hold.

$$
X_{z_{i}^{\mathcal{N}+(0)}}=i \text { for } i \in[1,4]
$$

$$
\mathcal{O}\left(z_{1}^{\mathcal{N}+(0)} z_{2}^{\mathcal{N}+(0)}\right)=\mathcal{O}\left(z_{3}^{\mathcal{N}+(0)} z_{4}^{\mathcal{N}+(0)}\right)=-1
$$

and

$$
\mathcal{O}\left(z_{1}^{\mathcal{N}+(0)} z_{3}^{\mathcal{N}+(0)}\right)=\mathcal{O}\left(z_{2}^{\mathcal{N}+(0)} z_{4}^{\mathcal{N}+(0)}\right)=\mathcal{O}\left(z_{1}^{\mathcal{N}+(0)} z_{4}^{\mathcal{N}+(0)}\right)=\mathcal{O}\left(z_{2}^{\mathcal{N}+(0)} z_{3}^{\mathcal{N}+(0)}\right)=1
$$

where

$$
\mathcal{O}(e)= \begin{cases}1, & \text { if } e \text { is drawn above the } \mathrm{X} \text {-axis; } \\ -1, & \text { if } e \text { is drawn below the } \mathrm{X} \text {-axis, }\end{cases}
$$

for any $e \in E\left(\left\{z_{1}^{\mathcal{N}+(0)}, z_{2}^{\mathcal{N}+(0)}, z_{3}^{\mathcal{N}+(0)}, z_{4}^{\mathcal{N}+(0)}\right\}\right)$.

Now we consider the case when $m>0$.

We first arrange the vertices of $\left\langle\Omega^{(m)}\left(\left\{z_{1}^{\mathcal{N}+(0)}, z_{2}^{\mathcal{N}+(0)}, z_{3}^{\mathcal{N}+(0)}, z_{4}^{\mathcal{N}+(0)}\right\}\right)\right\rangle$. Take an arbitrary vertex $a=$ $z_{i}^{\mathcal{N}+(m-1)}$ where $i \in\left[1,2^{m+1}\right]$. We set

$$
X_{\pi(a)}=X_{a}
$$

and

$$
X_{\widehat{\pi(a)}}=X_{z_{i}^{\mathcal{N}+(m-1)}}+\frac{X_{z_{i+(-1)^{\mathcal{N}}+1}^{i-1}}-X_{z_{i}^{\mathcal{N}+(m-1)}}}{3} .
$$

It follows that

$$
\pi\left(z_{i}^{\mathcal{N}+(m-1)}\right)=z_{2 i-\frac{1+(-1)^{i-1}}{2}}^{\mathcal{N}+(m)}
$$

and

for all $i \in\left[1,2^{m+1}\right]$.

$$
\overline{\pi\left(z_{i}^{\mathcal{N}+(m-1)}\right)}=z_{2 i-\frac{1+(-1)^{i}}{2}}^{\mathcal{N}+(m)}
$$

Next we arrange the edges of $\left\langle\Omega^{(m)}\left(\left\{z_{1}^{\mathcal{N}+(0)}, z_{2}^{\mathcal{N}+(0)}, z_{3}^{\mathcal{N}+(0)}, z_{4}^{\mathcal{N}+(0)}\right\}\right)\right\rangle$. By (7), (8) and applying Conclusions 2,3 of Lemma 2.2 repeatedly, we can show that

$$
\begin{aligned}
& \left\{\operatorname{Dim}(e): e \in E\left(\Omega^{(m)}\left(\left\{z_{1}^{\mathcal{N}+(0)}, z_{2}^{\mathcal{N}+(0)}, z_{3}^{\mathcal{N}+(0)}, z_{4}^{\mathcal{N}+(0)}\right\}\right)\right)\right\} \\
= & {[-(\mathcal{N}+m-2),-(\mathcal{N}-3)] \cup[\mathcal{N}-2, \mathcal{N}+m-2] . }
\end{aligned}
$$


Combined with (9) and (10), we have

$$
\begin{aligned}
& \left\{e \in E\left(\Omega^{(m)}\left(\left\{z_{1}^{\mathcal{N}+(0)}, z_{2}^{\mathcal{N}+(0)}, z_{3}^{\mathcal{N}+(0)}, z_{4}^{\mathcal{N}+(0)}\right\}\right)\right): \operatorname{Dim}(e)=-(\mathcal{N}+m-2)\right\} \\
= & \left\{z_{2 i-1}^{\mathcal{N}+(m)} z_{2 i}^{\mathcal{N}+(m)}: i \in\left[1,2^{m+1}\right]\right\} .
\end{aligned}
$$

Draw

$$
\mathcal{O}\left(z_{2 i-1}^{\mathcal{N}+(m)} z_{2 i}^{\mathcal{N}+(m)}\right)=-\mathcal{O}\left(z_{i}^{\mathcal{N}+(m-1)} z_{i+(-1)^{i-1}}^{\mathcal{N}+(m-1)}\right) \text { for all } i \in\left[1,2^{m+1}\right]
$$

Let $z_{k}^{\mathcal{N}+(m-1)} z_{\ell}^{\mathcal{N}+(m-1)}$ be an arbitrary edge of $\left\langle\Omega^{(m-1)}\left(\left\{z_{1}^{\mathcal{N}+(0)}, z_{2}^{\mathcal{N}+(0)}, z_{3}^{\mathcal{N}+(0)}, z_{4}^{\mathcal{N}+(0)}\right\}\right)\right\rangle$, where

$$
1 \leq k<\ell \leq 2^{m+1}
$$

Suppose $\ell=k+1$ and $k \equiv 1(\bmod 2)$. By Conclusion 2 of Lemma 2.2 (9), (10) and (12), we see

$$
E\left[\Omega\left(\left\{z_{k}^{\mathcal{N}+(m-1)}\right\}\right), \Omega\left(\left\{z_{\ell}^{\mathcal{N}+(m-1)}\right\}\right)\right]=\left\{z_{2 k-1}^{\mathcal{N}+(m)} z_{2 \ell-1}^{\mathcal{N}+(m)}, z_{2 k-1}^{\mathcal{N}+(m)} z_{2 \ell}^{\mathcal{N}+(m)}, z_{2 k}^{\mathcal{N}+(m)} z_{2 \ell-1}^{\mathcal{N}+(m)}, z_{2 k}^{\mathcal{N}+(m)} z_{2 \ell}^{\mathcal{N}+(m)}\right\}
$$

and draw

$\mathcal{O}\left(z_{2 k-1}^{\mathcal{N}+(m)} z_{2 \ell-1}^{\mathcal{N}+(m)}\right)=\mathcal{O}\left(z_{2 k-1}^{\mathcal{N}+(m)} z_{2 \ell}^{\mathcal{N}+(m)}\right)=\mathcal{O}\left(z_{2 k}^{\mathcal{N}+(m)} z_{2 \ell-1}^{\mathcal{N}+(m)}\right)=\mathcal{O}\left(z_{2 k}^{\mathcal{N}+(m)} z_{2 \ell}^{\mathcal{N}+(m)}\right)=\mathcal{O}\left(z_{k}^{\mathcal{N}+(m-1)} z_{\ell}^{\mathcal{N}+(m-1)}\right)$.

Suppose $\ell=k+2$ and $k \equiv 1,2(\bmod 4)$. By Conclusion 2 of Lemma 2.2, (91), (10) and (11), we see

$$
E\left[\Omega\left(\left\{z_{k}^{\mathcal{N}+(m-1)}\right\}\right), \Omega\left(\left\{z_{\ell}^{\mathcal{N}+(m-1)}\right\}\right)\right]=\left\{\begin{array}{c}
z_{2 k-\frac{1+(-1)^{k-1}}{2}}^{\mathcal{N}+(m)} z^{\mathcal{N}+(m)} 2 \ell-\frac{1+(-1)^{\ell-1}}{2}, \\
z_{2 k-\frac{1+(-1)^{k}}{2}} z_{2 \ell-\frac{1+(-1)^{\ell}}{2}}^{\mathcal{N}+(m)}
\end{array}\right\}
$$

and draw

and

$$
\mathcal{O}\left(z_{2 k-\frac{1+(-1)^{k-1}}{2}}^{\mathcal{N}+(m)} z_{2 \ell-\frac{1+(-1)^{\ell-1}}{2}}^{\mathcal{N}+(m)}=-\mathcal{O}\left(z_{k}^{\mathcal{N}+(m-1)} z_{\ell}^{\mathcal{N}+(m-1)}\right)\right.
$$

$$
\mathcal{O}\left(z_{2 k-\frac{1+(-1)^{k}}{2}}^{\mathcal{N}+(m)} z_{2 \ell-\frac{1+(-1)^{\ell}}{2}}^{\mathcal{N}+(m)}=\mathcal{O}\left(z_{k}^{\mathcal{N}+(m-1)} z_{\ell}^{\mathcal{N}+(m-1)}\right)\right.
$$

Suppose otherwise. By Conclusion 2 of Lemma 2.2, (9), (10), (11) and (12), we see

$$
E\left[\Omega\left(\left\{z_{k}^{\mathcal{N}+(m-1)}\right\}\right), \Omega\left(\left\{z_{\ell}^{\mathcal{N}+(m-1)}\right\}\right)\right]=\left\{z_{2 k-\frac{1+(-1)^{k-1}}{2}}^{\mathcal{N}+(m)} z_{2 \ell-\frac{1+(-1)^{\ell-1}}{2}}^{\mathcal{N}+(m)}, \quad z_{2 k-\frac{1+(-1)^{k}}{2} z_{2 \ell-\frac{1+(-1)^{\ell}}{2}}^{\mathcal{N}+(m)}}^{\mathcal{N}+(m)}\right.
$$

and draw

$$
\mathcal{O}\left(z_{2 k-\frac{1+(-1)^{k-1}}{2}}^{\mathcal{N}+(m)} z_{2 \ell-\frac{1+(-1)^{\ell-1}}{2}}^{\mathcal{N}+(m)}=\mathcal{O}\left(z_{2 k-\frac{1+(-1)^{k}}{2}}^{\mathcal{N}+(m)} z_{2 \ell-\frac{1+(-1)^{\ell}}{2}}^{\mathcal{N}+(m)}=\mathcal{O}\left(z_{k}^{\mathcal{N}+(m-1)} z_{\ell}^{\mathcal{N}+(m-1)}\right)\right.\right.
$$

This completes the characterization of the drawing $\Upsilon_{(m)}^{\mathcal{N}}$.

- We say the initial positive order of the above characterized drawing $\Upsilon_{(m)}^{\mathcal{N}}$ is $\left(z_{1}^{\mathcal{N}+(0)}, z_{2}^{\mathcal{N}+(0)}, z_{3}^{\mathcal{N}+(0)}, z_{4}^{\mathcal{N}+(0)}\right)$.

By the above arguments, we see that the drawing of $\Upsilon_{(m)}^{\mathcal{N}}$ is independent of the value of $\mathcal{N}$. Therefore, for the convenience, we shall write $\Upsilon_{(m)}$ for $\Upsilon_{(m)}^{\mathcal{N}}$, and write $z_{i}^{(m)}$ for $z_{i}^{\mathcal{N}+(m)}$, and write $\mathcal{G}^{(m)}$ for the induced subgraph $\Omega^{(m)}\left(\left\{z_{1}^{(0)}, z_{2}^{(0)}, z_{3}^{(0)}, z_{4}^{(0)}\right\}\right)$ when it is unambiguous. While, in the rest of this paper, we always mean $\left\{z_{1}^{(0)}, z_{2}^{(0)}, z_{3}^{(0)}, z_{4}^{(0)}\right\} \subseteq V\left(A Q_{\mathcal{N}}\right)$ when we use $\mathcal{N}$.

To make the above notations clear, we give the drawings $\Upsilon_{(0)}, \Upsilon_{(1)}, \Upsilon_{(2)}$ and $\Upsilon_{(3)}$ as examples shown in Figure 2.3.

Before giving the properties of the drawing $\Upsilon_{(m)}$, we need to fix some notations.

Let

$$
\begin{aligned}
\mathcal{E}^{(m)} & =E\left(\mathcal{G}^{(m)}\right), \\
\mathcal{E}_{\ell}^{(m)} & =E\left(\left\{z_{i}^{(m)}: i \in\left[1,2^{m+1}\right]\right\}\right), \\
\mathcal{E}_{r}^{(m)} & =E\left(\left\{z_{i}^{(m)}: i \in\left[2^{m+1}+1,2^{m+2}\right]\right\}\right), \\
\mathcal{H}^{(m)} & =E\left[\left\{z_{i}^{(m)}: i \in\left[1,2^{m+1}\right]\right\},\left\{z_{i}^{(m)}: i \in\left[2^{m+1}+1,2^{m+2}\right]\right\}\right] .
\end{aligned}
$$




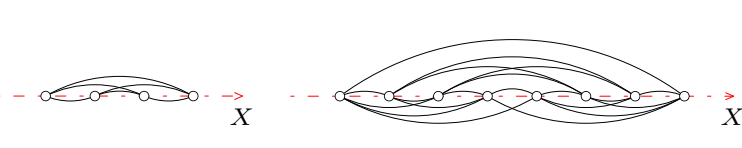

$z_{1}^{(0)} z_{2}^{(0)} z_{3}^{(0)} z_{4}^{(0)} \quad z_{1}^{(1)} z_{2}^{(1)} z_{3}^{(1)} z_{4}^{(1)} z_{5}^{(1)} z_{6}^{(1)} z_{7}^{(1)} z_{8}^{(1)}$

(1) $\Upsilon_{(0)}$

(2) $\Upsilon_{(1)}$

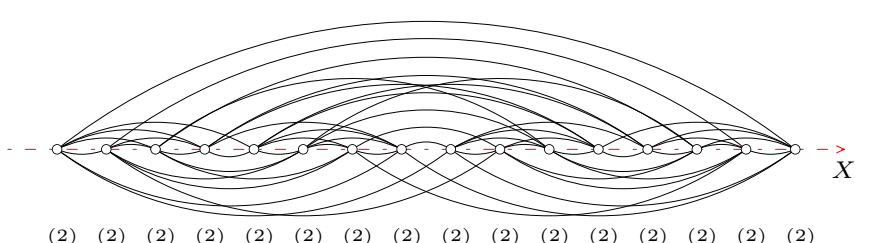

$z_{1}^{(2)} z_{2}^{(2)} z_{3}^{(2)} z_{4}^{(2)} z_{5}^{(2)} z_{6}^{(2)} z_{7}^{(2)} z_{8}^{(2)} z_{9}^{(2)} z_{10}^{(2)} z_{11}^{(2)} z_{12}^{(2)} z_{13}^{(2)} z_{14}^{(2)} z_{15}^{(2)} z_{16}^{(2)}$

(3) $\Upsilon_{(2)}$

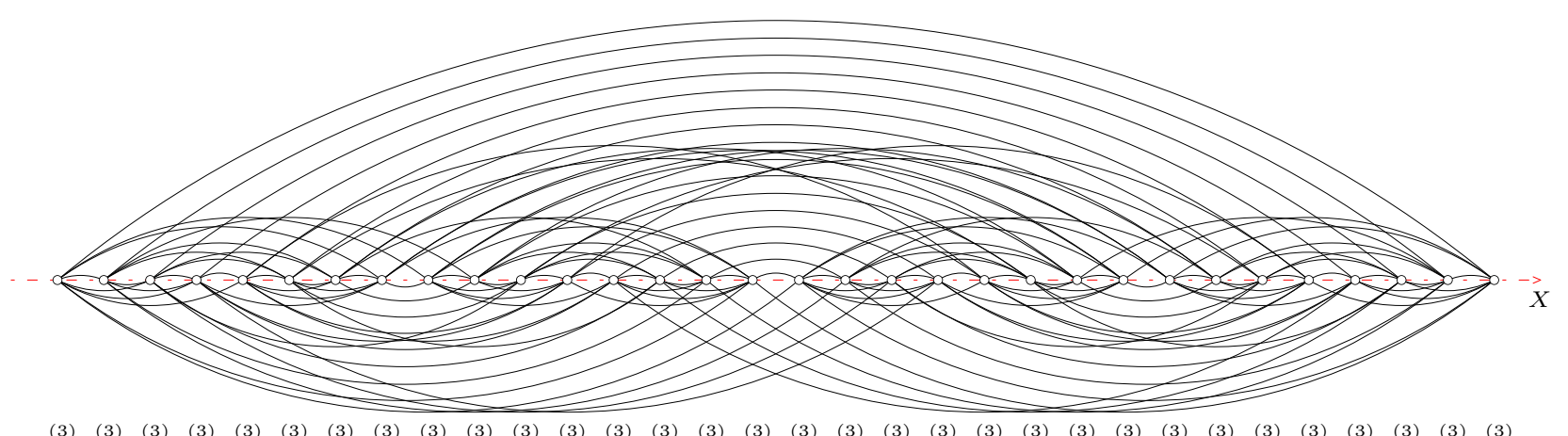

$z_{1}^{(3)} z_{2}^{(3)} z_{3}^{(3)} z_{4}^{(3)} z_{5}^{(3)} z_{6}^{(3)} z_{7}^{(3)} z_{8}^{(3)} z_{9}^{(3)} z_{10}^{(3)} z_{11}^{(3)} z_{12}^{(3)} z_{13}^{(3)} z_{14}^{(3)} z_{15}^{(3)} z_{16}^{(3)} z_{17}^{(3)} z_{18}^{(3)} z_{19}^{(3)} z_{20}^{(3)} z_{21}^{(3)} z_{22}^{(3)} z_{23}^{(3)} z_{24}^{(3)} z_{25}^{(3)} z_{26}^{(3)} z_{27}^{(3)} z_{28}^{(3)} z_{29}^{(3)} z_{30}^{(3)} z_{31}^{(3)} z_{32}^{(3)}$

(4) $\Upsilon_{(3)}$

Figure 2.3: The drawings $\Upsilon_{(0)}, \Upsilon_{(1)}, \Upsilon_{(2)}$ and $\Upsilon_{(3)}$

Let $z_{i}^{(m)} z_{j}^{(m)}$ be an edge of $\mathcal{E}^{(m)}$, where $i<j$. We say the edge $z_{i}^{(m)} z_{j}^{(m)}$ covers vertex $z_{t}^{(m)}$ for any $t \in[i+1, j-1]$. Let $F$ be an edge subset of $\mathcal{G}^{(m)}$, and let $v$ be a vertex of $\mathcal{G}^{(m)}$. For any $i \in\left[1,2^{m+2}\right]$, we define

We define

$$
\begin{aligned}
\alpha_{i}^{(m), F} & =\mid\left\{e \in F: e \text { is incident to } z_{i}^{(m)} \text { with } \mathcal{O}(e)=1\right\} \mid, \\
\beta_{i}^{(m), F} & =\mid\left\{e \in F: e \text { is incident to } z_{i}^{(m)} \text { with } \mathcal{O}(e)=-1\right\} \mid, \\
\gamma_{i}^{(m), F} & =\mid\left\{e \in F: e \text { covers } z_{i}^{(m)} \text { with } \mathcal{O}(e)=1\right\} \mid, \\
\xi_{i}^{(m), F} & =\mid\left\{e \in F: e \text { covers } z_{i}^{(m)} \text { with } \mathcal{O}(e)=-1\right\} \mid .
\end{aligned}
$$

$\mathscr{C}_{+}^{(m)}(F, v)=\mid\left\{e \in F: e\right.$ covers $v$ under the drawing $\Upsilon_{(m)}$ with $\left.\mathcal{O}(e)=1\right\} \mid$

and

$$
\mathscr{C}_{-}^{(m)}(F, v)=\mid\left\{e \in F: e \text { covers } v \text { under the drawing } \Upsilon_{(m)} \text { with } \mathcal{O}(e)=-1\right\} \mid .
$$

In particular, let

$$
\mathscr{C}_{+}^{(m)}(v)=\mathscr{C}_{+}^{(m)}\left(\mathcal{E}^{(m)}, v\right)
$$

and

Moreover, let

$$
\mathscr{C}_{-}^{(m)}(v)=\mathscr{C}_{-}^{(m)}\left(\mathcal{E}^{(m)}, v\right)
$$

$$
\mathcal{C}_{+}^{(m)}=\sum_{i \in\left[1,2^{m+2}\right]} \mathscr{C}_{+}^{(m)}\left(z_{i}^{(m)}\right)
$$

and

$$
\mathcal{C}_{-}^{(m)}=\sum_{i \in\left[1,2^{m+2}\right]} \mathscr{C}_{-}^{(m)}\left(z_{i}^{(m)}\right)
$$

Now we are ready to give some properties of the drawing $\Upsilon_{(m)}$.

By Lemma 2.2, it is not hard to derive the following two lemmas.

Lemma 2.3. Let $m \geq 0$, and let $i, j$ be two integers of $\left[1,2^{m+2}\right]$. Then,

1. Conclusions (i), (ii) and (iii) are equivalent: 
(i) $z_{i}^{(m)} z_{j}^{(m)} \in \mathcal{E}^{(m)}$;

(ii) $z_{2^{m+2}+1-i}^{(m)} z_{2^{m+2}+1-j}^{(m)} \in \mathcal{E}^{(m)}$;

(iii) $z_{i}^{(m+1)} z_{j}^{(m+1)} \in \mathcal{E}^{(m+1)}$.

\section{Furthermore,}

2. If $z_{i}^{(m)} z_{j}^{(m)} \in \mathcal{E}^{(m)}$, then $\mathcal{O}\left(z_{i}^{(m+1)} z_{j}^{(m+1)}\right)=-\mathcal{O}\left(z_{i}^{(m)} z_{j}^{(m)}\right)=-\mathcal{O}\left(z_{2^{m+2}+1-i}^{(m)} z_{2^{m+2}+1-j}^{(m)}\right)$.

Lemma 2.4. Let $m, k \geq 0$, and let $z_{i}^{(m)} z_{j}^{(m)} \in \mathcal{E}^{(m)}$ such that $i<j-2$. For any $t \in\left[1,2^{m+2}\right]$,

$$
\begin{gathered}
\sum_{v \in \Omega^{(k)}\left(\left\{z_{t}^{(m)}\right\}\right)} \mathscr{C}_{+}^{(m+k)}\left(E\left[\Omega^{(k)}\left(\left\{z_{i}^{(m)}\right\}\right), \Omega^{(k)}\left(\left\{z_{j}^{(m)}\right\}\right)\right], v\right) \\
= \begin{cases}4^{k}, & \text { if } i<t<j \text { and } \mathcal{O}\left(z_{i}^{(m)} z_{j}^{(m)}\right)=1 ; \\
2^{k-1} \cdot\left(2^{k}-1\right), & \text { if } t \in\{i, j\} \text { and } \mathcal{O}\left(z_{i}^{(m)} z_{j}^{(m)}\right)=1 ; \\
0, & \text { otherwise, }\end{cases}
\end{gathered}
$$

and

$$
\begin{gathered}
\sum_{v \in \Omega^{(k)}\left(\left\{z_{t}^{(m)}\right\}\right)} \mathscr{C}_{-}^{(m+k)}\left(E\left[\Omega^{(k)}\left(\left\{z_{i}^{(m)}\right\}\right), \Omega^{(k)}\left(\left\{z_{j}^{(m)}\right\}\right)\right], v\right) \\
= \begin{cases}4^{k}, & \text { if } i<t<j \text { and } \mathcal{O}\left(z_{i}^{(m)} z_{j}^{(m)}\right)=-1 ; \\
2^{k-1} \cdot\left(2^{k}-1\right), & \text { if } t \in\{i, j\} \text { and } \mathcal{O}\left(z_{i}^{(m)} z_{j}^{(m)}\right)=-1 ; \\
0, & \text { otherwise. }\end{cases}
\end{gathered}
$$

Lemma 2.5. Let $m>0$, and let $z_{i}^{(m)} z_{j}^{(m)} \in \mathcal{E}^{(m)}$ where $1 \leq i<j \leq 2^{m+2}$. If $j-i=2$ then $i \equiv 1,2(\bmod 4)$.

Proof. By (9) and (10), we have that $z_{i}^{(m)} \in \Omega\left(\left\{z_{\left\lceil\frac{i}{2}\right\rceil}^{(m-1)}\right\}\right)$ and $z_{j}^{(m)} \in \Omega\left(\left\{z_{\left\lceil\frac{j}{2}\right\rceil}^{(m-1)}\right\}\right)=\Omega\left(\left\{z_{\left\lceil\frac{i}{2}\right\rceil+1}^{(m-1)}\right\}\right)$, and moreover that, either $z_{i}^{(m)} \in \pi\left(\left\{z_{\left\lceil\frac{i}{2}\right\rceil}^{(m-1)}\right\}\right)$ and $z_{j}^{(m)} \in \overline{\pi\left(\left\{z_{\left\lceil\frac{i}{2}\right\rceil+1}^{(m-1)}\right\}\right)}$, or $z_{i}^{(m)} \in \overline{\pi\left(\left\{z_{\left\lceil\frac{i}{2}\right\rceil}^{(m-1)}\right\}\right)}$ and $z_{j}^{(m)} \in \pi\left(\left\{z_{\left\lceil\frac{i}{2}\right\rceil+1}^{(m-1)}\right\}\right)$. Combined with Lemma 2.2, we have that $z_{\left\lceil\frac{i}{2}\right\rceil}^{(m-1)} z_{\left\lceil\frac{i}{2}\right\rceil+1}^{(m-1)} \in \mathcal{E}^{(m-1)}$ with $\operatorname{Dim}\left(z_{\left\lceil\frac{i}{2}\right\rceil}^{(m-1)} z_{\left\lceil\frac{i}{2}\right\rceil+1}^{(m-1)}\right)=-((\mathcal{N}+m-1)-2)$. It follows from $(12)$ that $\left\lceil\frac{i}{2}\right\rceil \equiv 1(\bmod 2)$, and so $i \equiv 1,2(\bmod 4)$. The lemma follows.

By Lemma 2.5, we have that

$$
|i-j| \neq 2 \text { for any } z_{i}^{(m)} z_{j}^{(m)} \in \mathcal{H}^{(m)} \text { with } m>0 .
$$

Let $m \geq 1$. We define

$$
\mathcal{I}_{t}^{(m)}=\left[(t-1) \cdot 2^{m-1}+1, t \cdot 2^{m-1}\right] \text { for } t \in[1,8] .
$$

By (13) and Lemma 2.2, we can derive that

$$
\alpha_{i}^{(m), \mathcal{H}^{(m)}}= \begin{cases}1, & i \in \mathcal{I}_{1}^{(m)} \cup \mathcal{I}_{4}^{(m)} \cup \mathcal{I}_{5}^{(m)} \cup \mathcal{I}_{8}^{(m)} \\ 2, & i \in \mathcal{I}_{2}^{(m)} \cup \mathcal{I}_{3}^{(m)} \cup \mathcal{I}_{6}^{(m)} \cup \mathcal{I}_{7}^{(m)}\end{cases}
$$

and

$$
\beta_{i}^{(m), \mathcal{H}^{(m)}}= \begin{cases}1, & i \in \mathcal{I}_{1}^{(m)} \cup \mathcal{I}_{4}^{(m)} \cup \mathcal{I}_{5}^{(m)} \cup \mathcal{I}_{8}^{(m)} \\ 0, & i \in \mathcal{I}_{2}^{(m)} \cup \mathcal{I}_{3}^{(m)} \cup \mathcal{I}_{6}^{(m)} \cup \mathcal{I}_{7}^{(m)}\end{cases}
$$

for all $m \geq 1$.

Lemma 2.6. For $m \geq 0$,

$$
\mathcal{C}_{+}^{(m)}=\frac{7}{3} \cdot 4^{m+1}-\left(2 m+\frac{16}{3}+\frac{7 \cdot\left(1+(-1)^{m+1}\right)}{6}\right) \cdot 2^{m}
$$

and

$$
\mathcal{C}_{-}^{(m)}=\frac{5}{3} \cdot 4^{m+1}-\left(2 m+\frac{13}{3}+\frac{7 \cdot\left(1+(-1)^{m}\right)}{6}\right) \cdot 2^{m}
$$


Proof. If $m=0,1$, the lemma follows from trivial verifications (see Figure $2.3(1)-(2)$ for $\Upsilon_{(0)}$ and $\left.\Upsilon_{(1)}\right)$. Now we consider the case when

$$
m>1
$$

By (15), (16) and trivial verifications, we have the following

\begin{tabular}{|c|c|c|c|c|}
\hline$j$ & 1 & 2 & 3 & 4 \\
\hline$\alpha_{j}^{(1), \mathcal{H}^{(1)}}$ & 1 & 2 & 2 & 1 \\
\hline$\beta_{j}^{(1), \mathcal{H}^{(1)}}$ & 1 & 0 & 0 & 1 \\
\hline$\gamma_{j}^{(1), \mathcal{H}^{(1)}}$ & 0 & 1 & 3 & 5 \\
\hline$\xi_{j}^{(1), \mathcal{H}^{(1)}}$ & 0 & 1 & 1 & 1 \\
\hline
\end{tabular}

Table 2.1: The values of $\alpha_{j}^{(1), \mathcal{H}^{(1)}}, \beta_{j}^{(1), \mathcal{H}^{(1)}}, \gamma_{j}^{(1), \mathcal{H}^{(1)}}$ and $\xi_{j}^{(1), \mathcal{H}^{(1)}}$ for $j=1,2,3,4$

Combined (13), Table 2.1, Lemma 2.2, Lemma 2.3 and Lemma 2.4, we have that

$$
\begin{aligned}
\mathcal{C}_{+}^{(m)} & =\sum_{i \in\left[1,2^{m+2}\right]} \mathscr{C}_{+}^{(m)}\left(z_{i}^{(m)}\right) \\
& =2 \cdot\left(\sum_{i \in\left[1,2^{m+1}\right]} \mathscr{C}_{+}^{(m)}\left(z_{i}^{(m)}\right)\right) \\
& =2 \cdot\left(\sum_{i \in\left[1,2^{m+1}\right]} \mathscr{C}_{+}^{(m)}\left(\mathcal{E}_{\ell}^{(m)}, z_{i}^{(m)}\right)+\sum_{i \in\left[1,2^{m+1}\right]} \mathscr{C}_{+}^{(m)}\left(\mathcal{H}^{(m)}, z_{i}^{(m)}\right)\right) \\
& =2 \cdot\left(\sum_{i \in\left[1,2^{m+1}\right]} \mathscr{C}_{-}^{(m-1)}\left(\mathcal{E}^{(m-1)}, z_{i}^{(m-1)}\right)+\sum_{i \in\left[1,2^{m+1}\right]} \mathscr{C}_{+}^{(m)}\left(\mathcal{H}^{(m)}, z_{i}^{(m)}\right)\right) \\
& =2 \cdot\left(\mathcal{C}_{-}^{(m-1)}+\sum_{i \in\left[1,2^{m+1}\right]} \mathscr{C}_{+}^{(m)}\left(\mathcal{H}^{(m)}, z_{i}^{(m)}\right)\right) \\
& =2 \cdot\left(\mathcal{C}_{-}^{(m-1)}+4^{m-1} \cdot \sum_{j=1}^{4} \gamma_{j}^{(1), \mathcal{H}^{(1)}}+2^{m-2} \cdot\left(2^{m-1}-1\right) \cdot \sum_{j=1}^{4} \alpha_{j}^{(1), \mathcal{H}^{(1)}}\right) \\
& =2 \cdot \mathcal{C}_{-}^{(m-1)}+\left(6 \cdot 4^{m}-3 \cdot 2^{m}\right)
\end{aligned}
$$

and

$$
\begin{aligned}
\mathcal{C}_{-}^{(m)} & =\sum_{i \in\left[1,2^{m+2}\right]} \mathscr{C}_{-}^{(m)}\left(z_{i}^{(m)}\right) \\
& =2 \cdot\left(\sum_{i \in\left[1,2^{m+1}\right]} \mathscr{C}_{-}^{(m)}\left(z_{i}^{(m)}\right)\right) \\
& =2 \cdot\left(\sum_{i \in\left[1,2^{m+1}\right]} \mathscr{C}_{-}^{(m)}\left(\mathcal{E}_{\ell}^{(m)}, z_{i}^{(m)}\right)+\sum_{i \in\left[1,2^{m+1}\right]} \mathscr{C}_{-}^{(m)}\left(\mathcal{H}^{(m)}, z_{i}^{(m)}\right)\right) \\
& =2 \cdot\left(\sum_{i \in\left[1,2^{m+1}\right]} \mathscr{C}_{+}^{(m-1)}\left(\mathcal{E}^{(m-1)}, z_{i}^{(m-1)}\right)+\sum_{i \in\left[1,2^{m+1}\right]} \mathscr{C}_{-}^{(m)}\left(\mathcal{H}^{(m)}, z_{i}^{(m)}\right)\right) \\
& =2 \cdot\left(\mathcal{C}_{+}^{(m-1)}+\sum_{i \in\left[1,2^{m+1}\right]} \mathscr{C}_{-}^{(m)}\left(\mathcal{H}^{(m)}, z_{i}^{(m)}\right)\right) \\
& =2 \cdot\left(\mathcal{C}_{+}^{(m-1)}+4^{m-1} \cdot \sum_{j=1}^{4} \xi_{j}^{(1), \mathcal{H}^{(1)}}+2^{m-2} \cdot\left(2^{m-1}-1\right) \cdot \sum_{j=1}^{4} \beta_{j}^{(1), \mathcal{H}^{(1)}}\right) \\
& =2 \cdot \mathcal{C}_{+}^{(m-1)}+\left(2 \cdot 4^{m}-2^{m}\right) .
\end{aligned}
$$

Then the lemma follows from (17) and (18). 
Lemma 2.7. The following three conclusions hold.

(i) For $m=1$,

$$
\sum_{i \in \mathcal{I}_{t}^{(m)}} \mathscr{C}_{+}^{(m)}\left(\mathcal{E}^{(m)}, z_{i}^{(m)}\right)= \begin{cases}0, & \text { if } t \in\{1,8\} \\ 1, & \text { if } t \in\{2,7\} \\ 3, & \text { if } t \in\{3,6\} \\ 5, & \text { if } t \in\{4,5\}\end{cases}
$$

and

$$
\sum_{i \in \mathcal{I}_{t}^{(m)}} \mathscr{C}_{-}^{(m)}\left(\mathcal{E}^{(m)}, z_{i}^{(m)}\right)= \begin{cases}0, & \text { if } t \in\{1,8\} \\ 3, & \text { if } t \in\{2,7\} \\ 3, & \text { if } t \in\{3,6\} \\ 1, & \text { if } t \in\{4,5\}\end{cases}
$$

(ii) For $m=2$,

$$
\sum_{i \in \mathcal{I}_{t}^{(m)}} \mathscr{C}_{+}^{(m)}\left(\mathcal{E}^{(m)}, z_{i}^{(m)}\right)= \begin{cases}4, & \text { if } t \in\{1,8\} \\ 10, & \text { if } t \in\{2,7\} \\ 18, & \text { if } t \in\{3,6\} \\ 24, & \text { if } t \in\{4,5\}\end{cases}
$$

and

$$
\sum_{i \in \mathcal{I}_{t}^{(m)}} \mathscr{C}_{-}^{(m)}\left(\mathcal{E}^{(m)}, z_{i}^{(m)}\right)=\left\{\begin{array}{lll}
2, & \text { if } t \in\{1,8\} \\
12, & \text { if } t \in\{2,7\} \\
12, & \text { if } t \in\{3,6\} \\
6, & \text { if } t \in\{4,5\}
\end{array}\right.
$$

(iii) For $m \geq 3$,

$$
\sum_{i \in \mathcal{I}_{t}^{(m)}} \mathscr{C}_{+}^{(m)}\left(\mathcal{E}^{(m)}, z_{i}^{(m)}\right)=\left\{\begin{array}{lll}
\frac{98}{3} \cdot 4^{m-3}-\left(2 m+\frac{13}{3}+\frac{7 \cdot\left(1+(-1)^{m+1}\right)}{6}\right) \cdot 2^{m-3}, & \text { if } t \in\{1,8\} ; \\
\frac{182}{3} \cdot 4^{m-3}-\left(2 m+\frac{19}{3}+\frac{7 \cdot\left(1+(-1)^{m+1}\right)}{6}\right) \cdot 2^{m-3}, & \text { if } t \in\{2,7\} ; \\
\frac{278}{3} \cdot 4^{m-3}-\left(2 m+\frac{19}{3}+\frac{7 \cdot\left(1+(-1)^{m+1}\right)}{6}\right) \cdot 2^{m-3}, & \text { if } t \in\{3,6\} ; \\
\frac{338}{3} \cdot 4^{m-3}-\left(2 m+\frac{13}{3}+\frac{7 \cdot\left(1+(-1)^{m+1}\right)}{6}\right) \cdot 2^{m-3}, & \text { if } t \in\{4,5\},
\end{array}\right.
$$

and

$$
\sum_{i \in \mathcal{I}_{t}^{(m)}} \mathscr{C}_{-}^{(m)}\left(\mathcal{E}^{(m)}, z_{i}^{(m)}\right)=\left\{\begin{array}{lll}
\frac{94}{3} \cdot 4^{m-3}-\left(2 m+\frac{16}{3}+\frac{7 \cdot\left(1+(-1)^{m}\right)}{6}\right) \cdot 2^{m-3}, & \text { if } t \in\{1,8\} \\
\frac{202}{3} \cdot 4^{m-3}-\left(2 m+\frac{10}{3}+\frac{7 \cdot\left(1+(-1)^{m}\right)}{6}\right) \cdot 2^{m-3}, & \text { if } t \in\{2,7\} \\
\frac{202}{3} \cdot 4^{m-3}-\left(2 m+\frac{10}{3}+\frac{7 \cdot\left(1+(-1)^{m}\right)}{6}\right) \cdot 2^{m-3}, & \text { if } t \in\{3,6\} \\
\frac{142}{3} \cdot 4^{m-3}-\left(2 m+\frac{16}{3}+\frac{7 \cdot\left(1+(-1)^{m}\right)}{6}\right) \cdot 2^{m-3}, & \text { if } t \in\{4,5\}
\end{array}\right.
$$

Proof. (i) and (ii) follows from straight verifications (see Figure 2.3). Now we let $m \geq 3$ and prove (iii).

For $t \in[1,8]$, let

$$
\begin{gathered}
\mathcal{E}_{t}^{(m)}=E\left(\left\{z_{i}^{(m)}: i \in \mathcal{I}_{t}^{(m)}\right\}\right), \\
\overline{\mathcal{E}_{t}^{(m)}}=\mathcal{E}^{(m)} \backslash \mathcal{E}_{t}^{(m)} .
\end{gathered}
$$

Let

$$
\mathcal{K}^{(m)}=\mathcal{E}^{(m)} \backslash \cup_{i=1}^{8} \mathcal{E}_{i}^{(m)} .
$$

By (14) and Lemma 2.5] we have that

$$
|i-j| \neq 2 \text { for any } z_{i}^{(m)} z_{j}^{(m)} \in \mathcal{K}^{(m)} \text { with } m \geq 3 .
$$


Combined (19), Lemma 2.2 Lemma 2.3. Lemma 2.4 and Lemma 2.6, we have that for any $t \in[1,8]$,

$$
\begin{aligned}
& \sum_{i \in \mathcal{I}_{t}^{(m)}} \mathscr{C}_{+}^{(m)}\left(\mathcal{E}^{(m)}, z_{i}^{(m)}\right) \\
= & \sum_{i \in \mathcal{I}_{t}^{(m)}} \mathscr{C}_{+}^{(m)}\left(\mathcal{E}_{t}^{(m)}, z_{i}^{(m)}\right)+\sum_{i \in \mathcal{I}_{t}^{(m)}} \mathscr{C}_{+}^{(m)}\left(\overline{\mathcal{E}_{t}^{(m)}}, z_{i}^{(m)}\right) \\
= & \sum_{i \in \mathcal{I}_{t}^{(m)}} \mathscr{C}_{+}^{(m)}\left(\mathcal{E}_{t}^{(m)}, z_{i}^{(m)}\right)+\sum_{i \in \mathcal{I}_{t}^{(m)}} \mathscr{C}_{+}^{(m)}\left(\mathcal{K}^{(m)}, z_{i}^{(m)}\right) \\
= & \mathcal{C}_{-}^{(m-3)}+\sum_{i \in \mathcal{I}_{t}^{(m)}} \mathscr{C}_{+}^{(m)}\left(\mathcal{K}^{(m)}, z_{i}^{(m)}\right) \\
= & \mathcal{C}_{-}^{(m-3)}+\left(4^{m-3} \cdot \sum_{j=4(t-1)+1}^{4 t} \gamma_{j}^{(3), \mathcal{K}^{(3)}}+2^{m-4} \cdot\left(2^{m-3}-1\right) \cdot \sum_{j=4(t-1)+1}^{4 t} \alpha_{j}^{(3), \mathcal{K}^{(3)}}\right)
\end{aligned}
$$

and similarly,

$$
\sum_{i \in \mathcal{I}_{t}^{(m)}} \mathscr{C}_{-}^{(m)}\left(\mathcal{E}^{(m)}, z_{i}^{(m)}\right)=\mathcal{C}_{+}^{(m-3)}+\left(4^{m-3} \cdot \sum_{j=4(t-1)+1}^{4 t} \xi_{j}^{(3), \mathcal{K}^{(3)}}+2^{m-4} \cdot\left(2^{m-3}-1\right) \cdot \sum_{j=4(t-1)+1}^{4 t} \beta_{j}^{(3), \mathcal{K}^{(3)}}\right) .
$$

It is easy to get the values in Table 2.2 .

\begin{tabular}{|c|c|c|c|c|c|c|c|c|c|c|c|c|c|c|c|c|}
\hline$j$ & 1 & 2 & 3 & 4 & 5 & 6 & 7 & 8 & 9 & 10 & 11 & 12 & 13 & 14 & 15 & 16 \\
\hline$\alpha_{j}^{(3), \mathcal{K}^{(3)}}$ & 3 & 4 & 3 & 2 & 3 & 4 & 5 & 4 & 4 & 5 & 4 & 3 & 2 & 3 & 4 & 3 \\
\hline$\beta_{j}^{(3), \mathcal{K}^{(3)}}$ & 3 & 2 & 3 & 4 & 3 & 2 & 1 & 2 & 2 & 1 & 2 & 3 & 4 & 3 & 2 & 3 \\
\hline$\gamma_{j}^{(3), \mathcal{K}^{(3)}}$ & 0 & 3 & 7 & 10 & 11 & 11 & 11 & 13 & 15 & 17 & 21 & 25 & 27 & 26 & 24 & 23 \\
\hline$\xi_{j}^{(3), \mathcal{K}^{(3)}}$ & 0 & 3 & 5 & 8 & 11 & 13 & 15 & 15 & 15 & 15 & 13 & 11 & 9 & 8 & 8 & 7 \\
\hline
\end{tabular}

Table 2.2: The values of $\alpha_{j}^{(3), \mathcal{K}^{(3)}}, \beta_{j}^{(3), \mathcal{K}^{(3)}}, \gamma_{j}^{(3), \mathcal{K}^{(3)}}$ and $\xi_{j}^{(3), \mathcal{K}^{(3)}}$ for $j \in[1,16]$

Then Conclusion (iii) follows from (20), (21), Table 2.2 and Lemma 2.6 readily.

In the following lemma, we shall count the crossings of $\Upsilon_{(m)}$ for all $m \geq 1$.

Lemma 2.8. For $m \geq 1, \nu_{\Upsilon_{(m)}}\left(\mathcal{H}^{(m)}\right)=6 \cdot 4^{m-1}-2^{m+1}$.

Proof. By (9), (10), (13) and Lemma 2.2, we have the following

Claim A. Let $z_{i}^{(m)} z_{j}^{(m)}$ and $z_{i}^{(m)} z_{k}^{(m)}$ be two distinct edges of $\mathcal{H}^{(m)}$ incident to $z_{i}^{(m)}$. Then

$$
\nu_{\Upsilon_{(m+1)}}\left(E\left[\Omega\left(\left\{z_{i}^{(m)}\right\}\right), \Omega\left(\left\{z_{j}^{(m)}\right\}\right)\right], E\left[\Omega\left(\left\{z_{i}^{(m)}\right\}\right), \Omega\left(\left\{z_{k}^{(m)}\right\}\right)\right]\right)=1 .
$$

Now we show

Claim B. Let $z_{i}^{(m)} z_{j}^{(m)}$ be an arbitrary edge of $\mathcal{H}^{(m)}$. Then

$$
\nu_{\Upsilon_{(m+1)}}\left(E\left[\Omega\left(\left\{z_{i}^{(m)}\right\}\right), \Omega\left(\left\{z_{j}^{(m)}\right\}\right)\right]\right)=\left\{\begin{array}{lll}
1, & \text { if } i \equiv j & (\bmod 2) ; \\
0, & \text { if } i \neq j j & (\bmod 2) .
\end{array}\right.
$$

Proof of Claim B. Say $i<j$. By (91), (10), (13) and Lemma 2.2, we have that

$$
\begin{aligned}
& E\left[\Omega\left(\left\{z_{i}^{(m)}\right\}\right), \Omega\left(\left\{z_{j}^{(m)}\right\}\right)\right] \\
= & \left\{\pi\left(z_{i}^{(m)}\right) \pi\left(z_{j}^{(m)}\right), \widehat{\left.\pi\left(z_{i}^{(m)}\right) \pi\left(z_{j}^{(m)}\right)\right\}}\right. \\
= & \left\{z_{2 i-\frac{1+(-1)^{i-1}}{2}}^{(m+1)} z_{2 j-\frac{1+(-1)^{j-1}}{2}}^{(m+1)} z_{2 i-\frac{1+(-1)^{i}}{2}}^{(m+1)} z_{2 j-\frac{1+(-1)^{j}}{2}}^{(m+1)}\right.
\end{aligned}
$$


Also, since $\mathcal{O}\left(z_{2 i-\frac{1+(-1)^{i-1}}{2}}^{(m+1)} z_{2 j-\frac{1+(-1)^{j-1}}{2}}^{(m+1)}\right)=\mathcal{O}\left(z_{2 i-\frac{1+(-1)^{i}}{2}}^{(m+1)} z_{2 j-\frac{1+(-1)^{j}}{2}}^{(m+1)}=\mathcal{O}\left(z_{i}^{(m)} z_{j}^{(m)}\right)\right.$, we have Claim B proved.

In a similar argument as Claim B, by induction on $m$, we have

$$
\left|\left\{z_{i}^{(m)} z_{j}^{(m)} \in \mathcal{H}^{(m)}: i \equiv j \quad(\bmod 2)\right\}\right|=2^{m+1} .
$$

Now we proceed with the proof of the lemma.

If $m=1$, the lemma holds trivially (see Figure 2.3). Suppose $m>1$. By (13), (15), (16), (22), Claim A, Claim B and Lemma 2.2, we have that

$$
\begin{aligned}
& \nu_{\Upsilon_{(m)}}\left(\mathcal{H}^{(m)}\right) \\
& =4 \cdot \nu_{\Upsilon_{(m-1)}}\left(\mathcal{H}^{(m-1)}\right)+\left|\left\{z_{i}^{(m-1)} z_{j}^{(m-1)} \in \mathcal{H}^{(m-1)}: i \equiv j \quad(\bmod 2)\right\}\right| \\
& +\sum_{i=1}^{2^{m+1}}\left(\begin{array}{c}
\alpha_{i}^{(m-1), \mathcal{H}^{(m-1)}} \\
2
\end{array}\right)+\sum_{i=1}^{2^{m+1}}\left(\begin{array}{c}
\beta_{i}^{(m-1), \mathcal{H}^{(m-1)}} \\
2
\end{array}\right) \\
& =4 \cdot \nu \Upsilon_{(m-1)}\left(\mathcal{H}^{(m-1)}\right)+2^{m} \\
& +\sum_{i \in \mathcal{I}_{1}^{(m-1)} \cup \mathcal{I}_{4}^{(m-1)} \cup \mathcal{I}_{5}^{(m-1)} \cup \mathcal{I}_{8}^{(m-1)}}\left(\begin{array}{c}
\alpha_{i}^{(m-1), \mathcal{H}}(m-1) \\
2
\end{array}\right)+\sum_{i \in \mathcal{I}_{2}^{(m-1)} \cup \mathcal{I}_{3}^{(m-1)} \cup \mathcal{I}_{6}^{(m-1)} \cup \mathcal{I}_{7}^{(m-1)}}\left(\begin{array}{c}
\alpha_{i}^{(m-1), \mathcal{H}^{(m-1)}} \\
2
\end{array}\right) \\
& +\sum_{i \in \mathcal{I}_{1}^{(m-1)} \cup \mathcal{I}_{4}^{(m-1)} \cup \mathcal{I}_{5}^{(m-1)} \cup \mathcal{I}_{8}^{(m-1)}}\left(\begin{array}{c}
\beta_{i}^{(m-1), \mathcal{H}}(m-1) \\
2
\end{array}\right)+\sum_{i \in \mathcal{I}_{2}^{(m-1)} \cup \mathcal{I}_{3}^{(m-1)} \cup \mathcal{I}_{6}^{(m-1)} \cup \mathcal{I}_{7}^{(m-1)}}\left(\beta_{i}^{(m-1), \mathcal{H}^{(m-1)}}\right) \\
& =4 \cdot \nu \Upsilon_{(m-1)}\left(\mathcal{H}^{(m-1)}\right)+2^{m} \\
& +\sum_{i \in \mathcal{I}_{1}^{(m-1)} \cup \mathcal{I}_{4}^{(m-1)} \cup \mathcal{I}_{5}^{(m-1)} \cup \mathcal{I}_{8}^{(m-1)}}\left(\begin{array}{l}
1 \\
2
\end{array}\right)+\sum_{i \in \mathcal{I}_{2}^{(m-1)} \cup \mathcal{I}_{3}^{(m-1)} \cup \mathcal{I}_{6}^{(m-1)} \cup \mathcal{I}_{7}^{(m-1)}}\left(\begin{array}{l}
2 \\
2
\end{array}\right) \\
& +\sum_{i \in \mathcal{I}_{1}^{(m-1)} \cup \mathcal{I}_{4}^{(m-1)} \cup \mathcal{I}_{5}^{(m-1)} \cup \mathcal{I}_{8}^{(m-1)}}\left(\begin{array}{l}
1 \\
2
\end{array}\right)+\sum_{i \in \mathcal{I}_{2}^{(m-1)} \cup \mathcal{I}_{3}^{(m-1)} \cup \mathcal{I}_{6}^{(m-1)} \cup \mathcal{I}_{7}^{(m-1)}}\left(\begin{array}{l}
0 \\
2
\end{array}\right) \\
& =4 \cdot \nu_{\Upsilon_{(m-1)}}\left(\mathcal{H}^{(m-1)}\right)+2^{m}+2^{m} \\
& =4 \cdot \nu_{\Upsilon_{(m-1)}}\left(\mathcal{H}^{(m-1)}\right)+2^{m+1} \text {, }
\end{aligned}
$$

and thus, the lemma follows immediately.

\section{Lemma 2.9.}

$$
\nu_{\Upsilon_{(m)}}\left(\mathcal{H}^{(m)}, \mathcal{E}_{\ell}^{(m)}\right)= \begin{cases}24, & \text { if } m=2 \\ 128, & \text { if } m=3 \\ \frac{158}{3} \cdot 4^{m-2}-\left(8 m+\frac{38}{3}+\frac{7 \cdot\left(1+(-1)^{m-1}\right)}{3}\right) \cdot 2^{m-2}, & \text { if } m \geq 4\end{cases}
$$


Proof. By (15), (16) and Lemma 2.3, we have that, for all $m \geq 2$,

$$
\begin{aligned}
& \nu_{\Upsilon_{(m)}}\left(\mathcal{H}^{(m)}, \mathcal{E}_{\ell}^{(m)}\right) \\
& =\sum_{i \in \bigcup_{t=1}^{4} \mathcal{I}_{t}^{(m)}} \alpha_{i}^{(m), \mathcal{H}^{(m)}} \cdot \mathscr{C}_{+}^{(m)}\left(\mathcal{E}_{\ell}^{(m)}, z_{i}^{(m)}\right)+\sum_{i \in \bigcup_{t=1}^{4} \mathcal{I}_{t}^{(m)}} \beta_{i}^{(m), \mathcal{H}^{(m)}} \cdot \mathscr{C}_{-}^{(m)}\left(\mathcal{E}_{\ell}^{(m)}, z_{i}^{(m)}\right) \\
& =\sum_{i \in \mathcal{I}_{1}^{(m)}} \alpha_{i}^{(m), \mathcal{H}^{(m)}} \cdot \mathscr{C}_{+}^{(m)}\left(\mathcal{E}_{\ell}^{(m)}, z_{i}^{(m)}\right)+\sum_{i \in \mathcal{I}_{2}^{(m)}} \alpha_{i}^{(m), \mathcal{H}^{(m)}} \cdot \mathscr{C}_{+}^{(m)}\left(\mathcal{E}_{\ell}^{(m)}, z_{i}^{(m)}\right) \\
& +\sum_{i \in \mathcal{I}_{3}^{(m)}} \alpha_{i}^{(m), \mathcal{H}^{(m)}} \cdot \mathscr{C}_{+}^{(m)}\left(\mathcal{E}_{\ell}^{(m)}, z_{i}^{(m)}\right)+\sum_{i \in \mathcal{I}_{4}^{(m)}} \alpha_{i}^{(m), \mathcal{H}^{(m)}} \cdot \mathscr{C}_{+}^{(m)}\left(\mathcal{E}_{\ell}^{(m)}, z_{i}^{(m)}\right) \\
& +\sum_{i \in \mathcal{I}_{1}^{(m)}} \beta_{i}^{(m), \mathcal{H}^{(m)}} \cdot \mathscr{C}_{-}^{(m)}\left(\mathcal{E}_{\ell}^{(m)}, z_{i}^{(m)}\right)+\sum_{i \in \mathcal{I}_{2}^{(m)}} \beta_{i}^{(m), \mathcal{H}^{(m)}} \cdot \mathscr{C}_{-}^{(m)}\left(\mathcal{E}_{\ell}^{(m)}, z_{i}^{(m)}\right) \\
& +\sum_{i \in \mathcal{I}_{3}^{(m)}} \beta_{i}^{(m), \mathcal{H}^{(m)}} \cdot \mathscr{C}_{-}^{(m)}\left(\mathcal{E}_{\ell}^{(m)}, z_{i}^{(m)}\right)+\sum_{i \in \mathcal{I}_{4}^{(m)}} \beta_{i}^{(m), \mathcal{H}^{(m)}} \cdot \mathscr{C}_{-}^{(m)}\left(\mathcal{E}_{\ell}^{(m)}, z_{i}^{(m)}\right) \\
& =\sum_{i \in \mathcal{I}_{1}^{(m)}} \mathscr{C}_{+}^{(m)}\left(\mathcal{E}_{\ell}^{(m)}, z_{i}^{(m)}\right)+2 \cdot \sum_{i \in \mathcal{I}_{2}^{(m)}} \mathscr{C}_{+}^{(m)}\left(\mathcal{E}_{\ell}^{(m)}, z_{i}^{(m)}\right) \\
& +2 \cdot \sum_{i \in \mathcal{I}_{3}^{(m)}} \mathscr{C}_{+}^{(m)}\left(\mathcal{E}_{\ell}^{(m)}, z_{i}^{(m)}\right)+\sum_{i \in \mathcal{I}_{4}^{(m)}} \mathscr{C}_{+}^{(m)}\left(\mathcal{E}_{\ell}^{(m)}, z_{i}^{(m)}\right) \\
& +\sum_{i \in \mathcal{I}_{1}^{(m)}} \mathscr{C}_{-}^{(m)}\left(\mathcal{E}_{\ell}^{(m)}, z_{i}^{(m)}\right)+\sum_{i \in \mathcal{I}_{4}^{(m)}} \mathscr{C}_{-}^{(m)}\left(\mathcal{E}_{\ell}^{(m)}, z_{i}^{(m)}\right) \\
& =\sum_{i \in \mathcal{I}_{1}^{(m-1)} \cup \mathcal{I}_{2}^{(m-1)}} \mathscr{C}_{-}^{(m-1)}\left(\mathcal{E}^{(m-1)}, z_{i}^{(m-1)}\right)+2 \cdot \sum_{i \in \mathcal{I}_{3}^{(m-1)} \cup \mathcal{I}_{4}^{(m-1)}} \mathscr{C}_{-}^{(m-1)}\left(\mathcal{E}^{(m-1)}, z_{i}^{(m-1)}\right) \\
& +2 \cdot \sum_{i \in \mathcal{I}_{5}^{(m-1)} \cup \mathcal{I}_{6}^{(m-1)}} \mathscr{C}_{-}^{(m-1)}\left(\mathcal{E}^{(m-1)}, z_{i}^{(m-1)}\right)+\sum_{i \in \mathcal{I}_{7}^{(m-1)} \cup \mathcal{I}_{8}^{(m-1)}} \mathscr{C}_{-}^{(m-1)}\left(\mathcal{E}^{(m-1)}, z_{i}^{(m-1)}\right) \\
& +\sum_{i \in \mathcal{I}_{1}^{(m-1)} \cup \mathcal{I}_{2}^{(m-1)}} \mathscr{C}_{+}^{(m-1)}\left(\mathcal{E}^{(m-1)}, z_{i}^{(m-1)}\right)+\sum_{i \in \mathcal{I}_{7}^{(m-1)} \cup \mathcal{I}_{8}^{(m-1)}} \mathscr{C}_{+}^{(m-1)}\left(\mathcal{E}^{(m-1)}, z_{i}^{(m-1)}\right) \\
& =2 \cdot\left(\sum_{i \in \mathcal{I}_{1}^{(m-1)} \cup \mathcal{I}_{2}^{(m-1)}} \mathscr{C}_{-}^{(m-1)}\left(\mathcal{E}^{(m-1)}, z_{i}^{(m-1)}\right)+2 \cdot \sum_{i \in \mathcal{I}_{3}^{(m-1)} \cup \mathcal{I}_{4}^{(m-1)}} \mathscr{C}_{-}^{(m-1)}\left(\mathcal{E}^{(m-1)}, z_{i}^{(m-1)}\right)\right) \\
& +2 \cdot \sum_{i \in \mathcal{I}_{1}^{(m-1)} \cup \mathcal{I}_{2}^{(m-1)}} \mathscr{C}_{+}^{(m-1)}\left(\mathcal{E}^{(m-1)}, z_{i}^{(m-1)}\right) \\
& =2 \cdot\left(\sum_{i \in \mathcal{I}_{1}^{(m-1)}} \mathscr{C}_{-}^{(m-1)}\left(\mathcal{E}^{(m-1)}, z_{i}^{(m-1)}\right)+\sum_{i \in \mathcal{I}_{2}^{(m-1)}} \mathscr{C}_{-}^{(m-1)}\left(\mathcal{E}^{(m-1)}, z_{i}^{(m-1)}\right)\right) \\
& +4 \cdot\left(\sum_{i \in \mathcal{I}_{3}^{(m-1)}} \mathscr{C}_{-}^{(m-1)}\left(\mathcal{E}^{(m-1)}, z_{i}^{(m-1)}\right)+\sum_{i \in \mathcal{I}_{4}^{(m-1)}} \mathscr{C}_{-}^{(m-1)}\left(\mathcal{E}^{(m-1)}, z_{i}^{(m-1)}\right)\right) \\
& +2 \cdot\left(\sum_{i \in \mathcal{I}_{1}^{(m-1)}} \mathscr{C}_{+}^{(m-1)}\left(\mathcal{E}^{(m-1)}, z_{i}^{(m-1)}\right)+\sum_{i \in \mathcal{I}_{2}^{(m-1)}} \mathscr{C}_{+}^{(m-1)}\left(\mathcal{E}^{(m-1)}, z_{i}^{(m-1)}\right)\right) .
\end{aligned}
$$

Then the lemma follows from Lemma 2.7 and trivial verifications.

Lemma 2.10. For $m \geq 3$,

$$
\nu_{\Upsilon_{(m)}}\left(\mathcal{E}^{(m)}\right)=\frac{194}{3} \cdot 4^{m-1}-\left(4 m^{2}+23 m+\frac{101}{3}-\frac{7 \cdot\left(1+(-1)^{m}\right)}{6}\right) \cdot 2^{m-1} .
$$


Proof. By Lemma 2.1, Lemma 2.3 and Lemma 2.8, we have that for all $k \geq 1$,

$$
\begin{aligned}
\nu_{\Upsilon_{(k)}}\left(\mathcal{E}^{(k)}\right) & =\nu_{\Upsilon_{(k)}}\left(\mathcal{E}_{\ell}^{(k)}\right)+\nu_{\Upsilon_{(k)}}\left(\mathcal{E}_{r}^{(k)}\right)+\nu_{\Upsilon_{(k)}}\left(\mathcal{H}^{(k)}, \mathcal{E}_{\ell}^{(k)}\right)+\nu_{\Upsilon_{(k)}}\left(\mathcal{H}^{(k)}, \mathcal{E}_{r}^{(k)}\right)+\nu_{\Upsilon_{(k)}}\left(\mathcal{H}^{(k)}\right) \\
& =2 \cdot\left(\nu_{\Upsilon_{(k)}}\left(\mathcal{E}_{\ell}^{(k)}\right)+\nu_{(k)}\left(\mathcal{H}^{(k)}, \mathcal{E}_{\ell}^{(k)}\right)\right)+\nu \Upsilon_{(k)}\left(\mathcal{H}^{(k)}\right) \\
& =2 \cdot\left(\nu_{\Upsilon_{(k-1)}}\left(\mathcal{E}^{(k-1)}\right)+\nu_{\Upsilon_{(k)}}\left(\mathcal{H}^{(k)}, \mathcal{E}_{\ell}^{(k)}\right)\right)+\nu_{(k)}\left(\mathcal{H}^{(k)}\right) \\
& =2 \cdot \nu_{\Upsilon_{(k-1)}}\left(\mathcal{E}^{(k-1)}\right)+2 \cdot \nu_{\Upsilon_{(k)}}\left(\mathcal{H}^{(k)}, \mathcal{E}_{\ell}^{(k)}\right)+\left(6 \cdot 4^{k-1}-2^{k+1}\right) .
\end{aligned}
$$

Now we shall prove this lemma by induction on $m$. We first consider the case when $m=3$. Observe

$$
\nu_{\Upsilon_{(1)}}\left(\mathcal{E}^{(1)}\right)=4 \text {. }
$$

By (24), (25) and Lemma 2.9, we have that

$$
\begin{aligned}
\nu_{\Upsilon_{(3)}}\left(\mathcal{E}^{(3)}\right) & =2 \cdot \nu_{\Upsilon_{(2)}}\left(\mathcal{E}^{(2)}\right)+2 \cdot 128+\left(6 \cdot 4^{2}-2^{4}\right) \\
& =2 \cdot\left(2 \cdot \nu_{\Upsilon_{(1)}}\left(\mathcal{E}^{(1)}\right)+2 \cdot 24+6 \cdot 4-2^{3}\right)+336 \\
& =480 \\
& =\frac{194}{3} \cdot 4^{3-1}-\left(4 \cdot 3^{2}+23 \cdot 3+\frac{101}{3}-\frac{7 \cdot\left(1+(-1)^{3}\right)}{6}\right) \cdot 2^{3-1}
\end{aligned}
$$

we are done. Hence, we need to consider the case when $m>3$. By (24) and Lemma 2.9, we have that

$$
\begin{aligned}
\nu_{\Upsilon_{(m)}}\left(\mathcal{E}^{(m)}\right)= & 2 \cdot \nu_{\Upsilon_{(m-1)}}\left(\mathcal{E}^{(m-1)}\right)+2 \cdot \nu_{\Upsilon_{(m)}}\left(\mathcal{H}^{(m)}, \mathcal{E}_{\ell}^{(m)}\right)+\left(6 \cdot 4^{m-1}-2^{m+1}\right) \\
= & 2 \cdot\left(\frac{194}{3} \cdot 4^{m-2}-\left(4(m-1)^{2}+23(m-1)+\frac{101}{3}-\frac{7 \cdot\left(1+(-1)^{m-1}\right)}{6}\right) \cdot 2^{m-2}\right) \\
& +2 \cdot\left(\frac{158}{3} \cdot 4^{m-2}-\left(8 m+\frac{38}{3}+\frac{7 \cdot\left(1+(-1)^{m-1}\right)}{3}\right) \cdot 2^{m-2}\right)+\left(6 \cdot 4^{m-1}-2^{m+1}\right) \\
= & \frac{194}{3} \cdot 4^{m-1}-\left(4 m^{2}+23 m+\frac{101}{3}-\frac{7 \cdot\left(1+(-1)^{m}\right)}{6}\right) \cdot 2^{m-1} .
\end{aligned}
$$

This completes the proof of the lemma.

\section{Proof of Theorem 1.1}

We begin this section by introducing a couple of necessary notations.

Let

$$
\begin{aligned}
& E_{\text {black }}^{n}=\bigcup_{i=1}^{4} E\left(U_{i}^{n} \cup V_{i}^{n}\right), \\
& E_{\text {red }}^{n}=E\left[U_{1}, U_{2}\right] \cup E\left[U_{3}, U_{4}\right] \cup E\left[V_{1}, V_{2}\right] \cup E\left[V_{3}, V_{4}\right], \\
& E_{\text {blue }}^{n}=E\left[U_{1}, U_{3}\right] \cup E\left[U_{2}, U_{4}\right] \cup E\left[V_{1}, V_{3}\right] \cup E\left[V_{2}, V_{4}\right] \cup E\left[U_{1}, V_{3}\right] \cup E\left[U_{2}, V_{4}\right] \cup E\left[V_{1}, U_{3}\right] \cup E\left[V_{2}, U_{4}\right] .
\end{aligned}
$$

By Lemma 2.2, we have the following decomposition

$$
E\left(A Q_{n}\right)=E_{\text {black }}^{n} \cup E_{\text {red }}^{n} \cup E_{\text {blue }}^{n}
$$

with

$$
\begin{aligned}
& E_{\text {black }}^{n}=\left\{e \in E\left(A Q_{n}\right): \operatorname{Dim}(e) \in[-(n-1),-2] \cup[3, n-1]\right\} \\
& E_{\text {red }}^{n}=\left\{e \in E\left(A Q_{n}\right): \operatorname{Dim}(e) \in\{1,2\}\right\} \\
& E_{\text {blue }}^{n}=\left\{e \in E\left(A Q_{n}\right): \operatorname{Dim}(e) \in\{-n, n\}\right\}
\end{aligned}
$$

Let $\mathscr{R} \subseteq \mathbb{R} \times \mathbb{R}$ be a region, and let $F$ be an edge subset of $E\left(A Q_{n}\right)$. We denote by $\operatorname{In}_{\mathscr{R}}(F)$ the part of $F$ which is drawn at the inner of the region $\mathscr{R}$, and by $\operatorname{Out}_{\mathscr{R}}(F)$ the part of $F$ which is drawn outer the region $\mathscr{R}$. If $F=\{e\}$ is a singleton, we simply write $\operatorname{In}_{\mathscr{R}}(e)$ and $\operatorname{Out}_{\mathscr{R}}(e)$ for $\operatorname{In}_{\mathscr{R}}(\{e\})$ and $\operatorname{Out}_{\mathscr{R}}(\{e\})$ respectively.

- For convenience, in the rest of this paper, we shall write $\operatorname{In}(\cdot)$ to mean $\operatorname{In}_{\mathbb{R}_{-5}^{0} \times \mathbb{R}_{5 / 2}^{5}}(\cdot)$ for short.

Now we are in a position to prove Theorem 1.1 For the convenience of readers, we split the proof into two parts, which are put into Subsection 3.1 and Subsection 3.2, respectively. In Subsection 3.1, we give a good drawing $\Gamma_{n}$ of $\operatorname{cr}\left(A Q_{n}\right)$ for all $n \geq 8$. In Subsection 3.2, we shall verify that the crossings of $\Gamma_{n}$ is no more than the upper bound in Theorem [1.1 and therefore completes the proof of Theorem 1.1 . 


\subsection{The drawing $\Gamma_{n}$ for $n \geq 8$}

We first give the drawing of $E_{\text {black }}^{n}$ under $\Gamma_{n}$. In general, $E_{\text {black }}^{n}$ are drawn at the inner of the region $\mathbb{R}_{-5}^{5} \times \mathbb{R}_{-5}^{5}$. Let

$$
\mathcal{W}^{n} \in\left\{U_{1}^{n}, U_{2}^{n}, U_{3}^{n}, U_{4}^{n}, V_{1}^{n}, V_{2}^{n}, V_{3}^{n}, V_{4}^{n}\right\}
$$

Take $\mathcal{N}=5$ and

$$
\left(z_{1}^{\mathcal{N}+(0)}, z_{2}^{\mathcal{N}+(0)}, z_{3}^{\mathcal{N}+(0)}, z_{4}^{\mathcal{N}+(0)}\right)= \begin{cases}(00100,00011,00111,00000), & \text { if } \mathcal{W}^{n}=U_{1}^{n} \\ (00001,00110,00010,00101), & \text { if } \mathcal{W}^{n}=U_{2}^{n} \\ (10100,10011,10111,10000), & \text { if } \mathcal{W}^{n}=U_{3}^{n} \\ (10101,10010,10110,10001), & \text { if } \mathcal{W}^{n}=U_{4}^{n} \\ (01111,01000,01100,01011), & \text { if } \mathcal{W}^{n}=V_{1}^{n} \\ (01010,01101,01001,01110), & \text { if } \mathcal{W}^{n}=V_{2}^{n} \\ (11111,11000,11100,11011), & \text { if } \mathcal{W}^{n}=V_{3}^{n} \\ (11110,11001,11101,11010), & \text { if } \mathcal{W}^{n}=V_{4}^{n}\end{cases}
$$

It is easy to verify that the above values of $z_{1}^{\mathcal{N}+(0)}, z_{2}^{\mathcal{N}+(0)}, z_{3}^{\mathcal{N}+(0)}, z_{4}^{\mathcal{N}+(0)}$ satisfy (7) and (8) with $t_{1}=3=\mathcal{N}-2$ and $t_{2}=-2=-(\mathcal{N}-3)$. Hence, we can draw $\left\langle\mathcal{W}^{n}\right\rangle=\Omega^{(n-\mathcal{N})}\left(\left\{z_{1}^{\mathcal{N}+(0)}, z_{2}^{\mathcal{N}+(0)}, z_{3}^{\mathcal{N}+(0)}, z_{4}^{\mathcal{N}+(0)}\right\}\right)$ as the drawing $\Upsilon_{(n-5)}$ with the initial positive order as $\left(z_{1}^{\mathcal{N}+(0)}, z_{2}^{\mathcal{N}+(0)}, z_{3}^{\mathcal{N}+(0)}, z_{4}^{\mathcal{N}+(0)}\right)$ and satisfying that

$$
X_{z_{1}^{(0)}}=X_{z_{2}^{(0)}}=X_{z_{3}^{(0)}}=X_{z_{4}^{(0)}}= \begin{cases}-2, & \text { if } \mathcal{W}^{n} \in\left\{U_{1}^{n}, U_{3}^{n}\right\} \\ -1, & \text { if } \mathcal{W}^{n} \in\left\{V_{1}^{n}, V_{3}^{n}\right\} \\ 1, & \text { if } \mathcal{W}^{n} \in\left\{V_{2}^{n}, V_{4}^{n}\right\} \\ 2, & \text { if } \mathcal{W}^{n} \in\left\{U_{2}^{n}, U_{4}^{n}\right\}\end{cases}
$$

and

$$
\left(Y_{z_{1}^{(0)}}, Y_{z_{2}^{(0)}}, Y_{z_{3}^{(0)}}, Y_{z_{4}^{(0)}}\right)= \begin{cases}(1,2,3,4), & \text { if } \mathcal{W}^{n} \in\left\{U_{1}^{n}, V_{2}^{n}\right\} \\ (4,3,2,1), & \text { if } \mathcal{W}^{n} \in\left\{V_{1}^{n}, U_{2}^{n}\right\} \\ (-4,-3,-2,-1), & \text { if } \mathcal{W}^{n} \in\left\{U_{3}^{n}, V_{4}^{n}\right\} \\ (-1,-2,-3,-4), & \text { if } \quad \mathcal{W}^{n} \in\left\{V_{3}^{n}, U_{4}^{n}\right\}\end{cases}
$$

We remark that the edges $z_{i}^{(n-5)} z_{j}^{(n-5)} \in \mathcal{E}^{(n-5)}$ will be drawn to be at the left or at the right of the lines $x= \pm 1, \pm 2$ dependent on the locations of $z_{1}^{\mathcal{N}+(0)}, z_{2}^{\mathcal{N}+(0)}, z_{3}^{\mathcal{N}+(0)}, z_{4}^{\mathcal{N}+(0)}$ and $\mathcal{O}\left(z_{i}^{(n-5)} z_{j}^{(n-5)}\right)$ in $\Upsilon_{(n-5)}$.

To proceed with this subsection, we need to rename the vertices of $\mathcal{W}^{n}$ as follows:

$$
\mathcal{W}^{n}= \begin{cases}\left\{u_{1,1}^{n}, u_{1,2}^{n}, \ldots, u_{1,2^{n-3}}^{n}\right\}, & \text { if } \quad \mathcal{W}^{n}=U_{1}^{n} \\ \left\{u_{2,1}^{n}, u_{2,2}^{n}, \ldots, u_{2,2^{n-3}}^{n}\right\}, & \text { if } \quad \mathcal{W}^{n}=U_{2}^{n} \\ \left\{u_{3,1}^{n}, u_{3,2}^{n}, \ldots, u_{3,2^{n-3}}^{n}\right\}, & \text { if } \quad \mathcal{W}^{n}=U_{3}^{n} \\ \left\{u_{4,1}^{n}, u_{4,2}^{n}, \ldots, u_{4,2^{n-3}}^{n}\right\}, & \text { if } \quad \mathcal{W}^{n}=U_{4}^{n} \\ \left\{v_{1,1}^{n}, v_{1,2}^{n}, \ldots, v_{1,2^{n-3}}^{n}\right\}, & \text { if } \quad \mathcal{W}^{n}=V_{1}^{n} \\ \left\{v_{2,1}^{n}, v_{2,2}^{n}, \ldots, v_{2,2^{n-3}}^{n}\right\}, & \text { if } \quad \mathcal{W}^{n}=V_{2}^{n} \\ \left\{v_{3,1}^{n}, v_{3,2}^{n}, \ldots, v_{3,2^{n-3}}^{n}\right\}, & \text { if } \quad \mathcal{W}^{n}=V_{3}^{n} \\ \left\{v_{4,1}^{n}, v_{4,2}^{n}, \ldots, v_{4,2^{n-3}}^{n}\right\}, & \text { if } \quad \mathcal{W}^{n}=V_{4}^{n}\end{cases}
$$

with

$$
\left|Y_{u_{i, 1}^{n}}\right|>\left|Y_{u_{i, 2}^{n}}\right|>\cdots>\left|Y_{u_{i, 2^{n-3}}^{n}}\right|
$$

and

$$
\left|Y_{v_{i, 1}^{n}}\right|>\left|Y_{v_{i, 2}^{n}}\right|>\cdots>\left|Y_{v_{i, 2^{n-3}}^{n}}\right|
$$

where $i \in[1,4]$.

Note that

$$
\left|Y_{u_{1, j}^{n}}\right|=\left|Y_{v_{1, j}^{n}}\right|=\left|Y_{u_{2, j}^{n}}\right|=\left|Y_{v_{2, j}^{n}}\right|=\left|Y_{u_{3, j}^{n}}\right|=\left|Y_{v_{3, j}^{n}}\right|=\left|Y_{u_{4, j}^{n}}\right|=\left|Y_{v_{4, j}^{n}}\right|
$$

for all $j \in\left[1,2^{n-3}\right]$. 
By (9) and (10), we derive that for any $n \geq 8$,

$$
u_{i, j}^{n} \in \Omega^{(n-8)}\left(\left\{u_{i,\left\lceil\frac{j}{2^{n-8}}\right\rceil^{(n)}}^{8}\right\}\right)
$$

and

$$
v_{i, j}^{n} \in \Omega^{(n-8)}\left(\left\{v_{i,\left\lceil\frac{j}{2^{n}-8}\right\rceil}^{8}\right\}\right)
$$

where $i \in[1,4]$ and $j \in\left[1,2^{n-3}\right]$.

By applying Lemma 2.2 repeatedly, we have that

$$
\left\{e \in E\left(A Q_{n}\right): \operatorname{Dim}(e)=-(n-1)\right\}=\left\{u_{i, j}^{n} v_{i, j}^{n}: i \in[1,4], j \in\left[1,2^{n-3}\right]\right\}
$$

and

$$
\left\{e \in E\left(A Q_{n}\right): \operatorname{Dim}(e)=(n-1)\right\}=\left\{u_{i, j}^{n} v_{i, j+(-1)^{j-1}}^{n}: i \in[1,4], j \in\left[1,2^{n-3}\right]\right\} .
$$

By (31), (32), (33), (36) and (37), we can draw the corresponding edges in $\left\{e \in E\left(A Q_{n}\right): \operatorname{Dim}(e) \in\{-(n-\right.$ $1), n-1\}\}$ to be straight lines. Therefore, this completes the characterization of the drawing of $E_{\text {black }}^{n}$.

By applying (12), we see that

$$
\begin{aligned}
\left\{e \in E\left(A Q_{n}\right): \operatorname{Dim}(e)=-(n-2)\right\}= & \left\{u_{i, 2 j-1}^{n} u_{i, 2 j}^{n}: i \in[1,4], j \in\left[1,2^{n-4}\right]\right\} \\
& \cup\left\{v_{i, 2 j-1}^{n} v_{i, 2 j}^{n}: i \in[1,4], j \in\left[1,2^{n-4}\right]\right\} .
\end{aligned}
$$

Now we give the drawing of $E_{r e d}^{n}$ under $\Gamma_{n}$. In general, $E_{\text {red }}^{n}$ are drawn at the inner of the region $\mathbb{R}_{-5}^{5} \times \mathbb{R}_{-5}^{5}$.

By (9) and (10), applying Lemma 2.2 repeatedly, we have that

$$
\begin{aligned}
\left\{e \in E\left(A Q_{n}\right): \operatorname{Dim}(e)=1\right\}= & \left\{u_{1, j}^{n} u_{2, j}^{n}: j \in\left[1,2^{n-3}\right]\right\} \cup\left\{v_{1, j}^{n} v_{2, j}^{n}: j \in\left[1,2^{n-3}\right]\right\} \\
& \cup\left\{u_{3, j}^{n} u_{4, j}^{n}: j \in\left[1,2^{n-3}\right]\right\} \cup\left\{v_{3, j}^{n} v_{4, j}^{n}: j \in\left[1,2^{n-3}\right]\right\}
\end{aligned}
$$

and

$$
\begin{aligned}
\left\{e \in E\left(A Q_{n}\right): \operatorname{Dim}(e)=2\right\}= & \left\{u_{1, j}^{n} u_{2, j+2^{n-4}}^{n}: j \in\left[1,2^{n-3}\right]\right\} \cup\left\{v_{1, j}^{n} v_{2, j+2^{n-4}}^{n}: j \in\left[1,2^{n-3}\right]\right\} \\
& \cup\left\{u_{3, j}^{n} u_{4, j+2^{n-4}}^{n}: j \in\left[1,2^{n-3}\right]\right\} \cup\left\{v_{3, j}^{n} v_{4, j+2^{n-4}}^{n}: j \in\left[1,2^{n-3}\right]\right\},
\end{aligned}
$$

where the subscripts $j+2^{n-4}$ are taken to be the least positive residues modulo $2^{n-3}$.

In general, by (27), (31), (32), (33), (39) and (40), we can draw $E_{\text {red }}^{n}$ satisfying Conditions (i), (ii) and (iii).

(i) The drawing of $E_{r e d}^{n}$ is symmetric with respect to $Y$-axis;

(ii) The drawing of $E_{r e d}^{n}$ is symmetric with respect to $X$-axis;

(iii) $\operatorname{In}_{\mathbb{R}_{-5}^{5} \times \mathbb{R}_{0}^{5}}\left(E_{\text {red }}^{n}\right)$ is symmetric with respect to $y=\frac{5}{2}$.

Take an arbitrary edge $e \in E_{\text {red }}^{n}$. By (39), (40) and Conditions (i)-(iii), we may consider only the case

$$
e \in \bigcup_{t \in\{1,2\}} \mathscr{I}_{t}\left(U_{1,1}^{n} \cup V_{1,1}^{n}\right) .
$$

Then we draw $e$ according to the following inductive rule.

Inductive rule for the drawing of $E_{r e d}^{n}$ : Let $j$ be an arbitrary integer of [1,2 $\left.{ }^{n-4}\right]$. By (34) and (35), we can draw the arcs $\operatorname{In}\left(\mathscr{I}_{1}\left(u_{1, j}^{n}\right)\right), \operatorname{In}\left(\mathscr{I}_{1}\left(v_{1, j}^{n}\right)\right), \operatorname{In}\left(\mathscr{I}_{2}\left(u_{1, j}^{n}\right)\right)$ and $\operatorname{In}\left(\mathscr{I}_{2}\left(v_{1, j}^{n}\right)\right)$ in $\Gamma_{n}$ to be along the original ways of $\operatorname{In}\left(\mathscr{I}_{1}\left(u_{1,\left\lceil\frac{j}{2^{n-8}}\right\rceil}^{8}\right)\right), \operatorname{In}\left(\mathscr{I}_{1}\left(v_{1,\left\lceil\frac{j}{\left.2^{n-8}\right\rceil}\right.}^{8}\right)\right), \operatorname{In}\left(\mathscr{I}_{2}\left(u_{1,\left\lceil\frac{j}{\left.2^{n-8}\right\rceil}\right.}^{8}\right)\right)$ and $\operatorname{In}\left(\mathscr{I}_{2}\left(v_{1,\left\lceil\frac{j}{2^{n-8}}\right\rceil}^{8}\right)\right)$ in $\Gamma_{8}$, respectively.

Therefore, this completes the characterization of the drawing of $E_{r e d}^{n}$. 
Next, we give the drawing of $E_{b l u e}^{n}$ under $\Gamma_{n}$.

By (9) and (10), applying Lemma 2.2 repeatedly, we have that

$$
\begin{aligned}
\left\{e \in E\left(A Q_{n}\right): \operatorname{Dim}(e)=-n\right\}= & \left\{u_{1, j}^{n} v_{3, j}^{n}: j \in\left[1,2^{n-3}\right]\right\} \cup\left\{v_{1, j}^{n} u_{3, j}^{n}: j \in\left[1,2^{n-3}\right]\right\} \\
& \cup\left\{u_{2, j}^{n} v_{4, j}^{n}: j \in\left[1,2^{n-3}\right]\right\} \cup\left\{v_{2, j}^{n} u_{4, j}^{n}: j \in\left[1,2^{n-3}\right]\right\}
\end{aligned}
$$

and

$$
\begin{aligned}
\left\{e \in E\left(A Q_{n}\right): \operatorname{Dim}(e)=n\right\}= & \left\{u_{1, j}^{n} u_{3, j}^{n}: j \in\left[1,2^{n-3}\right]\right\} \cup\left\{v_{1, j}^{n} v_{3, j}^{n}: j \in\left[1,2^{n-3}\right]\right\} \\
& \cup\left\{u_{2, j}^{n} u_{4, j}^{n}: j \in\left[1,2^{n-3}\right]\right\} \cup\left\{v_{2, j}^{n} v_{4, j}^{n}: j \in\left[1,2^{n-3}\right]\right\} .
\end{aligned}
$$
(vi).

In general, by (27), (31), (32), (33), (41) and (42), we can draw $E_{b l u e}^{n}$ satisfying Conditions (iv), (v) and

(iv) The drawing of $E_{b l u e}^{n}$ is symmetric with respect to $Y$-axis;

(v) The drawing of $E_{b l u e}^{n}$ is symmetric with respect to $X$-axis;

(vi) $\operatorname{In}_{\mathbb{R}_{-5}^{5} \times \mathbb{R}_{0}^{5}}\left(E_{\text {blue }}^{n}\right)$ is symmetric with respect to $y=\frac{5}{2}$.

Take an arbitrary edge $e \in E_{\text {blue }}^{n}$. By (41), (42) and Condition (iv), we may assume $e \in \underset{t \in\{-n, n\}}{\bigcup} \mathscr{I}_{t}\left(U_{1}^{n} \cup V_{1}^{n}\right)$. We draw $e$ to be consisting of three nonempty parts, say $\operatorname{In}_{\mathbb{R}_{-5}^{5} \times \mathbb{R}_{0}^{5}}(e), \operatorname{In}_{\mathbb{R}_{-5}^{5} \times \mathbb{R}_{-5}^{0}}(e)$ and $\operatorname{Out}_{\mathbb{R}_{-5}^{5} \times \mathbb{R}_{-5}^{5}}(e)$, of which $\operatorname{In}_{\mathbb{R}_{-5}^{5} \times \mathbb{R}_{0}^{5}}(e)$ and $\operatorname{In}_{\mathbb{R}_{-5}^{5} \times \mathbb{R}_{-5}^{0}}(e)$ 'meet' the line $x=-5$ at right angles, and of which Out $\mathbb{R}_{-5}^{5} \times \mathbb{R}_{-5}^{5}(e)$ connects the two 'points' which are 'produced' by $\operatorname{In}_{\mathbb{R}_{-5}^{5} \times \mathbb{R}_{0}^{5}}(e)$ and $\operatorname{In}_{\mathbb{R}_{-5}^{5} \times \mathbb{R}_{-5}^{0}}(e)$ with the line $x=-5$.

It remains to show the drawing of the other two parts of $e$, that is, $\operatorname{In}_{\mathbb{R}_{-5}^{5} \times \mathbb{R}_{0}^{5}}(e)$ and $\operatorname{In}_{\mathbb{R}_{-5}^{5} \times \mathbb{R}_{-5}^{0}}(e)$. By Conditions (v) and (vi), i.e., the above two kinds of symmetries of the drawing of $E_{b l u e}^{n}$, we can suppose without loss of generality that

$$
e \in \bigcup_{t \in\{-n, n\}} \mathscr{I}_{t}\left(U_{1,1}^{n} \cup V_{1,1}^{n}\right),
$$

and draw $\operatorname{In}(e)$ satisfying the following inductive rule.

Inductive rule for the drawing of $E_{\text {blue }}^{n}$ : Let $j$ be an arbitrary integer of $\left[1,2^{n-4}\right]$. By (34) and (35), we can draw the arc $\operatorname{In}\left(\mathscr{I}_{n}\left(v_{1, j}^{n}\right)\right)$ in $\Gamma_{n}$ to be along the original way of $\operatorname{In}\left(\mathscr{I}_{n}\left(v_{1,\left[\frac{j}{2^{n-8}}\right]}^{8}\right)\right)$ in $\Gamma_{8}$, and we can draw the three $\operatorname{arcs} \operatorname{In}\left(\mathscr{I}_{n}\left(u_{1, j}^{n}\right)\right), \operatorname{In}\left(\mathscr{I}_{-n}\left(u_{1, j}^{n}\right)\right)$ and $\operatorname{In}\left(\mathscr{I}_{-n}\left(v_{1, j}^{n}\right)\right)$ to be a bunch of three arcs in $\Gamma_{n}$ which is along the line $y=Y_{u_{1, j}^{n}}$ and satisfies Property $A$.

Property A: The $\operatorname{arc} \operatorname{In}\left(\mathscr{I}_{-n}\left(u_{1, j}^{n}\right)\right)$ will be drawn precisely at the line $y=Y_{u_{1, j}^{n}}$, one of $\operatorname{In}\left(\mathscr{I}_{n}\left(u_{1, j}^{n}\right)\right)$ and $\operatorname{In}\left(\mathscr{I}_{-n}\left(v_{1, j}^{n}\right)\right)$ lying flat above the line $y=Y_{u_{1, j}^{n}}$ and another below the line $y=Y_{u_{1, j}^{n}}$. In detailed, for $j \in\left[1,2^{n-6}-1\right] \cup\left[2^{n-6}+1,2^{n-6}+2^{n-7}-1\right] \cup\left[2^{n-6}+2^{n-7}+1,2^{n-5}-1\right] \cup\left[2^{n-5}+1,2^{n-5}+2^{n-7}-1\right] \cup\left[2^{n-5}+\right.$ $\left.2^{n-7}+1,2^{n-5}+2^{n-6}-1\right] \cup\left\{2^{n-5}+2^{n-6}+1\right\}, \operatorname{In}\left(\mathscr{I}_{-n}\left(v_{1, j}^{n}\right)\right)$ is drawn above the line $y=Y_{u_{1, j}^{n}}$, for otherwise, $\operatorname{In}\left(\mathscr{I}_{-n}\left(v_{1, j}^{n}\right)\right)$ is drawn below the line $y=Y_{u_{1, j}^{n}}$.

Therefore, this completes the characterization of the drawing of $E_{b l u e}^{n}$.

Combined (28), (29), (30) and Conditions (i)-(vi), we conclude that $\Gamma_{n}$ has Property B.

\section{Property B:}

- $\Gamma_{n}$ is symmetric with respect to $Y$-axis;

- $\Gamma_{n}$ is symmetric with respect to $X$-axis;

- $\operatorname{In}_{\mathbb{R}_{-5}^{5} \times \mathbb{R}_{0}^{5}}\left(E\left(A Q_{n}\right)\right)$ is symmetric with respect to $y=\frac{5}{2}$.

This completes the characterizations of the drawing $\Gamma_{n}$. To make the above process clear, we give the drawings of $\operatorname{In}\left(A Q_{n}\right)$ under $\Gamma_{n}$ for $n=8,9$ in Figure 3.1 and 3.2, respectively. 


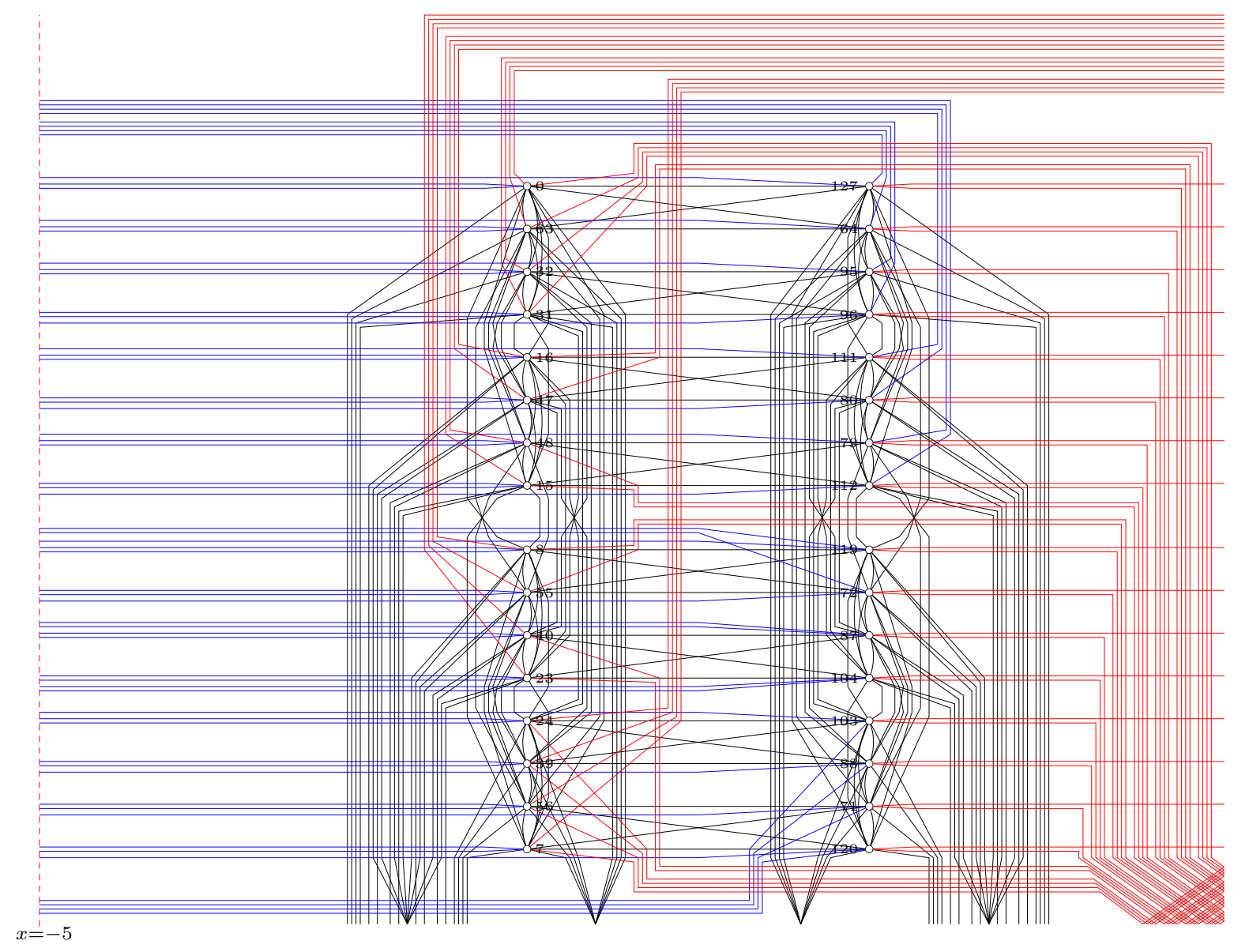

Figure 3.1: $\operatorname{In}\left(A Q_{8}\right)$ in the drawing $\Gamma_{8}$

- For the clearness of composition, in the rest of this paper, any vertex $a=a_{n} a_{n-1} \cdots a_{1} \in V\left(A Q_{n}\right)$ in figures will be represented by the corresponding decimal number $a_{n} \cdot 2^{n-1}+a_{n-1} \cdot 2^{n-2}+\cdots+a_{1}$. 


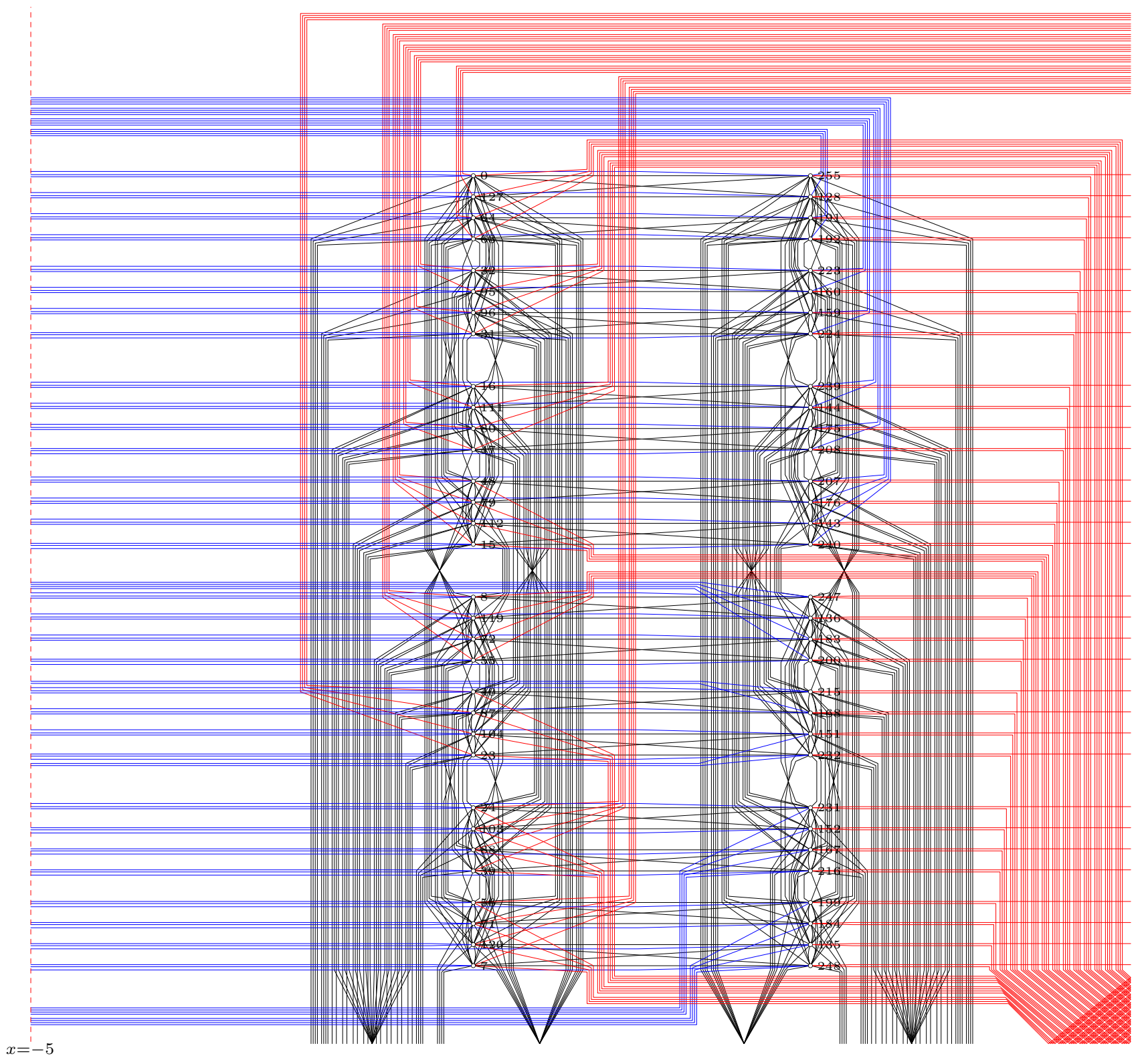

Figure 3.2: $\operatorname{In}\left(A Q_{9}\right)$ in the drawing $\Gamma_{9}$ 


\subsection{The calculation of crossings in $\Gamma_{n}$}

In this subsection, we shall calculate the number of crossings of $A Q_{n}$ in the drawing $\Gamma_{n}$ for $n \geq 8$. By (26) and Lemma 2.1. we have that

$$
\begin{aligned}
\nu_{\Gamma_{n}}\left(A Q_{n}\right)= & \nu_{\Gamma_{n}}\left(E_{\text {blue }}^{n}\right)+\nu_{\Gamma_{n}}\left(E_{\text {red }}^{n}\right)+\nu_{\Gamma_{n}}\left(E_{\text {black }}^{n}\right) \\
& +\nu_{\Gamma_{n}}\left(E_{\text {blue }}^{n}, E_{\text {red }}^{n}\right)+\nu_{\Gamma_{n}}\left(E_{\text {blue }}^{n}, E_{\text {black }}^{n}\right)+\nu_{\Gamma_{n}}\left(E_{\text {red }}^{n}, E_{\text {black }}^{n}\right) .
\end{aligned}
$$

By (43), the process of calculation can be divided into six parts. To begin with the six parts, we need some preliminaries.

For $i \in[1,4]$, let

$$
\begin{aligned}
U_{i, 1}^{n} & =\left\{u_{i, 1}^{n}, u_{i, 2}^{n}, \ldots, u_{i, 2^{n-4}}^{n}\right\}, \\
U_{i, 2}^{n} & =\left\{u_{i, 2^{n-4}+1}^{n}, u_{i, 2^{n-4}+2}^{n}, \ldots, u_{i, 2^{n-3}}^{n}\right\} \\
V_{i, 1}^{n} & =\left\{v_{i, 1}^{n}, v_{i, 2}^{n}, \ldots, v_{i, 2^{n-4}}^{n}\right\} \\
V_{i, 2}^{n} & =\left\{v_{i, 2^{n-4}+1}^{n}, v_{i, 2^{n-4}+2}^{n}, \ldots, v_{i, 2^{n-3}}^{n}\right\} .
\end{aligned}
$$

For any edge $e \in \bigcup_{t \in\{-n, n\}} \mathscr{I}_{t}\left(V_{1,1}^{n}\right)$, let $Y^{e}$ be the $Y$-coordinate of the 'point' at which $\operatorname{In}(e)$ and the line $x=-2$ cross each other, and let $\mathcal{L}(\operatorname{In}(e))$ be the least positive integer $j \in\left[1,2^{n-4}+1\right]$ such that $Y^{e}>Y_{u_{1, j}^{n}}$.

Let

$$
H_{\text {red }}^{n}=\bigcup_{t \in[1,2]} \mathscr{I}_{t}\left(U_{1,1}^{n}\right)
$$

and

$$
H_{\text {black }}^{n}=\bigcup_{t \in[-(n-1),-2] \cup[3, n-1]} \mathscr{I}_{t}\left(U_{1,1}^{n}\right) .
$$

Take an edge $e \in H_{\text {red }}^{n} \cup H_{\text {black }}^{n}$. We say $e$ covers vertex $u_{1, j}^{n}$ for some $j \in\left[1,2^{n-4}\right]$ provided that $\operatorname{In}(e)$ crosses the line $y=Y_{u_{1, j}^{n}}$. Suppose $e$ is incident to $u_{1, t}^{n}$ for some $t \in\left[1,2^{n-4}\right]$. Let $\varepsilon\left(e, u_{1, t}^{n}\right)=1\left(\varepsilon\left(e, u_{1, t}^{n}\right)=-1\right)$ if $e$ shots upward (downward) of the line $y=Y_{u_{1, t}^{n}}$. For example, $\varepsilon\left(\mathscr{I}_{2}\left(u_{1, j}^{8}\right), u_{1, j}^{8}\right)=1$ for $j \in[1,6]$ and $\varepsilon\left(\mathscr{I}_{2}\left(u_{1, k}^{8}\right), u_{1, k}^{8}\right)=-1$ for $k \in[7,8]$ (see Figure 3.1).

Take an edge subset $H \subseteq H_{\text {red }}^{n} \cup H_{\text {black }}^{n}$. Then we define

$$
\begin{gathered}
\varpi_{j}^{n, H}=\mid\left\{e \in H: e \text { covers } u_{1, j}^{n}\right\} \mid \\
\vartheta_{j}^{n, H}=\mid\left\{e \in H: e \text { is incident to } u_{1, j}^{n} \text { with } \varepsilon\left(e, u_{1, j}^{n}\right)=1\right\} \mid
\end{gathered}
$$

and

$$
\varsigma_{j}^{n, H}=\mid\left\{e \in H: e \text { is incident to } u_{1, j}^{n} \text { with } \varepsilon\left(e, u_{1, j}^{n}\right)=-1\right\} \mid .
$$

Therefore, it is easy to derive the following

Assertion C. Let $e^{\prime} \in \bigcup_{t \in\{-n, n\}} \mathscr{I}_{t}\left(V_{1,1}^{n}\right)$, and let $H$ be an edge subset of $H_{\text {red }}^{n} \cup H_{\text {black }}^{n}$. If $\operatorname{In}\left(e^{\prime}\right)$ is drawn along the line $y=Y_{u_{1, \mathcal{L}(\operatorname{In}(e))}}$ then

$$
\left|\left\{e \in H: \nu_{\Gamma_{n}}\left(\operatorname{In}(e), \operatorname{In}\left(e^{\prime}\right)\right)=1\right\}\right|=\varpi_{\mathcal{L}\left(\operatorname{In}\left(e^{\prime}\right)\right)}^{n, H}+\vartheta_{\mathcal{L}\left(\operatorname{In}\left(e^{\prime}\right)\right)}^{n, H},
$$

if $\operatorname{In}\left(e^{\prime}\right)$ is drawn along the line $y=Y_{u_{1, \mathcal{L}\left(\operatorname{In}\left(e^{\prime}\right)\right)-1}^{n}}$ then

$$
\left|\left\{e \in H: \nu_{\Gamma_{n}}\left(\operatorname{In}(e), \operatorname{In}\left(e^{\prime}\right)\right)=1\right\}\right|=\varpi_{\mathcal{L}\left(\operatorname{In}\left(e^{\prime}\right)\right)-1}^{n, H}+\varsigma_{\mathcal{L}\left(\operatorname{In}\left(e^{\prime}\right)\right)-1}^{n, H}
$$

The following definitions will be key for the computations of $\nu_{\Gamma_{n}}\left(E_{\text {blue }}^{n}, E_{\text {red }}^{n}\right)$ and $\nu_{\Gamma_{n}}\left(E_{\text {blue }}^{n}, E_{\text {black }}^{n}\right)$ later.

Define

$$
s_{n, j}=\varpi_{j}^{n, H_{r e d}^{n}}+\vartheta_{j}^{n, H_{r e d}^{n}}
$$




$$
t_{n, j}=\varpi_{j}^{n, H_{\text {black }}^{n}}+\vartheta_{j}^{n, H_{\text {black }}^{n}}
$$

and

$$
t_{n, j}^{\prime}=\varpi_{j}^{n, E\left(U_{1}^{n}\right)}+\vartheta_{j}^{n, E\left(U_{1}^{n}\right)}
$$

where $j \in\left[1,2^{n-4}\right]$, and define

$$
\begin{gathered}
s_{n, 2^{n-4}+1}=\varpi_{2^{n-4}}^{n, H_{r e d}^{n}}+\varsigma_{2^{n-4}}^{n, H_{r e d}^{n}}, \\
t_{n, 2^{n-4}+1}=\varpi_{2^{n-4}}^{n, H_{b l a c k}^{n}}+\varsigma_{2^{n-4}}^{n, H_{b l a c k}^{n}}
\end{gathered}
$$

and

$$
t_{n, 2^{n-4}+1}^{\prime}=\varpi_{2^{n-4}}^{n, E\left(U_{1}^{n}\right)}+\varsigma_{2^{n-4}}^{n, E\left(U_{1}^{n}\right)} .
$$

Since $H_{\text {black }}^{n} \backslash E\left(U_{1}^{n}\right)=\bigcup_{t \in\{-(n-1), n-1\}} \mathscr{I}_{t}\left(U_{1,1}^{n}\right)$, it follows from (36) and (37) that

$$
t_{n, j}=\left\{\begin{array}{lll}
t_{n, j}^{\prime}, & \text { if } j \equiv 1 \quad(\bmod 2) \\
t_{n, j}^{\prime}+1, & \text { if } j \equiv 0 & (\bmod 2)
\end{array}\right.
$$

and

$$
\varsigma_{j}^{n, H_{b l a c k}^{n}}=\left\{\begin{array}{lll}
\varsigma_{j}^{n, E\left(U_{1}^{n}\right)}+1, & \text { if } j \equiv 1 & (\bmod 2) \\
\varsigma_{j}^{n, E\left(U_{1}^{n}\right)}, & \text { if } j \equiv 0 & (\bmod 2)
\end{array}\right.
$$

for all $j \in\left[1,2^{n-4}+1\right]$.

Lemma 3.1. Let $n \geq 8$ be an integer. Then the following conclusions hold.

$$
\begin{aligned}
& \text { 1. } s_{n, j+1}=\varpi_{j}^{n, H_{r e d}^{n}}+\varsigma_{j}^{n, H_{r e d}^{n}} \quad \text { for all } j \in\left[1,2^{n-4}\right] \text {; } \\
& \text { 2. } s_{n, 2 j-1}=2 s_{n-1, j} \quad \text { for all } j \in\left[1,2^{n-5}+1\right] \text {; } \\
& \text { 3. } s_{n, 2 j}=s_{n-1, j}+s_{n-1, j+1} \quad \text { for all } j \in\left[1,2^{n-5}\right] \text {; } \\
& \text { 4. } t_{n, j+1}=\varpi_{j}^{n, H_{b l a c k}^{n}}+\varsigma_{j}^{n, H_{\text {black }}^{n}} \quad \text { for all } j \in\left[1,2^{n-4}-1\right] \text {; } \\
& \text { 5. } t_{n, 2 j-1}=2 t_{n-1, j} \quad \text { for all } j \in\left[1,2^{n-5}+1\right] \text {; } \\
& \text { 6. } t_{n, 2 j}=t_{n-1, j}+t_{n-1, j+1}+2 \text { for all } j \in\left[1,2^{n-5}\right] \text {. }
\end{aligned}
$$

Proof. To prove Conclusion 1, 2 and 3, we shall need the following notations. Let

$$
s_{n, j}^{d}=\varpi_{j}^{n, H_{r e d}^{n}}+\varsigma_{j}^{n, H_{r e d}^{n}} \quad \text { for } j \in\left[1,2^{n-4}\right] .
$$

We have that for $n>8$ and for $j \in\left[1,2^{n-5}\right]$,

$$
\begin{aligned}
s_{n, 2 j-1}^{d}= & \varpi_{2 j-1}^{n, H_{r e d}^{n}}+\varsigma_{2 j-1}^{n, H_{r e d}^{n}} \\
= & \left(2 \cdot \varpi_{j}^{n-1, H_{r e d}^{n-1}}+\vartheta_{2 j}^{n, H_{r e d}^{n}}\right)+\varsigma_{2 j-1}^{n, H_{r e d}^{n}} \\
= & \left(2 \cdot \varpi_{j}^{n-1, H_{r e d}^{n-1}}+\vartheta_{j}^{n-1, H_{r e d}^{n-1}}\right)+\varsigma_{j}^{n-1, H_{r e d}^{n-1}} \\
= & \left(\varpi_{j}^{n-1, H_{r e d}^{n-1}}+\vartheta_{j}^{n-1, H_{r e d}^{n-1}}\right)+\left(\varpi_{j}^{n-1, H_{r e d}^{n-1}}+\varsigma_{j}^{n-1, H_{r e d}^{n-1}}\right) \\
= & s_{n-1, j}+s_{n-1, j}^{d}, \\
s_{n, 2 j}^{d} & =\varpi_{2 j}^{n, H_{r e d}^{n}}+\varsigma_{2 j}^{n, H_{r e d}^{n}} \\
& =\left(2 \cdot \varpi_{j}^{n-1, H_{\text {red }}^{n-1}}+\varsigma_{2 j-1}^{n, H_{r e d}^{n}}\right)+\varsigma_{j}^{n-1, H_{r e d}^{n-1}} \\
& =\left(2 \cdot \varpi_{j}^{n-1, H_{\text {red }}^{n-1}}+\varsigma_{j}^{n-1, H_{r e d}^{n-1}}\right)+\varsigma_{j}^{n-1, H_{r e d}^{n-1}} \\
& =2 \cdot\left(\varpi_{j}^{n-1, H_{\text {red }}^{n-1}}+\varsigma_{j}^{n-1, H_{r e d}^{n-1}}\right) \\
& =2 \cdot s_{n-1, j}^{d},
\end{aligned}
$$




$$
\begin{aligned}
s_{n, 2 j-1} & =\varpi_{2 j-1}^{n, H_{\text {red }}^{n}}+\vartheta_{2 j-1}^{n, H_{\text {red }}^{n}} \\
& =\left(2 \cdot \varpi_{j}^{n-1, H_{\text {red }}^{n-1}}+\vartheta_{2 j}^{n, H_{\text {red }}^{n}}\right)+\vartheta_{j}^{n-1, H_{\text {red }}^{n-1}} \\
& =\left(2 \cdot \varpi_{j}^{n-1, H_{\text {red }}^{n-1}}+\vartheta_{j}^{n-1, H_{\text {red }}^{n-1}}\right)+\vartheta_{j}^{n-1, H_{\text {red }}^{n-1}} \\
& =2 \cdot\left(\varpi_{j}^{n-1, H_{\text {red }}^{n-1}}+\vartheta_{j}^{n-1, H_{\text {red }}^{n-1}}\right) \\
& =2 \cdot s_{n-1, j}
\end{aligned}
$$

and

$$
\begin{aligned}
s_{n, 2 j} & =\varpi_{2 j}^{n, H_{\text {red }}^{n}}+\vartheta_{2 j}^{n, H_{\text {red }}^{n}} \\
& =\left(2 \cdot \varpi_{j}^{n-1, H_{\text {red }}^{n-1}}+\varsigma_{2 j-1}^{n, H_{\text {red }}^{n}}\right)+\vartheta_{j}^{n-1, H_{\text {red }}^{n-1}} \\
& =\left(2 \cdot \varpi_{j}^{n-1, H_{\text {red }}^{n-1}}+\varsigma_{j}^{n-1, H_{\text {red }}^{n-1}}\right)+\vartheta_{j}^{n-1, H_{\text {red }}^{n-1}} \\
& =\left(\varpi_{j}^{n-1, H_{\text {red }}^{n-1}}+\vartheta_{j}^{n-1, H_{\text {red }}^{n-1}}\right)+\left(\varpi_{j}^{n-1, H_{\text {red }}^{n-1}}+\varsigma_{j}^{n-1, H_{\text {red }}^{n-1}}\right) \\
& =s_{n-1, j}+s_{n-1, j}^{d} .
\end{aligned}
$$

Now we prove Conclusion 1 by induction on $n$. If $n=8$, it follows from trivial verifications. Next we need only to consider the case when $n>8$. Conclusion 1 is equivalent to show that

$$
s_{n, j+1}=s_{n, j}^{d} \quad \text { for all } j \in\left[1,2^{n-4}-1\right] .
$$

By (54), (55), (56), (57) and the induction hypothesis, we have that for all $j \in\left[1,2^{n-5}-1\right]$,

$$
\begin{aligned}
s_{n, 2 j+1} & =2 \cdot s_{n-1, j+1} \\
& =2 \cdot s_{n-1, j}^{d} \\
& =s_{n, 2 j}^{d},
\end{aligned}
$$

and that for all $j \in\left[1,2^{n-5}\right]$,

$$
\begin{aligned}
s_{n, 2 j} & =s_{n-1, j}+s_{n-1, j}^{d} \\
& =s_{n, 2 j-1}^{d},
\end{aligned}
$$

and Conclusion 1 follows immediately.

Furthermore, Conclusions 2 and 3 follow from (56), (57) and Conclusion 1.

Now we prove Conclusions 4, 5 and 6. By (29), we can infer that

$$
\varpi_{j+1}^{n, E\left(U_{1}^{n}\right)}+\vartheta_{j+1}^{n, E\left(U_{1}^{n}\right)}=\varpi_{j}^{n, E\left(U_{1}^{n}\right)}+\varsigma_{j}^{n, E\left(U_{1}^{n}\right)} \quad \text { for all } j \in\left[1,2^{n-4}\right] .
$$

It follows from (52), (53) and (58) that for all $j \in\left[1,2^{n-4}-1\right]$,

if $j \equiv 1(\bmod 2)$ then

$$
\begin{aligned}
& t_{n, j+1}=t_{n, j+1}^{\prime}+1 \\
& =\varpi_{j+1}^{n, E\left(U_{1}^{n}\right)}+\vartheta_{j+1}^{n, E\left(U_{1}^{n}\right)}+1 \\
& =\varpi_{j}^{n, E\left(U_{1}^{n}\right)}+\varsigma_{j}^{n, E\left(U_{1}^{n}\right)}+1 \\
& =\varpi_{j}^{n, H_{\text {black }}^{n}}+\varsigma_{j}^{n, E\left(U_{1}^{n}\right)}+1 \\
& =\varpi_{j}^{n, H_{\text {black }}^{n}}+\varsigma_{j}^{n, H_{\text {black }}^{n}}
\end{aligned}
$$


if $j \equiv 0(\bmod 2)$ then

$$
\begin{aligned}
t_{n, j+1} & =t_{n, j+1}^{\prime} \\
& =\varpi_{j+1}^{n, E\left(U_{1}^{n}\right)}+\vartheta_{j+1}^{n, E\left(U_{1}^{n}\right)} \\
& =\varpi_{j}^{n, E\left(U_{1}^{n}\right)}+\varsigma_{j}^{n, E\left(U_{1}^{n}\right)} \\
& =\varpi_{j}^{n, H_{\text {black }}^{n}}+\varsigma_{j}^{n, E\left(U_{1}^{n}\right)} \\
& =\varpi_{j}^{n, H_{\text {black }}^{n}}+\varsigma_{j}^{n, H_{\text {black }}^{n},}
\end{aligned}
$$

and thus, Conclusion 4 follows.

It remains to show Conclusions 5 and 6. By (36), (37), (38), (52), (58), Conclusion 4 and Lemma 2.2, we conclude that for all $j \in\left[1,2^{n-5}\right]$,

if $j \equiv 1(\bmod 2)$ then

$$
\begin{aligned}
t_{n, 2 j-1} & =t_{n, 2 j-1}^{\prime} \\
& =\varpi_{2 j-1}^{n, E\left(U_{1}^{n}\right)}+\vartheta_{2 j-1}^{n, E\left(U_{1}^{n}\right)} \\
& =\left(2 \cdot \varpi_{j}^{n-1, E\left(U_{1}^{n-1}\right)}+\vartheta_{2 j}^{n, E\left(U_{1}^{n}\right)}-1\right)+\vartheta_{j}^{n-1, E\left(U_{1}^{n-1}\right)} \\
& =\left(2 \cdot \varpi_{j}^{n-1, E\left(U_{1}^{n-1}\right)}+\left(\vartheta_{j}^{n-1, E\left(U_{1}^{n-1}\right)}+1\right)-1\right)+\vartheta_{j}^{n-1, E\left(U_{1}^{n-1}\right)} \\
& =2 \cdot\left(\varpi_{j}^{n-1, E\left(U_{1}^{n-1}\right)}+\vartheta_{j}^{n-1, E\left(U_{1}^{n-1}\right)}\right) \\
& =2 \cdot t_{n, j}^{\prime} \\
& =2 \cdot t_{n, j}
\end{aligned}
$$

and

$$
\begin{aligned}
t_{n, 2 j} & =t_{n, 2 j}^{\prime}+1 \\
& =\varpi_{2 j}^{n, E\left(U_{1}^{n}\right)}+\vartheta_{2 j}^{n, E\left(U_{1}^{n}\right)}+1 \\
& =\left(2 \cdot \varpi_{j}^{n-1, E\left(U_{1}^{n-1}\right)}+\varsigma_{2 j-1}^{n, E\left(U_{1}^{n}\right)}-1\right)+\left(\vartheta_{j}^{n-1, E\left(U_{1}^{n-1}\right)}+1\right)+1 \\
& =\left(2 \cdot \varpi_{j}^{n-1, E\left(U_{1}^{n-1}\right)}+\left(\varsigma_{j}^{n-1, E\left(U_{1}^{n-1}\right)}+2\right)-1\right)+\left(\vartheta_{j}^{n-1, E\left(U_{1}^{n-1}\right)}+1\right)+1 \\
& =\left(\varpi_{j}^{n-1, E\left(U_{1}^{n-1}\right)}+\vartheta_{j}^{n-1, E\left(U_{1}^{n-1}\right)}\right)+\left(\varpi_{j}^{n-1, E\left(U_{1}^{n-1}\right)}+\varsigma_{j}^{n-1, E\left(U_{1}^{n-1}\right)}\right)+3 \\
& =\left(\varpi_{j}^{n-1, E\left(U_{1}^{n-1}\right)}+\vartheta_{j}^{n-1, E\left(U_{1}^{n-1}\right)}\right)+\left(\varpi_{j+1}^{n-1, E\left(U_{1}^{n-1}\right)}+\vartheta_{j+1}^{n-1, E\left(U_{1}^{n-1}\right)}\right)+3 \\
& =t_{n, j}^{\prime}+\left(t_{n, j+1}^{\prime}+1\right)+2 \\
& =t_{n, j}+t_{n, j+1}+2,
\end{aligned}
$$

if $j \equiv 0(\bmod 2)$ then

$$
\begin{aligned}
t_{n, 2 j-1} & =t_{n, 2 j-1}^{\prime} \\
& =\varpi_{2 j-1}^{n, E\left(U_{1}^{n}\right)}+\vartheta_{2 j-1}^{n, E\left(U_{1}^{n}\right)} \\
& =\left(2 \cdot \varpi_{j}^{n-1, E\left(U_{1}^{n-1}\right)}+\vartheta_{2 j}^{n, E\left(U_{1}^{n}\right)}-1\right)+\left(\vartheta_{j}^{n-1, E\left(U_{1}^{n-1}\right)}+1\right) \\
& =\left(2 \cdot \varpi_{j}^{n-1, E\left(U_{1}^{n-1}\right)}+\left(\vartheta_{j}^{n-1, E\left(U_{1}^{n-1}\right)}+2\right)-1\right)+\left(\vartheta_{j}^{n-1, E\left(U_{1}^{n-1}\right)}+1\right) \\
& =2 \cdot\left(\varpi_{j}^{n-1, E\left(U_{1}^{n-1}\right)}+\vartheta_{j}^{n-1, E\left(U_{1}^{n-1}\right)}\right)+2 \\
& =2 \cdot t_{n, j}^{\prime}+2 \\
& =2 \cdot\left(t_{n, j}^{\prime}+1\right) \\
& =2 \cdot t_{n, j}
\end{aligned}
$$


and

$$
\begin{aligned}
t_{n, 2 j} & =t_{n, 2 j}^{\prime}+1 \\
& =\varpi_{2 j}^{n, E\left(U_{1}^{n}\right)}+\vartheta_{2 j}^{n, E\left(U_{1}^{n}\right)}+1 \\
& =\left(2 \cdot \varpi_{j}^{n-1, E\left(U_{1}^{n-1}\right)}+\varsigma_{2 j-1}^{n, E\left(U_{1}^{n}\right)}-1\right)+\left(\vartheta_{j}^{n-1, E\left(U_{1}^{n-1}\right)}+2\right)+1 \\
& =\left(2 \cdot \varpi_{j}^{n-1, E\left(U_{1}^{n-1}\right)}+\left(\varsigma_{j}^{n-1, E\left(U_{1}^{n-1}\right)}+1\right)-1\right)+\left(\vartheta_{j}^{n-1, E\left(U_{1}^{n-1}\right)}+2\right)+1 \\
& =\left(\varpi_{j}^{n-1, E\left(U_{1}^{n-1}\right)}+\vartheta_{j}^{n-1, E\left(U_{1}^{n-1}\right)}\right)+\left(\varpi_{j}^{n-1, E\left(U_{1}^{n-1}\right)}+\varsigma_{j}^{n-1, E\left(U_{1}^{n-1}\right)}\right)+3 \\
& =\left(\varpi_{j}^{n-1, E\left(U_{1}^{n-1}\right)}+\vartheta_{j}^{n-1, E\left(U_{1}^{n-1}\right)}\right)+\left(\varpi_{j+1}^{n-1, E\left(U_{1}^{n-1}\right)}+\vartheta_{j+1}^{n-1, E\left(U_{1}^{n-1}\right)}\right)+3 \\
& =t_{n, j}^{\prime}+t_{n, j+1}^{\prime}+3 \\
& =t_{n, j}^{\prime}+\left(t_{n, j+1}^{\prime}+1\right)+2 \\
& =t_{n, j}+t_{n, j+1}+2,
\end{aligned}
$$

and thus, Conclusions 5 and 6 follow immediately.

1. Calculation of $\nu_{\Gamma_{n}}\left(E_{\text {blue }}^{n}\right)$.

By (27), (41), (42) and Property B, we have that

$$
\begin{aligned}
& \nu_{\Gamma_{n}}\left(E_{\text {blue }}^{n}\right) \\
= & \nu_{\Gamma_{n}}\left(\operatorname{Out}_{\mathbb{R}_{-5}^{5} \times \mathbb{R}_{-5}^{5}}\left(E_{\text {blue }}^{n}\right)\right)+\nu_{\Gamma_{n}}\left(\operatorname{In}_{\mathbb{R}_{-5}^{5} \times \mathbb{R}_{-5}^{5}}\left(E_{\text {blue }}^{n}\right)\right) \\
= & \nu_{\Gamma_{n}}\left(\operatorname{Out}_{\mathbb{R}_{-5}^{5} \times \mathbb{R}_{-5}^{5}}\left(E_{\text {blue }}^{n}\right)\right)+8 \cdot \nu_{\Gamma_{n}}\left(\operatorname{In}\left(E_{\text {blue }}^{n}\right)\right) \\
= & 2 \cdot \nu_{\Gamma_{n}}\left(\operatorname{Out}_{\mathbb{R}_{-5}^{5} \times \mathbb{R}_{-5}^{5}}\left(\bigcup_{t \in\{-n, n\}} \mathscr{I}_{t}\left(U_{1}^{n} \cup V_{1}^{n}\right)\right)\right)+8 \cdot \nu_{\Gamma_{n}}\left(\operatorname{In}\left(\bigcup_{t \in\{-n, n\}} \mathscr{I}_{t}\left(U_{1,1}^{n} \cup V_{1,1}^{n}\right)\right)\right) .
\end{aligned}
$$

Now we calculate $\nu_{\Gamma_{n}}\left(\mathrm{Out}_{\mathbb{R}_{-5}^{5} \times \mathbb{R}_{-5}^{5}}\left(\bigcup_{t \in\{-n, n\}} \mathscr{I}_{t}\left(U_{1}^{n} \cup V_{1}^{n}\right)\right)\right)$.

By (41), (42), Inductive rule for the drawing of $E_{b l u e}^{n}$ and Property B, we have that

$$
\begin{gathered}
\nu_{\Gamma_{n}}\left(\operatorname{Out}_{\mathbb{R}_{-5}^{5} \times \mathbb{R}_{-5}^{5}}\left(\mathscr{I}_{n}\left(V_{1}^{n}\right)\right)\right)=0, \\
\nu_{\Gamma_{n}}\left(\operatorname{Out}_{\mathbb{R}_{-5}^{5} \times \mathbb{R}_{-5}^{5}}\left(\mathscr{I}_{n}\left(V_{1}^{n}\right)\right), \operatorname{Out}_{\mathbb{R}_{-5}^{5} \times \mathbb{R}_{-5}^{5}}\left(\mathscr{I}_{-n}\left(V_{1}^{n}\right) \cup \mathscr{I}_{-n}\left(U_{1}^{n}\right) \cup \mathscr{I}_{n}\left(U_{1}^{n}\right)\right)\right)=0,
\end{gathered}
$$

and that for any two distinct integers $j, k \in\left[1,2^{n-3}\right]$,

$$
\nu_{\Gamma_{n}}\left(\operatorname{Out}_{\mathbb{R}_{-5}^{5} \times \mathbb{R}_{-5}^{5}}\left(\mathscr{I}_{-n}\left(v_{1, j}^{n}\right) \cup \mathscr{I}_{-n}\left(u_{1, j}^{n}\right) \cup \mathscr{I}_{n}\left(u_{1, j}^{n}\right)\right), \operatorname{Out}_{\mathbb{R}_{-5}^{5} \times \mathbb{R}_{-5}^{5}}\left(\mathscr{I}_{-n}\left(v_{1, k}^{n}\right) \cup \mathscr{I}_{-n}\left(u_{1, k}^{n}\right) \cup \mathscr{I}_{n}\left(u_{1, k}^{n}\right)\right)\right)=0
$$

and furthermore that for every $j \in\left[1,2^{n-3}\right]$,

$$
\nu_{\Gamma_{n}}\left(\operatorname{Out}_{\mathbb{R}_{-5}^{5} \times \mathbb{R}_{-5}^{5}}\left(\mathscr{I}_{-n}\left(v_{1, j}^{n}\right) \cup \mathscr{I}_{-n}\left(u_{1, j}^{n}\right) \cup \mathscr{I}_{n}\left(u_{1, j}^{n}\right)\right)\right)=1
$$

which are shown in Figure 3.3 for example. In Figure 3.3, $P_{u_{1, j}^{n}}^{\epsilon}\left(P_{v_{1, j}^{n}}^{\epsilon}\right)$ denotes the 'point' at which $\operatorname{In}_{\mathbb{R}_{-5}^{5} \times \mathbb{R}_{0}^{5}}\left(\mathscr{I}_{\epsilon}\left(u_{1, j}^{n}\right)\right)$ $\left(\operatorname{In}_{\mathbb{R}_{-5}^{5} \times \mathbb{R}_{0}^{5}}\left(\mathscr{I}_{\epsilon}\left(v_{1, j}^{n}\right)\right)\right)$ and the line $x=-5$ cross each other, and $P_{u_{3, j}^{n}}^{\epsilon}\left(P_{v_{3, j}^{n}}^{\epsilon}\right)$ denotes the 'point' at which $\operatorname{In}_{\mathbb{R}_{-5}^{5} \times \mathbb{R}_{-5}^{0}}\left(\mathscr{I}_{\epsilon}\left(u_{1, j}^{n}\right)\right) \quad\left(\operatorname{In}_{\mathbb{R}_{-5}^{5} \times \mathbb{R}_{-5}^{0}}\left(\mathscr{I}_{\epsilon}\left(v_{1, j}^{n}\right)\right)\right)$ and the line $x=-5$ cross each other, where $\epsilon \in\{-n, n\}$.

Combined (65), (66), (67) and (68), we conclude that

$$
\nu_{\Gamma_{n}}\left(\operatorname{Out}_{\mathbb{R}_{-5}^{5} \times \mathbb{R}_{-5}^{5}}\left(\bigcup_{t \in\{-n, n\}} \mathscr{I}_{t}\left(U_{1}^{n} \cup V_{1}^{n}\right)\right)\right)=2^{n-3} .
$$

Next we calculate $\nu_{\Gamma_{n}}\left(\operatorname{In}\left(\bigcup_{t \in\{-n, n\}} \mathscr{I}_{t}\left(U_{1,1}^{n} \cup V_{1,1}^{n}\right)\right)\right)$. 


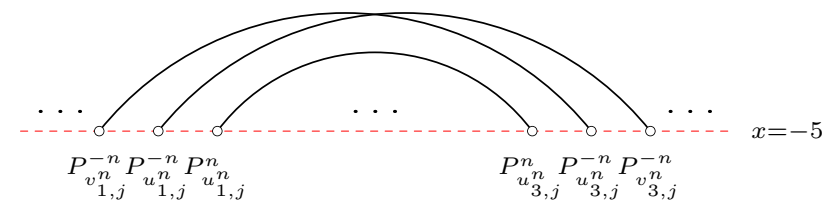

(1)

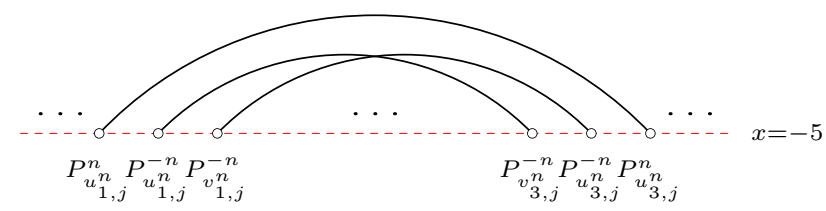

(2)

Figure 3.3: An auxiliary graph for the drawing of $\mathrm{Out}_{\mathbb{R}_{-5}^{5} \times \mathbb{R}_{-5}^{5}}\left(E_{\text {blue }}^{n}\right)$

By Inductive rule for the drawing of $E_{b l u e}^{n}$ and Observation 2.1, we have that

$$
\begin{gathered}
\nu_{\Gamma_{n}}\left(\operatorname{In}\left(\bigcup_{t \in\{-n, n\}} \mathscr{I}_{t}\left(U_{1,1}^{n}\right)\right)\right)=0, \\
\nu_{\Gamma_{n}}\left(\operatorname{In}\left(\bigcup_{t \in\{-n, n\}} \mathscr{I}_{t}\left(U_{1,1}^{n}\right)\right), \operatorname{In}\left(\bigcup_{t \in\{-n, n\}} \mathscr{I}_{t}\left(V_{1,1}^{n}\right)\right)\right)=0,
\end{gathered}
$$

and that

$$
\nu_{\Gamma_{n}}\left(\operatorname{In}\left(\bigcup_{t \in\{-n, n\}} \mathscr{I}_{t}\left(V_{1,1}^{n}\right)\right)\right)=\left(\begin{array}{c}
2^{n-6} \\
2
\end{array}\right)+\left(\begin{array}{c}
2^{n-7} \\
2
\end{array}\right)+2 \cdot\left(\begin{array}{c}
2^{n-8} \\
2
\end{array}\right)
$$

where

$\left(\begin{array}{c}2^{n-6} \\ 2\end{array}\right)$ crossings are produced between $\left\{\operatorname{In}\left(\mathscr{I}_{n}\left(v_{1, j}^{n}\right)\right): j \in\left[2^{n-4}-2^{n-6}+1,2^{n-4}\right]\right\}$ and $\left\{\operatorname{In}\left(\mathscr{I}_{-n}\left(v_{1, j}^{n}\right)\right): j \in\right.$ $\left.\left[2^{n-4}-2^{n-6}+1,2^{n-4}\right]\right\}$

$\left(\begin{array}{c}2^{n-7} \\ 2\end{array}\right)$ crossings are produced between $\left\{\operatorname{In}\left(\mathscr{I}_{n}\left(v_{1, j}^{n}\right)\right): j \in\left[2^{n-5}+1,2^{n-5}+2^{n-7}\right]\right\}$ and $\left\{\operatorname{In}\left(\mathscr{I}_{-n}\left(v_{1, j}^{n}\right)\right): j \in\right.$ $\left.\left[2^{n-5}+1,2^{n-5}+2^{n-7}\right]\right\}$

$2 \cdot\left(\begin{array}{c}2^{n-8} \\ 2\end{array}\right)$ crossings are produced between $\left\{\operatorname{In}\left(\mathscr{I}_{n}\left(v_{1, j}^{n}\right)\right): j \in\left[2^{n-5}+2^{n-7}+1,2^{n-5}+2^{n-6}\right]\right\}$ and $\left\{\operatorname{In}\left(\mathscr{I}_{-n}\left(v_{1, j}^{n}\right)\right):\right.$ $\left.j \in\left[2^{n-5}+2^{n-7}+1,2^{n-5}+2^{n-6}\right]\right\}$.

Combined (70), (71) and (72), we have

$$
\nu_{\Gamma_{n}}\left(\operatorname{In}\left(\bigcup_{t \in\{-n, n\}} \mathscr{I}_{t}\left(U_{1,1}^{n} \cup V_{1,1}^{n}\right)\right)\right)=\left(\begin{array}{c}
2^{n-6} \\
2
\end{array}\right)+\left(\begin{array}{c}
2^{n-7} \\
2
\end{array}\right)+2 \cdot\left(\begin{array}{c}
2^{n-8} \\
2
\end{array}\right) .
$$

Therefore, it follows from (64), (69) and (73) that

$$
\nu_{\Gamma_{n}}\left(E_{\text {blue }}^{n}\right)=2 \cdot 2^{n-3}+8 \cdot\left(\left(\begin{array}{c}
2^{n-6} \\
2
\end{array}\right)+\left(\begin{array}{c}
2^{n-7} \\
2
\end{array}\right)+2 \cdot\left(\begin{array}{c}
2^{n-8} \\
2
\end{array}\right)\right)=11 \cdot 2^{2 n-13}+2^{n-3} .
$$

2. Calculation of $\nu_{\Gamma_{n}}\left(E_{r e d}^{n}\right)$.

By (27), (41), (42), Inductive rule for the drawing of $E_{\text {red }}^{n}$ and Property B, we have that

$$
\nu_{\Gamma_{n}}\left(E_{\text {red }}^{n}\right)=8 \cdot \nu_{\Gamma_{n}}\left(\operatorname{In}\left(\bigcup_{t \in\{1,2\}} \mathscr{I}_{t}\left(U_{1,1}^{n} \cup V_{1,1}^{n}\right)\right)\right)+2 \cdot \nu_{\Gamma_{n}}\left(\mathscr{I}_{2}\left(U_{1,1}^{n} \cup V_{1,1}^{n}\right), \mathscr{I}_{2}\left(U_{1,2}^{n} \cup V_{1,2}^{n}\right)\right) .
$$

Now we calculate $\nu_{\Gamma_{n}}\left(\operatorname{In}\left(\bigcup_{t \in\{1,2\}} \mathscr{I}_{t}\left(U_{1,1}^{n} \cup V_{1,1}^{n}\right)\right)\right)$. 
By Inductive rule for the drawing of $E_{r e d}^{n}$ and Observation 2.1. we have that

$$
\nu_{\Gamma_{n}}\left(\operatorname{In}\left(\bigcup_{t \in\{1,2\}} \mathscr{I}_{t}\left(U_{1,1}^{n}\right)\right)\right)=\left(\begin{array}{c}
2^{n-6} \\
2
\end{array}\right)+3 \cdot 2^{n-6} \cdot 2^{n-6}
$$

where

$\left(\begin{array}{c}2^{n-6} \\ 2\end{array}\right)$ crossings are produced between $\left\{\operatorname{In}\left(\mathscr{I}_{1}\left(u_{1, j}^{n}\right)\right): j \in\left[2^{n-4}-2^{n-6}+1,2^{n-4}\right]\right\}$ and $\left\{\operatorname{In}\left(\mathscr{I}_{2}\left(u_{1, j}^{n}\right)\right): j \in\right.$ $\left.\left[2^{n-4}-2^{n-6}+1,2^{n-4}\right]\right\}$

$3 \cdot 2^{n-6} \cdot 2^{n-6}$ crossings are produced between $\left\{\operatorname{In}\left(\mathscr{I}_{1}\left(u_{1, j}^{n}\right)\right): j \in\left[2^{n-4}-2^{n-6}+1,2^{n-4}\right]\right\}$ and $\left\{\operatorname{In}\left(\mathscr{I}_{2}\left(u_{1, j}^{n}\right)\right):\right.$ $\left.j \in\left[1,2^{n-4}-2^{n-6}\right]\right\}$,

and that

$$
\nu_{\Gamma_{n}}\left(\operatorname{In}\left(\bigcup_{t \in\{1,2\}} \mathscr{I}_{t}\left(V_{1,1}^{n}\right)\right)\right)=\left(\begin{array}{c}
2^{n-4} \\
2
\end{array}\right)
$$

where $\left(\begin{array}{c}2^{n-4} \\ 2\end{array}\right)$ crossings are produced between $\left\{\operatorname{In}\left(\mathscr{I}_{1}\left(v_{1, j}^{n}\right)\right): j \in\left[1,2^{n-4}\right]\right\}$ and $\left\{\operatorname{In}\left(\mathscr{I}_{2}\left(v_{1, j}^{n}\right)\right): j \in\left[1,2^{n-4}\right]\right\}$,

and that

$$
\nu_{\Gamma_{n}}\left(\operatorname{In}\left(\bigcup_{t \in\{1,2\}} \mathscr{I}_{t}\left(U_{1,1}^{n}\right)\right), \operatorname{In}\left(\bigcup_{t \in\{1,2\}} \mathscr{I}_{t}\left(V_{1,1}^{n}\right)\right)\right)=3 \cdot 2^{n-7} \cdot 2^{n-4}+2^{n-6} \cdot 2^{n-5}
$$

where

$3 \cdot 2^{n-7} \cdot 2^{n-4}$ crossings are produced between $\left\{\operatorname{In}\left(\mathscr{I}_{2}\left(u_{1, j}^{n}\right)\right): j \in\left[1,2^{n-6}+2^{n-7}\right]\right\}$ and $\left\{\operatorname{In}\left(\mathscr{I}_{1}\left(v_{1, j}^{n}\right)\right): j \in\right.$ $\left.\left[1,2^{n-4}\right]\right\}$,

$2^{n-6} \cdot 2^{n-5}$ crossings are produced between $\left\{\operatorname{In}\left(\mathscr{I}_{2}\left(u_{1, j}^{n}\right)\right): j \in\left[2^{n-6}+2^{n-7}+1,2^{n-5}+2^{n-7}\right]\right\}$ and $\left\{\operatorname{In}\left(\mathscr{I}_{1}\left(v_{1, j}^{n}\right)\right): j \in\left[2^{n-5}+1,2^{n-4}\right]\right\}$.

Combined (76), (77) and (78), we have

$$
\begin{aligned}
& \nu_{\Gamma_{n}}\left(\operatorname{In}\left(\bigcup_{t \in\{1,2\}} \mathscr{I}_{t}\left(U_{1,1}^{n} \cup V_{1,1}^{n}\right)\right)\right) \\
= & \left(\left(\begin{array}{c}
2^{n-6} \\
2
\end{array}\right)+3 \cdot 2^{n-6} \cdot 2^{n-6}\right)+\left(\begin{array}{c}
2^{n-4} \\
2
\end{array}\right)+\left(3 \cdot 2^{n-7} \cdot 2^{n-4}+2^{n-6} \cdot 2^{n-5}\right) \\
= & 39 \cdot 2^{2 n-13}-5 \cdot 2^{n-7} .
\end{aligned}
$$

On the other hand, it is easy to see that

$$
\nu_{\Gamma_{n}}\left(\mathscr{I}_{2}\left(U_{1,1}^{n} \cup V_{1,1}^{n}\right), \mathscr{I}_{2}\left(U_{1,2}^{n} \cup V_{1,2}^{n}\right)\right)=2^{n-3} \cdot 2^{n-3}=4^{n-3} .
$$

Therefore, it follows from (75), (779) and (80) that

$$
\nu_{\Gamma_{n}}\left(E_{r e d}^{n}\right)=8 \cdot\left(39 \cdot 2^{2 n-13}-5 \cdot 2^{n-7}\right)+2 \cdot 4^{n-3}=71 \cdot 2^{2 n-10}-5 \cdot 2^{n-4} .
$$

3. Calculation of $\nu_{\Gamma_{n}}\left(E_{\text {black }}^{n}\right)$.

By Property B and Lemma 2.1, we have

$$
\begin{aligned}
\nu_{\Gamma_{n}}\left(E_{\text {black }}^{n}\right)=4 \cdot & \nu_{\Gamma_{n}}\left(E\left(U_{1}^{n} \cup V_{1}^{n}\right)\right) \\
=4 \cdot & \left(\nu_{\Gamma_{n}}\left(E\left(U_{1}^{n}\right)\right)+\nu_{\Gamma_{n}}\left(E\left(V_{1}^{n}\right)\right)+\nu_{\Gamma_{n}}\left(E\left[U_{1}^{n}, V_{1}^{n}\right]\right)\right. \\
& \left.\quad+\nu_{\Gamma_{n}}\left(E\left[U_{1}^{n}, V_{1}^{n}\right], E\left(U_{1}^{n}\right)\right)+\nu_{\Gamma_{n}}\left(E\left[U_{1}^{n}, V_{1}^{n}\right], E\left(V_{1}^{n}\right)\right)\right) .
\end{aligned}
$$


By Lemma 2.10, we have

$$
\begin{aligned}
& \nu_{\Gamma_{n}}\left(E\left(U_{1}^{n}\right)\right)=\nu_{\Gamma_{n}}\left(E\left(V_{1}^{n}\right)\right) \\
= & \nu_{\Upsilon_{(n-5)}}\left(\mathcal{E}^{(n-5)}\right) \\
= & \frac{194}{3} \cdot 4^{n-6}-\left(4 \cdot(n-5)^{2}+23 \cdot(n-5)+\frac{101}{3}-\frac{7 \cdot\left(1+(-1)^{n-5}\right)}{6}\right) \cdot 2^{n-6} \\
= & \frac{97}{3} \cdot 2^{2 n-11}-\left(4 n^{2}-17 n+\frac{56}{3}-\frac{7 \cdot\left(1+(-1)^{n-1}\right)}{6}\right) \cdot 2^{n-6} .
\end{aligned}
$$

By (31), (32), (33), (36), (37) and Conclusion 4 in Lemma 2.2, we have

$$
\nu_{\Gamma_{n}}\left(E\left[U_{1}^{n}, V_{1}^{n}\right]\right)=2^{n-4}
$$

and by (30) and Lemma 2.6, we have

$$
\begin{aligned}
& \nu_{\Gamma_{n}}\left(E\left[U_{1}^{n}, V_{1}^{n}\right], E\left(U_{1}^{n}\right)\right)=\nu_{\Gamma_{n}}\left(E\left[U_{1}^{n}, V_{1}^{n}\right], E\left(V_{1}^{n}\right)\right) \\
= & 2 \cdot \mathcal{C}_{-}^{(n-5)} \\
= & 2 \cdot\left(\frac{5}{3} \cdot 4^{n-4}-\left(2 \cdot(n-5)+\frac{13}{3}+\frac{7 \cdot\left(1+(-1)^{n-5}\right)}{6}\right) \cdot 2^{n-5}\right) \\
= & \frac{5}{3} \cdot 2^{2 n-7}-\left(2 n-\frac{17}{3}+\frac{7 \cdot\left(1+(-1)^{n-1}\right)}{6}\right) \cdot 2^{n-4} \cdot
\end{aligned}
$$

Therefore, it follows from (82), (83), (84) and (85) that

$$
\begin{aligned}
\nu_{\Gamma_{n}}\left(E_{\text {black }}^{n}\right)= & 4 \cdot\left(\frac{97}{3} \cdot 2^{2 n-10}-\left(4 n^{2}-17 n+\frac{56}{3}-\frac{7 \cdot\left(1+(-1)^{n-1}\right)}{6}\right) \cdot 2^{n-5}+2^{n-4}\right. \\
& \left.\quad+\frac{5}{3} \cdot 2^{2 n-6}-\left(2 n-\frac{17}{3}+\frac{7 \cdot\left(1+(-1)^{n-1}\right)}{6}\right) \cdot 2^{n-3}\right) \\
= & 59 \cdot 2^{2 n-8}-\left(4 n^{2}-9 n-6\right) \cdot 2^{n-3}-7 \cdot\left(1+(-1)^{n-1}\right) \cdot 2^{n-4} .
\end{aligned}
$$

4. Calculation of $\nu_{\Gamma_{n}}\left(E_{\text {red }}^{n}, E_{\text {black }}^{n}\right)$.

By Property B and Lemma 2.1. we have that

$$
\begin{aligned}
& \nu_{\Gamma_{n}}\left(E_{\text {red }}^{n}, E_{\text {black }}^{n}\right) \\
= & 8 \cdot \nu_{\Gamma_{n}}\left(\operatorname{In}\left(E_{\text {red }}^{n}\right), \operatorname{In}\left(E_{\text {black }}^{n}\right)\right) \\
= & 8 \cdot \nu_{\Gamma_{n}}\left(\operatorname{In}\left(\bigcup_{t \in\{1,2\}} \mathscr{I}_{t}\left(U_{1,1}^{n} \cup V_{1,1}^{n}\right)\right), \operatorname{In}\left(E\left(U_{1}^{n} \cup V_{1}^{n}\right)\right)\right) \\
= & 8 \cdot\left(\nu_{\Gamma_{n}}\left(\operatorname{In}\left(\bigcup_{t \in\{1,2\}} \mathscr{I}_{t}\left(U_{1,1}^{n}\right)\right), \operatorname{In}\left(E\left(U_{1}^{n}\right)\right)\right)+\nu_{\Gamma_{n}}\left(\operatorname{In}\left(\bigcup_{t \in\{1,2\}} \mathscr{I}_{t}\left(U_{1,1}^{n}\right)\right), E\left[U_{1,1}^{n}, V_{1,1}^{n}\right]\right)\right. \\
& \quad+\nu_{\Gamma_{n}}\left(\operatorname{In}\left(\bigcup_{t \in\{1,2\}} \mathscr{I}_{t}\left(U_{1,1}^{n}\right)\right), \operatorname{In}\left(E\left(V_{1}^{n}\right)\right)\right)+\nu_{\Gamma_{n}}\left(\operatorname{In}\left(\bigcup_{t \in\{1,2\}} \mathscr{I}_{t}\left(V_{1,1}^{n}\right)\right), \operatorname{In}\left(E\left(U_{1}^{n}\right)\right)\right) \\
& \left.\quad+\nu_{\Gamma_{n}}\left(\operatorname{In}\left(\bigcup_{t \in\{1,2\}} \mathscr{I}_{t}\left(V_{1,1}^{n}\right)\right), E\left[U_{1,1}^{n}, V_{1,1}^{n}\right]\right)+\nu_{\Gamma_{n}}\left(\operatorname{In}\left(\bigcup_{t \in\{1,2\}} \mathscr{I}_{t}\left(V_{1,1}^{n}\right)\right), \operatorname{In}\left(E\left(V_{1}^{n}\right)\right)\right)\right)
\end{aligned}
$$

By Observation 2.1] Lemma 2.6. Lemma 2.7 and Inductive rule for the drawing of $E_{r e d}^{n}$, we have that

$$
\begin{aligned}
& \nu_{\Gamma_{n}}\left(\operatorname{In}\left(\bigcup_{t \in\{1,2\}} \mathscr{I}_{t}\left(U_{1,1}^{n}\right)\right), \operatorname{In}\left(E\left(U_{1}^{n}\right)\right)\right) \\
= & \sum_{j \in \mathcal{I}_{6}^{(n-5)} \cup \mathcal{I}_{7}^{(n-5)} \cup \mathcal{I}_{8}^{(n-5)}}\left(\mathscr{C}_{+}^{(n-5)}\left(\mathcal{E}^{(n-5)}, z_{j}^{(n-5)}\right)+\mathscr{C}_{-}^{(n-5)}\left(\mathcal{E}^{(n-5)}, z_{j}^{(n-5)}\right)\right) \\
& +2 \sum_{j \in \mathcal{I}_{5}^{(n-5)}} \mathscr{C}_{-}^{(n-5)}\left(\mathcal{E}^{(n-5)}, z_{j}^{(n-5)}\right) \\
= & \frac{335}{3} \cdot 4^{n-7}-\left(16 n-\frac{100}{3}+\frac{7 \cdot\left(1+(-1)^{n-1}\right)}{3}\right) \cdot 2^{n-8},
\end{aligned}
$$


and that

$$
\nu_{\Gamma_{n}}\left(\operatorname{In}\left(\bigcup_{t \in\{1,2\}} \mathscr{I}_{t}\left(U_{1,1}^{n}\right)\right), E\left[U_{1,1}^{n}, V_{1,1}^{n}\right]\right)=2 \cdot 2 \cdot\left(\begin{array}{c}
3 \cdot 2^{n-7} \\
2
\end{array}\right)+2 \cdot 2 \cdot\left(\begin{array}{c}
2^{n-7} \\
2
\end{array}\right)+2 \cdot\left(\begin{array}{c}
2^{n-6} \\
2
\end{array}\right)+2 \cdot 3 \cdot 2^{n-6} \cdot 2^{n-6}
$$

where

$2 \cdot 2 \cdot\left(\begin{array}{c}3 \cdot 2^{n-7} \\ 2\end{array}\right)$ crossings are produced between $\left\{\operatorname{In}\left(\mathscr{I}_{2}\left(u_{1, j}^{n}\right)\right): j \in\left[1,2^{n-6}+2^{n-7}\right] \cup\left[2^{n-5}+2^{n-7}+1,2^{n-4}\right]\right\}$ and $E\left[U_{1,1}^{n}, V_{1,1}^{n}\right]$;

$2 \cdot 2 \cdot\left(\begin{array}{c}2^{n-7} \\ 2\end{array}\right)$ crossings are produced between $\left\{\operatorname{In}\left(\mathscr{I}_{2}\left(u_{1, j}^{n}\right)\right): j \in\left[2^{n-6}+2^{n-7}+1,2^{n-5}+2^{n-7}\right]\right\}$ and $E\left[U_{1,1}^{n}, V_{1,1}^{n}\right]$;

$2 \cdot\left(\begin{array}{c}2^{n-6} \\ 2\end{array}\right)+2 \cdot 3 \cdot 2^{n-6} \cdot 2^{n-6}$ crossings are produced between $\left\{\operatorname{In}\left(\mathscr{I}_{1}\left(u_{1, j}^{n}\right)\right): j \in\left[2^{n-5}+2^{n-6}+1,2^{n-4}\right]\right\}$ and $E\left[U_{1,1}^{n}, V_{1,1}^{n}\right]$,

and that

$$
\nu_{\Gamma_{n}}\left(\operatorname{In}\left(\bigcup_{t \in\{1,2\}} \mathscr{I}_{t}\left(U_{1,1}^{n}\right)\right), \operatorname{In}\left(E\left(V_{1}^{n}\right)\right)\right)=4 \cdot 2^{n-5} \cdot 2^{n-6}+2 \cdot 2^{n-4} \cdot 3 \cdot 2^{n-7}
$$

where

$4 \cdot 2^{n-5} \cdot 2^{n-6}$ crossings are produced between $\left\{\operatorname{In}\left(\mathscr{I}_{2}\left(u_{1, j}^{n}\right)\right): j \in\left[2^{n-6}+2^{n-7}+1,2^{n-5}+2^{n-7}\right]\right\}$ and $\operatorname{In}\left(E\left(V_{1}^{n}\right)\right)$

$2 \cdot 2^{n-4} \cdot 3 \cdot 2^{n-7}$ crossings are produced between $\left\{\operatorname{In}\left(\mathscr{I}_{2}\left(u_{1, j}^{n}\right)\right): j \in\left[2^{n-5}+2^{n-7}+1,2^{n-4}\right]\right\}$ and $\operatorname{In}\left(E\left(V_{1}^{n}\right)\right)$, and that

$\nu_{\Gamma_{n}}\left(\operatorname{In}\left(\bigcup_{t \in\{1,2\}} \mathscr{I}_{t}\left(V_{1,1}^{n}\right)\right), \operatorname{In}\left(E\left(V_{1}^{n}\right)\right)\right)=2 \cdot \frac{1}{2} \cdot \mathcal{C}_{+}^{(n-5)}=\frac{7}{3} \cdot 4^{n-4}-\left(2 n-\frac{14}{3}+\frac{7\left(1+(-1)^{n}\right)}{6}\right) \cdot 2^{n-5}$,

and that

$$
\nu_{\Gamma_{n}}\left(\operatorname{In}\left(\bigcup_{t \in\{1,2\}} \mathscr{I}_{t}\left(V_{1,1}^{n}\right)\right), \operatorname{In}\left(E\left(U_{1}^{n}\right)\right)\right)=0
$$

and that

$$
\nu_{\Gamma_{n}}\left(\operatorname{In}\left(\bigcup_{t \in\{1,2\}} \mathscr{I}_{t}\left(V_{1,1}^{n}\right)\right), E\left[U_{1,1}^{n}, V_{1,1}^{n}\right]\right)=0 .
$$

Therefore, it follows from (87), (88), (89), (90), (91), (92) and (93) that

$$
\begin{aligned}
\nu_{\Gamma_{n}}\left(E_{\text {red }}^{n}, E_{\text {black }}^{n}\right) & =8 \cdot\left(389 \cdot 2^{2 n-14}-(n-1) \cdot 2^{n-3}+7 \cdot\left(1+(-1)^{n-1}\right) \cdot 2^{n-8}\right) \\
& =389 \cdot 2^{2 n-11}-(n-1) \cdot 2^{n}+7 \cdot\left(1+(-1)^{n-1}\right) \cdot 2^{n-5} .
\end{aligned}
$$

5. Calculation of $\nu_{\Gamma_{n}}\left(E_{\text {blue }}^{n}, E_{r e d}^{n}\right)$.

By (27), Property B and Lemma 2.1, we have that

$$
\begin{aligned}
& \nu_{\Gamma_{n}}\left(E_{\text {blue }}^{n}, E_{\text {red }}^{n}\right) \\
= & 8 \cdot \nu_{\Gamma_{n}}\left(\operatorname{In}\left(E_{\text {blue }}^{n}\right), \operatorname{In}\left(E_{\text {red }}^{n}\right)\right) \\
= & 8 \cdot \nu_{\Gamma_{n}}\left(\operatorname{In}\left(\bigcup_{t \in\{-n, n\}} \mathscr{I}_{t}\left(U_{1,1}^{n} \cup V_{1,1}^{n}\right)\right), \operatorname{In}\left(\bigcup_{t \in\{1,2\}} \mathscr{I}_{t}\left(U_{1,1}^{n} \cup V_{1,1}^{n}\right)\right)\right) \\
= & 8 \cdot \nu_{\Gamma_{n}}\left(\operatorname{In}\left(\bigcup_{t \in\{-n, n\}} \mathscr{I}_{t}\left(U_{1,1}^{n}\right)\right) \cup \operatorname{In}\left(\bigcup_{t \in\{-n, n\}} \mathscr{I}_{t}\left(V_{1,1}^{n}\right)\right), \operatorname{In}\left(\bigcup_{t \in\{1,2\}} \mathscr{I}_{t}\left(U_{1,1}^{n}\right)\right) \cup \operatorname{In}\left(\bigcup_{t \in\{1,2\}} \mathscr{I}_{t}\left(V_{1,1}^{n}\right)\right)\right) \\
= & 8 \cdot\left(\nu_{\Gamma_{n}}\left(\operatorname{In}\left(\bigcup_{t \in\{-n, n\}} \mathscr{I}_{t}\left(U_{1,1}^{n}\right)\right), \operatorname{In}\left(\bigcup_{t \in\{1,2\}} \mathscr{I}_{t}\left(U_{1,1}^{n}\right)\right)\right)+\nu_{\Gamma_{n}}\left(\operatorname{In}\left(\bigcup_{t \in\{-n, n\}} \mathscr{I}_{t}\left(U_{1,1}^{n}\right)\right), \operatorname{In}\left(\bigcup_{t \in\{1,2\}} \mathscr{I}_{t}\left(V_{1,1}^{n}\right)\right)\right)\right. \\
& \left.\quad+\nu_{\Gamma_{n}}\left(\operatorname{In}\left(\bigcup_{t \in\{-n, n\}} \mathscr{I}_{t}\left(V_{1,1}^{n}\right)\right), \operatorname{In}\left(\bigcup_{t \in\{1,2\}} \mathscr{I}_{t}\left(U_{1,1}^{n}\right)\right)\right)+\nu_{\Gamma_{n}}\left(\operatorname{In}\left(\bigcup_{t \in\{-n, n\}} \mathscr{I}_{t}\left(V_{1,1}^{n}\right)\right), \operatorname{In}\left(\bigcup_{t \in\{1,2\}} \mathscr{I}_{t}\left(V_{1,1}^{n}\right)\right)\right)\right)
\end{aligned}
$$


By Inductive rule for the drawing of $E_{r e d}^{n}$, Inductive rule for the drawing of $E_{b l u e}^{n}$ and Observation 2.1, we have that

$$
\nu_{\Gamma_{n}}\left(\operatorname{In}\left(\bigcup_{t \in\{-n, n\}} \mathscr{I}_{t}\left(U_{1,1}^{n}\right)\right), \operatorname{In}\left(\bigcup_{t \in\{1,2\}} \mathscr{I}_{t}\left(V_{1,1}^{n}\right)\right)\right)=0
$$

and that

$$
\nu_{\Gamma_{n}}\left(\operatorname{In}\left(\bigcup_{t \in\{-n, n\}} \mathscr{I}_{t}\left(U_{1,1}^{n}\right)\right), \operatorname{In}\left(\bigcup_{t \in\{1,2\}} \mathscr{I}_{t}\left(U_{1,1}^{n}\right)\right)\right)=2 \cdot\left(\begin{array}{c}
2^{n-5}+2^{n-6} \\
2
\end{array}\right)
$$

where $2 \cdot\left(\begin{array}{c}2^{n-5}+2^{n-6} \\ 2\end{array}\right)$ crossings are produced between $\operatorname{In}\left(\bigcup_{t \in\{-n, n\}} \mathscr{I}_{t}\left(U_{1,1}^{n}\right)\right)$ and $\left\{\operatorname{In}\left(\mathscr{I}_{1}\left(u_{1, j}^{n}\right)\right): j \in\left[1,2^{n-5}+\right.\right.$ $\left.\left.2^{n-6}\right]\right\}$

and that

$$
\nu_{\Gamma_{n}}\left(\operatorname{In}\left(\bigcup_{t \in\{-n, n\}} \mathscr{I}_{t}\left(V_{1,1}^{n}\right)\right), \operatorname{In}\left(\bigcup_{t \in\{1,2\}} \mathscr{I}_{t}\left(V_{1,1}^{n}\right)\right)\right)=2 \cdot\left(\begin{array}{c}
2^{n-5} \\
2
\end{array}\right)
$$

where $2 \cdot\left(\begin{array}{c}2^{n-5} \\ 2\end{array}\right)$ crossings are produced between $\left\{\operatorname{In}\left(\mathscr{I}_{n}\left(v_{1, j}^{n}\right)\right): j \in\left[1,2^{n-5}\right]\right\}$ and $\left\{\operatorname{In}\left(\bigcup_{t \in\{1,2\}} \mathscr{I}_{t}\left(v_{1, j}^{n}\right)\right): j \in\right.$ $\left.\left[1,2^{n-5}\right]\right\}$.

It remains to calculate $\nu_{\Gamma_{n}}\left(\operatorname{In}\left(\bigcup_{t \in\{-n, n\}} \mathscr{I}_{t}\left(V_{1,1}^{n}\right)\right), \operatorname{In}\left(\bigcup_{t \in\{1,2\}} \mathscr{I}_{t}\left(U_{1,1}^{n}\right)\right)\right)$.

By Lemma 3.1. we have that

$$
s_{n, j \times 2^{n-7}+1}=2 \cdot s_{n-1, j \times 2^{n-8}+1}=2^{2} \cdot s_{n-2, j \times 2^{n-9}+1}=\cdots=2^{n-8} \cdot s_{8,2 j+1}
$$

for all $j \in\left[0,2^{3}\right]$,

and that

$$
\begin{aligned}
s_{n, j \times 2^{n-7}} & =s_{n-1, j \times 2^{n-8}}+s_{n-1, j \times 2^{n-8}+1} \\
& =s_{n-2, j \times 2^{n-9}}+s_{n-2, j \times 2^{n-9}+1}+2 \cdot s_{n-2, j \times 2^{n-9}+1} \\
& =s_{n-2, j \times 2^{n-9}}+\left(2^{2}-1\right) \cdot s_{n-2, j \times 2^{n-9}+1} \\
& =s_{n-3, j \times 2^{n-10}}+\left(2^{3}-1\right) \cdot s_{n-3, j \times 2^{n-10}+1} \\
& \vdots \\
& =s_{8,2 j}+\left(2^{n-8}-1\right) \cdot s_{8,2 j+1}
\end{aligned}
$$

for all $j \in\left[1,2^{3}\right]$.

By (99) and Lemma 3.1 we have that

$$
\begin{aligned}
s_{n, j \times 2^{n-7}+2} & =s_{n, 2 \cdot\left(j \times 2^{n-8}+1\right)} \\
& =s_{n-1, j \times 2^{n-8}+1}+s_{n-1, j \times 2^{n-8}+2} \\
& =2 \cdot s_{n-2, j \times 2^{n-9}+1}+s_{n-2, j \times 2^{n-9}+1}+s_{n-2, j \times 2^{n-9}+2} \\
& =\left(2^{2}-1\right) \cdot s_{n-2, j \times 2^{n-9}+1}+s_{n-2, j \times 2^{n-9}+2} \\
& =\left(2^{3}-1\right) \cdot s_{n-3, j \times 2^{n-10}+1}+s_{n-3, j \times 2^{n-10}+2} \\
& \vdots \\
& =\left(2^{n-8}-1\right) \cdot s_{8,2 j+1}+s_{8,2 j+2}
\end{aligned}
$$

for all $j \in\left[1,2^{3}-1\right]$. 
By (99), (100) and Lemma 3.1] we have that

$$
\begin{aligned}
\sum_{j=1}^{2^{n-4}+1} s_{n, i} & =2 \sum_{j=1}^{2^{n-5}+1} s_{n-1, j}+\sum_{j=1}^{2^{n-5}}\left(s_{n-1, j}+s_{n-1, j+1}\right) \\
& =4 \sum_{j=1}^{2^{n-5}+1} s_{n-1, j}-s_{n-1,2^{n-5}+1}-s_{n-1,1} \\
& =4 \sum_{j=1}^{2^{n-5}+1} s_{n-1, j}-2^{n-9} \cdot s_{8,17}-2^{n-9} \cdot s_{8,1} \\
& =4 \sum_{j=1}^{2^{n-5}+1} s_{n-1, j}-\left(s_{8,17}+s_{8,1}\right) \cdot 2^{n-9} \\
& =4^{2} \sum_{j=1}^{2^{n-6}+1} s_{n-2, j}-\left(s_{8,17}+s_{8,1}\right) \sum_{j=0}^{1} 2^{n-9+j} \\
& \vdots \\
& =4^{n-8} \sum_{j=1}^{2^{4}+1} s_{8, j}-\left(s_{8,17}+s_{8,1}\right) \sum_{j=0}^{n-9} 2^{n-9+j} \\
& =4^{n-8} \sum_{j=1}^{2^{4}+1} s_{8, j}-7 \cdot 2^{2 n-15}+7 \cdot 2^{n-7} .
\end{aligned}
$$

It is easy to verify the following

\begin{tabular}{|c|c|c|c|c|c|c|c|c|c|c|c|c|c|c|c|c|}
\hline$s_{8,1}$ & $s_{8,2}$ & $s_{8,3}$ & $s_{8,4}$ & $s_{8,5}$ & $s_{8,6}$ & $s_{8,7}$ & $s_{8,8}$ & $s_{8,9}$ & $s_{8,10}$ & $s_{8,11}$ & $s_{8,12}$ & $s_{8,13}$ & $s_{8,14}$ & $s_{8,15}$ & $s_{8,16}$ & $s_{8,17}$ \\
\hline 22 & 20 & 18 & 16 & 14 & 12 & 10 & 10 & 10 & 8 & 6 & 6 & 6 & 6 & 6 & 6 & 6 \\
\hline
\end{tabular}

Table 3.1: The values of $s_{8, j}$ for $j \in\left[1,2^{4}+1\right]$ 
By (99), (100), (102), (46), (49), Property A, Assertion C, Lemma 3.1 and Table 3.1, we conclude that

$$
\begin{aligned}
& \nu_{\Gamma_{n}}\left(\operatorname{In}\left(\bigcup_{t \in\{-n, n\}} \mathscr{I}_{t}\left(V_{1,1}^{n}\right)\right), \operatorname{In}\left(\bigcup_{t \in\{1,2\}} \mathscr{I}_{t}\left(U_{1,1}^{n}\right)\right)\right) \\
&= \nu_{\Gamma_{n}}\left(\operatorname{In}\left(\mathscr{I}_{-n}\left(V_{1,1}^{n}\right)\right), \operatorname{In}\left(\bigcup_{t \in\{1,2\}} \mathscr{I}_{t}\left(U_{1,1}^{n}\right)\right)\right)+\left(\operatorname{In}\left(\mathscr{I}_{n}\left(V_{1,1}^{n}\right)\right), \operatorname{In}\left(\bigcup_{t \in\{1,2\}} \mathscr{I}_{t}\left(U_{1,1}^{n}\right)\right)\right) \\
&=\left(\sum_{j=1}^{2^{n-4}} s_{n, j}+\left(s_{n, 2^{n-6}+1}-s_{n, 2^{n-6}}\right)+\left(s_{n, 2^{n-6}+2^{n-7}+1}-s_{n, 2^{n-6}+2^{n-7}}\right)+\left(s_{n, 2^{n-5}+1}-s_{n, 2^{n-5}}\right)\right. \\
&+\left(s_{n, 2^{n-5}+2^{n-7}+1}-s_{n, 2^{n-5}+2^{n-7}}\right)+\left(s_{n, 2^{n-5}+2^{n-6}+1}-s_{n, 2^{n-5}+2^{n-6}}\right) \\
&\left.+\sum_{j=2^{n-5}+2^{n-6}+2}\left(s_{n, j+1}-s_{n, j}\right)\right) \\
&+\left(2^{n-5} \cdot s_{n, 1}+2^{n-7} \cdot s_{n, 2^{n-5}+1}+2^{n-8} \cdot s_{n, 2^{n-5}+2^{n-7}+1}+2^{n-8} \cdot s_{n, 2^{n-5}+2^{n-6}+1}+2^{n-6} \cdot s_{n, 2^{n-4}+1}\right) \\
&=\left(\sum^{n-4}+1\right. \\
&\left.\quad s_{n, j}+\sum_{j=2}^{6} s_{n, j \times 2^{n-7}+1}-\sum_{j=2}^{6} s_{n, j \times 2^{n-7}}-s_{n, 6 \times 2^{n-7}+2}\right) \\
&+\left(2^{n-5} \cdot s_{n, 1}+2^{n-7} \cdot s_{n, 4 \times 2^{n-7}+1}+2^{n-8} \cdot s_{n, 5 \times 2^{n-7}+1}+2^{n-8} \cdot s_{n, 6 \times 2^{n-7}+1}+2^{n-6} \cdot s_{n, 8 \times 2^{n-7}+1}\right) \\
&=\left(\left(4^{n-8} \sum_{j=1}^{2^{4}+1} s_{8, j}-7 \cdot 2^{2 n-15}+7 \cdot 2^{n-7}\right)+\sum_{j=2}^{6} 2^{n-8} \cdot s_{8,2 j+1}-\sum_{j=2}^{6}\left(s_{8,2 j}+\left(2^{n-8}-1\right) \cdot s_{8,2 j+1}\right)\right. \\
&\left.\quad-\left(\left(2^{n-8}-1\right) \cdot s_{8,13}+s_{8,14}\right)\right) \\
&+\left(2^{n-5} \cdot 2^{n-8} \cdot s_{8,1}+2^{n-7} \cdot 2^{n-8} \cdot s_{8,9}+2^{n-8} \cdot 2^{n-8} \cdot s_{8,11}+2^{n-8} \cdot 2^{n-8} \cdot s_{8,13}+2^{n-6} \cdot 2^{n-8} \cdot s_{8,17}\right) \\
&=\left(\left(182 \cdot 2^{2 n-16}-7 \cdot 2^{2 n-15}+7 \cdot 2^{n-7}\right)+46 \cdot 2^{n-8}-\left(52+46 \cdot\left(2^{n-8}-1\right)\right)-\left(6 \cdot\left(2^{n-8}-1\right)+6\right)\right) \\
&+\left(22 \cdot 2^{2 n-13}+10 \cdot 2^{2 n-15}+6 \cdot 2^{2 n-16}+6 \cdot 2^{2 n-16}+6 \cdot 2^{2 n-14}\right) \\
&= 25 \cdot 2^{2 n-12}+2^{n-5}-6 .
\end{aligned}
$$

Therefore, it follows from (95), (96), (97), (98) and (103) that

$$
\begin{aligned}
\nu_{\Gamma_{n}}\left(E_{\text {blue }}^{n}, E_{\text {red }}^{n}\right) & =8 \cdot\left(2 \cdot\left(\begin{array}{c}
3 \cdot 2^{n-6} \\
2
\end{array}\right)+0+2 \cdot\left(\begin{array}{c}
2^{n-5} \\
2
\end{array}\right)+\left(25 \cdot 2^{2 n-12}+2^{n-5}-6\right)\right) \\
& =19 \cdot 2^{2 n-8}-3 \cdot 2^{n-3}-48 .
\end{aligned}
$$

6. Calculation of $\nu_{\Gamma_{n}}\left(E_{\text {blue }}^{n}, E_{\text {black }}^{n}\right)$.

By Property B and Lemma 2.1] we have that

$$
\begin{aligned}
& \nu_{\Gamma_{n}}\left(E_{\text {blue }}^{n}, E_{\text {black }}^{n}\right) \\
= & 8 \cdot \nu_{\Gamma_{n}}\left(\operatorname{In}\left(E_{\text {blue }}^{n}\right), \operatorname{In}\left(E_{\text {black }}^{n}\right)\right) \\
= & 8 \cdot \nu_{\Gamma_{n}}\left(\operatorname{In}\left(\bigcup_{t \in\{-n, n\}} \mathscr{I}_{t}\left(U_{1,1}^{n} \cup V_{1,1}^{n}\right)\right), \operatorname{In}\left(E\left(U_{1}^{n} \cup V_{1}^{n}\right)\right)\right) \\
= & 8 \cdot\left(\nu_{\Gamma_{n}}\left(\operatorname{In}\left(\bigcup_{t \in\{-n, n\}} \mathscr{I}_{t}\left(U_{1,1}^{n}\right)\right), \operatorname{In}\left(E\left(U_{1}^{n} \cup V_{1}^{n}\right)\right)\right)+\nu_{\Gamma_{n}}\left(\operatorname{In}\left(\bigcup_{t \in\{-n, n\}} \mathscr{I}_{t}\left(V_{1,1}^{n}\right)\right), \operatorname{In}\left(E\left(U_{1}^{n} \cup V_{1}^{n}\right)\right)\right)\right) \\
= & 8 \cdot\left(\nu_{\Gamma_{n}}\left(\operatorname{In}\left(\bigcup_{t \in\{-n, n\}} \mathscr{I}_{t}\left(U_{1,1}^{n}\right)\right), \operatorname{In}\left(E\left(U_{1}^{n} \cup V_{1}^{n}\right)\right)\right)\right. \\
& \left.\quad+\nu_{\Gamma_{n}}\left(\operatorname{In}\left(\bigcup_{t \in\{-n, n\}} \mathscr{I}_{t}\left(V_{1,1}^{n}\right)\right), \operatorname{In}\left(E\left(U_{1}^{n}\right) \cup E\left[U_{1}^{n}, V_{1}^{n}\right]\right)\right)+\nu_{\Gamma_{n}}\left(\operatorname{In}\left(\bigcup_{t \in\{-n, n\}} \mathscr{I}_{t}\left(V_{1,1}^{n}\right)\right), \operatorname{In}\left(E\left(V_{1}^{n}\right)\right)\right)\right)
\end{aligned}
$$


By Lemma 2.6. Lemma 2.7 and Inductive rule for the drawing of $E_{b l u e}^{n}$, we have that

$$
\begin{aligned}
& \nu_{\Gamma_{n}}\left(\operatorname{In}\left(\bigcup_{t \in\{-n, n\}} \mathscr{I}_{t}\left(U_{1,1}^{n}\right)\right), \operatorname{In}\left(E\left(U_{1}^{n} \cup V_{1}^{n}\right)\right)\right) \\
= & \nu_{\Gamma_{n}}\left(\operatorname{In}\left(\bigcup_{t \in\{-n, n\}} \mathscr{I}_{t}\left(U_{1,1}^{n}\right)\right), \operatorname{In}\left(E\left(U_{1}^{n}\right)\right)\right) \\
= & 2 \cdot \frac{1}{2} \cdot \mathcal{C}_{+}^{(n-5)}=\frac{7}{3} \cdot 4^{n-4}-\left(2 n-\frac{14}{3}+\frac{7\left(1+(-1)^{n}\right)}{6}\right) \cdot 2^{n-5}
\end{aligned}
$$

and

$$
\begin{aligned}
& \nu_{\Gamma_{n}}\left(\operatorname{In}\left(\bigcup_{t \in\{-n, n\}} \mathscr{I}_{t}\left(V_{1,1}^{n}\right)\right), \operatorname{In}\left(E\left(V_{1}^{n}\right)\right)\right) \\
& =\nu_{\Gamma_{n}}\left(\operatorname{In}\left(\mathscr{I}_{-n}\left(V_{1,1}^{n}\right)\right), \operatorname{In}\left(E\left(V_{1}^{n}\right)\right)\right)+\nu_{\Gamma_{n}}\left(\operatorname{In}\left(\mathscr{I}_{n}\left(V_{1,1}^{n}\right)\right), \operatorname{In}\left(E\left(V_{1}^{n}\right)\right)\right) \\
& =\nu_{\Gamma_{n}}\left(\operatorname{In}\left(\mathscr{I}_{-n}\left(V_{1,1}^{n}\right)\right), \operatorname{In}\left(E\left(V_{1}^{n}\right)\right)\right)+\nu_{\Gamma_{n}}\left(\left\{\operatorname{In}\left(\mathscr{I}_{n}\left(v_{1, j}^{n}\right)\right): j \in\left[1,2^{n-5}\right]\right\}, \operatorname{In}\left(E\left(V_{1}^{n}\right)\right)\right) \\
& +\nu_{\Gamma_{n}}\left(\left\{\operatorname{In}\left(\mathscr{I}_{n}\left(v_{1, j}^{n}\right)\right): j \in\left[2^{n-5}+1,2^{n-4}\right]\right\}, \operatorname{In}\left(E\left(V_{1}^{n}\right)\right)\right) \\
& =\frac{1}{2} \cdot \mathcal{C}_{-}^{(n-5)}+\sum_{j \in \mathcal{I}_{1}^{(n-5)} \cup \mathcal{I}_{2}^{(n-5)}} \mathscr{C}_{+}^{(n-5)}\left(\mathcal{E}^{(n-5)}, z_{j}^{(n-5)}\right)+\sum_{j \in \mathcal{I}_{3}^{(n-5)} \cup \mathcal{I}_{4}^{(n-5)}} \mathscr{C}_{-}^{(n-5)}\left(\mathcal{E}^{(n-5)}, z_{j}^{(n-5)}\right) \\
& =\frac{5}{3} \cdot 2^{2 n-9}-\left(2 n-\frac{17}{3}+\frac{7 \cdot\left(1+(-1)^{n-1}\right)}{6}\right) \cdot 2^{n-6} \\
& +\frac{35}{3} \cdot 2^{2 n-13}-\left(4 n-\frac{28}{3}+\frac{7 \cdot\left(1+(-1)^{n}\right)}{3}\right) \cdot 2^{n-8} \\
& +\frac{43}{3} \cdot 2^{2 n-13}-\left(4 n-\frac{34}{3}+\frac{7 \cdot\left(1+(-1)^{n-1}\right)}{3}\right) \cdot 2^{n-8} \\
& =\frac{79}{3} \cdot 2^{2 n-12}-\left(4 n-\frac{29}{3}+\frac{7 \cdot\left(1+(-1)^{n-1}\right)}{6}\right) \cdot 2^{n-6} \text {. }
\end{aligned}
$$

It remains to calculate $\nu_{\Gamma_{n}}\left(\operatorname{In}\left(\bigcup_{t \in\{-n, n\}} \mathscr{I}_{t}\left(V_{1,1}^{n}\right)\right), \operatorname{In}\left(E\left(U_{1}^{n}\right) \cup E\left[U_{1}^{n}, V_{1}^{n}\right]\right)\right)$.

Similarly as (99), (100), (101) and (102), we can derive that

$$
t_{n, j \times 2^{n-7}+1}=2^{n-8} \cdot t_{8,2 j+1} \text { for all } j \in\left[0,2^{3}\right],
$$

and that

$$
t_{n, j \times 2^{n-7}}=t_{8,2 j}+\left(2^{n-8}-1\right) \cdot t_{8,2 j+1}+2 \cdot(n-8) \text { for all } j \in\left[1,2^{3}\right]
$$

and that

$$
t_{n, j \times 2^{n-7}+2}=\left(2^{n-8}-1\right) \cdot t_{8,2 j+1}+t_{8,2 j+2}+2 \cdot(n-8) \quad \text { for all } j \in\left[1,2^{3}-1\right]
$$

and that

$$
\sum_{j=1}^{2^{n-4}+1} t_{n, j}=4^{n-8} \sum_{j=1}^{2^{4}+1} t_{8, j}
$$

It is easy to verify the following

\begin{tabular}{|c|c|c|c|c|c|c|c|c|c|c|c|c|c|c|c|c|}
\hline$t_{8,1}$ & $t_{8,2}$ & $t_{8,3}$ & $t_{8,4}$ & $t_{8,5}$ & $t_{8,6}$ & $t_{8,7}$ & $t_{8,8}$ & $t_{8,9}$ & $t_{8,10}$ & $t_{8,11}$ & $t_{8,12}$ & $t_{8,13}$ & $t_{8,14}$ & $t_{8,15}$ & $t_{8,16}$ & $t_{8,17}$ \\
\hline 0 & 10 & 16 & 22 & 24 & 30 & 32 & 34 & 32 & 38 & 40 & 42 & 40 & 42 & 40 & 38 & 32 \\
\hline
\end{tabular}

Table 3.2: The values of $t_{8, j}$ for $j \in\left[1,2^{4}+1\right]$

Notice that

$$
E\left(U_{1}^{n}\right) \cup E\left[U_{1}^{n}, V_{1}^{n}\right]=H_{\text {black }}^{n} .
$$


By (47), (501), (48), (51), (52), Lemma 2.1, Property A, Assertion C and Table 3.2, we conclude that

$$
\begin{aligned}
& \nu_{\Gamma_{n}}\left(\operatorname{In}\left(\bigcup_{t \in\{-n, n\}} \mathscr{I}_{t}\left(V_{1,1}^{n}\right)\right), \operatorname{In}\left(E\left(U_{1}^{n}\right) \cup E\left[U_{1}^{n}, V_{1}^{n}\right]\right)\right) \\
& =\nu_{\Gamma_{n}}\left(\operatorname{In}\left(\mathscr{I}_{-n}\left(V_{1,1}^{n}\right)\right), \operatorname{In}\left(E\left(U_{1}^{n}\right) \cup E\left[U_{1}^{n}, V_{1}^{n}\right]\right)\right) \\
& +\nu_{\Gamma_{n}}\left(\operatorname{In}\left(\mathscr{I}_{n}\left(V_{1,1}^{n}\right)\right), \operatorname{In}\left(E\left(U_{1}^{n}\right)\right)\right)+\nu_{\Gamma_{n}}\left(\operatorname{In}\left(\mathscr{I}_{n}\left(V_{1,1}^{n}\right)\right), E\left[U_{1,1}^{n}, V_{1,1}^{n}\right]\right) \\
& =\left(\sum_{j=1}^{2^{n-4}} t_{n, j}+\left(t_{n, 2^{n-6}+1}-t_{n, 2^{n-6}}\right)+\left(t_{n, 2^{n-6}+2^{n-7}+1}-t_{n, 2^{n-6}+2^{n-7}}\right)+\left(t_{n, 2^{n-5}+1}-t_{n, 2^{n-5}}\right)\right. \\
& +\left(t_{n, 2^{n-5}+2^{n-7}+1}-t_{n, 2^{n-5}+2^{n-7}}\right)+\left(t_{n, 2^{n-5}+2^{n-6}+1}-t_{n, 2^{n-5}+2^{n-6}}\right) \\
& \left.+\sum_{j=2^{n-5}+2^{n-6}+2}^{2^{n-4}}\left(t_{n, j+1}-t_{n, j}\right)\right) \\
& +\left(2^{n-5} \cdot t_{n, 1}^{\prime}+2^{n-7} \cdot t_{n, 2^{n-5}+1}^{\prime}+2^{n-8} \cdot t_{n, 2^{n-5}+2^{n-7}+1}^{\prime}+2^{n-8} \cdot t_{n, 2^{n-5}+2^{n-6}+1}^{\prime}+2^{n-6} \cdot t_{n, 2^{n-4}+1}^{\prime}\right) \\
& +\left(2 \cdot\left(\begin{array}{c}
2^{n-7} \\
2
\end{array}\right)+2 \cdot 2 \cdot\left(\begin{array}{c}
2^{n-8} \\
2
\end{array}\right)+2 \cdot\left(\begin{array}{c}
2^{n-6} \\
2
\end{array}\right)\right) \\
& =\left(\sum_{j=1}^{2^{n-4}} t_{n, j}+\left(t_{n, 2^{n-6}+1}-t_{n, 2^{n-6}}\right)+\left(t_{n, 2^{n-6}+2^{n-7}+1}-t_{n, 2^{n-6}+2^{n-7}}\right)+\left(t_{n, 2^{n-5}+1}-t_{n, 2^{n-5}}\right)\right. \\
& +\left(t_{n, 2^{n-5}+2^{n-7}+1}-t_{n, 2^{n-5}+2^{n-7}}\right)+\left(t_{n, 2^{n-5}+2^{n-6}+1}-t_{n, 2^{n-5}+2^{n-6}}\right) \\
& \left.+\sum_{j=2^{n-5}+2^{n-6}+2}^{2^{n-4}}\left(t_{n, j+1}-t_{n, j}\right)\right) \\
& +\left(2^{n-5} \cdot t_{n, 1}+2^{n-7} \cdot t_{n, 2^{n-5}+1}+2^{n-8} \cdot t_{n, 2^{n-5}+2^{n-7}+1}+2^{n-8} \cdot t_{n, 2^{n-5}+2^{n-6}+1}+2^{n-6} \cdot t_{n, 2^{n-4}+1}\right) \\
& +\left(2 \cdot\left(\begin{array}{c}
2^{n-7} \\
2
\end{array}\right)+2 \cdot 2 \cdot\left(\begin{array}{c}
2^{n-8} \\
2
\end{array}\right)+2 \cdot\left(\begin{array}{c}
2^{n-6} \\
2
\end{array}\right)\right) \\
& =\left(\sum_{j=1}^{2^{n-4}+1} t_{n, j}+\sum_{j=2}^{6} t_{n, j \times 2^{n-7}+1}-\sum_{j=2}^{6} t_{n, j \times 2^{n-7}}-t_{n, 6 \times 2^{n-7}+2}\right) \\
& +\left(2^{n-7} \cdot t_{n, 4 \times 2^{n-7}+1}+2^{n-8} \cdot t_{n, 5 \times 2^{n-7}+1}+2^{n-8} \cdot t_{n, 6 \times 2^{n-7}+1}+2^{n-6} \cdot t_{n, 8 \times 2^{n-7}+1}\right) \\
& +\left(11 \cdot 2^{2 n-15}-2^{n-5}\right) \\
& =\left(4^{n-8} \sum_{j=1}^{2^{4}+1} t_{8, j}+\sum_{j=2}^{6} 2^{n-8} \cdot t_{8,2 j+1}-\sum_{j=2}^{6}\left(t_{8,2 j}+\left(2^{n-8}-1\right) \cdot t_{8,2 j+1}+2 \cdot(n-8)\right)\right. \\
& \left.-\left(\left(2^{n-8}-1\right) \cdot t_{8,13}+t_{8,14}+2 \cdot(n-8)\right)\right) \\
& +\left(2^{n-7} \cdot 2^{n-8} \cdot t_{8,9}+2^{n-8} \cdot 2^{n-8} \cdot t_{8,11}+2^{n-8} \cdot 2^{n-8} \cdot t_{8,13}+2^{n-6} \cdot 2^{n-8} \cdot t_{8,17}\right) \\
& +\left(11 \cdot 2^{2 n-15}-2^{n-5}\right) \\
& =\left(512 \cdot 2^{2 n-16}+168 \cdot 2^{n-8}-\left(166+168 \cdot\left(2^{n-8}-1\right)+10 \cdot(n-8)\right)\right. \\
& \left.-\left(40 \cdot\left(2^{n-8}-1\right)+42+2 \cdot(n-8)\right)\right) \\
& +\left(32 \cdot 2^{2 n-15}+40 \cdot 2^{n-16}+40 \cdot 2^{2 n-16}+32 \cdot 2^{n-14}\right) \\
& +\left(11 \cdot 2^{2 n-15}-2^{n-5}\right) \\
& =403 \cdot 2^{2 n-15}-3 \cdot 2^{n-4}-12 n+96 \text {. }
\end{aligned}
$$


Therefore, it follows from (105), (106), (107) and (112) that

$$
\begin{aligned}
\nu_{\Gamma_{n}}\left(E_{\text {blue }}^{n}, E_{\text {black }}^{n}\right)=8 \cdot( & \frac{7}{3} \cdot 4^{n-4}-\left(2 n-\frac{14}{3}+\frac{7\left(1+(-1)^{n}\right)}{6}\right) \cdot 2^{n-5} \\
& \quad+403 \cdot 2^{2 n-15}-3 \cdot 2^{n-4}-12 n+96 \\
& \left.\quad+\frac{79}{3} \cdot 2^{2 n-12}-\left(4 n-\frac{29}{3}+\frac{7 \cdot\left(1+(-1)^{n-1}\right)}{6}\right) \cdot 2^{n-6}\right) \\
= & 8 \cdot\left(\frac{2737}{3} \cdot 2^{2 n-15}-\left(8 n-\frac{14}{3}+\frac{7 \cdot\left(1+(-1)^{n}\right)}{6}\right) \cdot 2^{n-6}-12 n+96\right) \\
= & \frac{2737}{3} \cdot 2^{2 n-12}-\left(8 n-\frac{14}{3}+\frac{7 \cdot\left(1+(-1)^{n}\right)}{6}\right) \cdot 2^{n-3}-96 n+768 .
\end{aligned}
$$

By (74), (81), (86), (104), (94) and (113), for $n \geq 8$, we have that

$$
\begin{aligned}
\nu_{\Gamma_{n}}\left(A Q_{n}\right)= & \nu_{\Gamma_{n}}\left(E_{\text {blue }}^{n}\right)+\nu_{\Gamma_{n}}\left(E_{\text {red }}^{n}\right)+\nu_{\Gamma_{n}}\left(E_{\text {black }}^{n}\right) \\
& +\nu_{\Gamma_{n}}\left(E_{\text {red }}^{n}, E_{\text {blue }}^{n}\right)+\nu_{\Gamma_{n}}\left(E_{\text {red }}^{n}, E_{\text {black }}^{n}\right)+\nu_{\Gamma_{n}}\left(E_{\text {blue }}^{n}, E_{\text {black }}^{n}\right) \\
= & \left(11 \cdot 2^{2 n-13}+2^{n-3}\right) \\
& +\left(71 \cdot 2^{2 n-10}-5 \cdot 2^{n-4}\right) \\
& +\left(59 \cdot 2^{2 n-8}-\left(4 n^{2}-9 n-6\right) \cdot 2^{n-3}-7 \cdot\left(1+(-1)^{n-1}\right) \cdot 2^{n-4}\right) \\
& +\left(19 \cdot 2^{2 n-8}-3 \cdot 2^{n-3}-48\right) \\
& +\left(389 \cdot 2^{2 n-11}-(n-1) \cdot 2^{n}+7 \cdot\left(1+(-1)^{n-1}\right) \cdot 2^{n-5}\right) \\
& +\left(\frac{2737}{3} \cdot 2^{2 n-12}-\left(8 n-\frac{14}{3}+\frac{7 \cdot\left(1+(-1)^{n}\right)}{6}\right) \cdot 2^{n-3}-96 n+768\right) \\
= & \frac{19367}{3} \cdot 2^{2 n-13}-\left(8 n^{2}+14 n-\frac{85}{3}\right) \cdot 2^{n-4}-\left(\frac{28}{3}+\frac{7 \cdot\left(1+(-1)^{n-1}\right)}{3}\right) \cdot 2^{n-5}-96 n+720 \\
= & \frac{19367}{3} \cdot 2^{2 n-13}-\left(8 n^{2}+14 n-\frac{71}{3}\right) \cdot 2^{n-4}-\frac{7 \cdot\left(1+(-1)^{n-1}\right)}{3} \cdot 2^{n-5}-96 n+720 \\
< & \frac{19367}{3} \cdot 2^{2 n-13}-\left(8 n^{2}+14 n-\frac{71}{3}\right) \cdot 2^{n-4} \\
< & \frac{19368}{3} \cdot 2^{2 n-13}-\left(8 n^{2}+14 n-\frac{72}{3}\right) \cdot 2^{n-4} \\
= & 807 \cdot 4^{n-5}-\left(4 n^{2}+7 n-12\right) \cdot 2^{n-3} \\
< & \frac{26}{32} \cdot 4^{n}-\left(2 n^{2}+\frac{7}{2} n-6\right) \cdot 2^{n-2} .
\end{aligned}
$$

This completes the proof of Theorem 1.1.

\section{Concluding remarks}

In this section, we make a study of the crossing number of $A Q_{n}$ for $n \leq 7$. It is clear that $\operatorname{cr}\left(A Q_{1}\right)=\operatorname{cr}\left(A Q_{2}\right)=$ 0. In Figure 4.1 (1), we show a drawing of $A Q_{3}$ with 4 crossings, it means $\operatorname{cr}\left(A Q_{3}\right) \leq 4$. Since $A Q_{3}$ contains a subgraph isomorphic to $K_{4,4}$, we have $\operatorname{cr}\left(A Q_{3}\right) \geq \operatorname{cr}\left(K_{4,4}\right)=4$. Hence,

Proposition 4.1. $\operatorname{cr}\left(A Q_{3}\right)=4$.

Meanwhile, in Figure 4.1 (2)-(3), Figure 4.2 and Figure 4.3 we show the drawings of $A Q_{4}, A Q_{5}, A Q_{6}$ and $A Q_{7}$ with 46, 328, 1848 and 9112 crossings, respectively. We remark that the calculations of crossings in the following drawings which are given in Figures 4.1-4.3 are similar to $\nu_{\Gamma_{n}}\left(A Q_{n}\right)$ for $n \geq 8$, and omit them here. Hence, we have the following

Proposition 4.2. $\operatorname{cr}\left(A Q_{4}\right) \leq 46, \operatorname{cr}\left(A Q_{5}\right) \leq 328, \operatorname{cr}\left(A Q_{6}\right) \leq 1848, \operatorname{cr}\left(A Q_{7}\right) \leq 9112$.

In the final of this paper, by just applying the same technique of congestions proposed by Leighton [19], we can obtain the following lower bound:

$$
\operatorname{cr}\left(A Q_{n}\right)>\frac{4^{n}}{5 \times\left(1+2^{2-n}\right)^{2}}-\left(4 n^{2}+4 n+\frac{17}{5}\right) 2^{n-1} .
$$




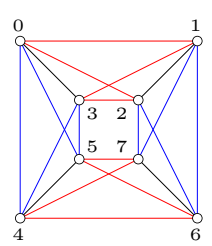

(1) $\operatorname{cr}\left(A Q_{3}\right)=4$

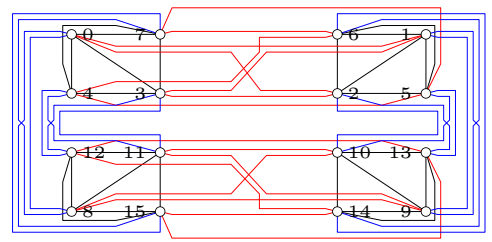

(2) $\operatorname{cr}\left(A Q_{4}\right) \leq 46$

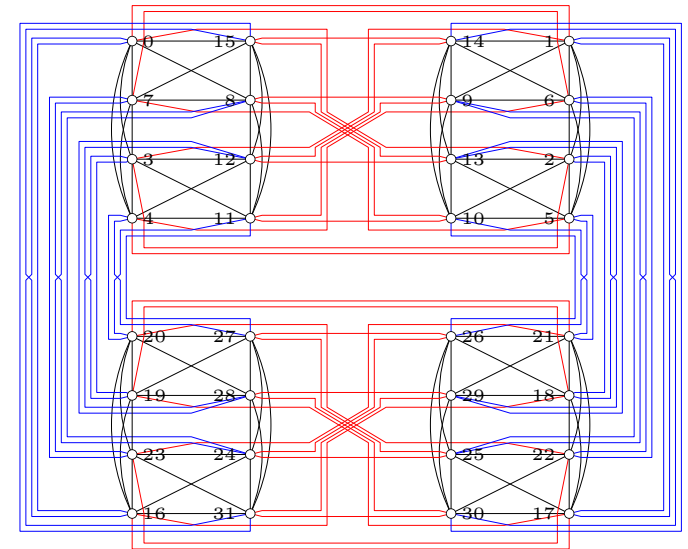

(3) $\operatorname{cr}\left(A Q_{5}\right) \leq 328$

Figure 4.1: Some drawings of $A Q_{3}, A Q_{4}$ and $A Q_{5}$

\section{References}

[1] S.N. Bhatt, F.T. Leighton, A framework for solving VLSI graph layout problems, J. Comput. System Sci. 28 (1984) 300-343.

[2] L. Bhuyan, D.P. Agrawal, Generalized hypercubes and hyperbus structure for a computer network, IEEE Trans. Comput. 33 (1984) 323-333.

[3] H.C. Chan, J.M. Chang, Y.L. Wang, S.J. Horng, Geodesic-pancyclicity and fault-tolerant panconnectivity of augmented cubes, Appl. Math. Comput. 207 (2009) 333-339.

[4] N.W. Chang, S.Y. Hsieh, Conditional diagnosability of augmented cubes under the PMC model, IEEE Transactions on Dependable and Secure Computing 9 (2012) 46-60.

[5] S.A. Choudum, V. Sunitha, Augmented Cubes, Networks 40 (2002) 71-84.

[6] A.M. Dean, R.B. Richter, The crossing number of $C_{4} \times C_{4}$, J. Graph Theory 19 (1995) 125-129.

[7] R.B. Eggleton, R.K. Guy, The crossing number of the n-cube, Notices Amer. Math. Soc. 17 (1970) 757-757.

[8] P. Erdős, R.K. Guy, Crossing number problems, Amer. Math. Monthly 80 (1973) 52-58.

[9] L. Faria, C.M.H. de Figueiredo, On Eggleton and Guy's conjectured upper bound for the crossing number of the $n$-cube, Math. Slovaca 50 (2000) 271-287.

[10] L. Faria, C.M.H. de Figueiredo, O. Sykora, I. Vrt'o, An improved upper bound on the crossing number of the hypercube, J. Graph Theory 59 (2008) 145-159.

[11] M.R. Garey, D.S. Johnson, Crossing number is NP-complete, SIAM J. Alg. Disc. Math. 4 (1983) 312-316.

[12] R.K. Guy, A combinatorial problem, Nabla(Bull. Malayan Math. Soc.) 7 (1960) 68-72.

[13] W.S. Hong, S.Y. Hsieh, Strong diagnosability and conditional diagnosability of augmented cubes under the comparison diagnosis model, IEEE Transactions on Reliability 61 (2012) 140-148.

[14] S.Y. Hsieh, Y.R. Cian, Conditional edge-fault Hamiltonicity of augmented cubes, Inform. Sci. 180 (2010) $2596-2617$.

[15] S.Y. Hsieh, J.Y. Shiu, Cycle embedding of augmented cubes, Appl. Math. Comput. 191 (2007) 314-319.

[16] H.C. Hsu, L.C. Chiang, J.J.M. Tan, L.H. Hsu, Fault hamiltonicity of augmented cubes, Parallel Comput. 31 (2005) 130-145.

[17] H.C. Hsu, P.L. Lai, C.H. Tsai, Geodesic pancyclicity and balanced pancyclicity of augmented cubes, Inform. Process. Lett. 101 (2007) 227-232. 
[18] C.M. Lee, Y.H. Teng, Jimmy J.M. Tanc, L.H. Hsu, Embedding Hamiltonian paths in augmented cubes with a required vertex in a fixed position, Computers and Mathematics with Applications 58 (2009) 1762-1768.

[19] F.T. Leighton, New lower bound techniques for VLSI, Math. Systems Theory 17 (1984) 47-70.

[20] F.T. Leighton, Complexity Issues in VLSI, Found. Comput. Ser., MIT Press, Cambridge, MA (1983).

[21] F.T. Leighton, Introduction to Parallel Algorithms and Architecture: Arrays, Trees, Hypercubes, Morgan Kaufmann, San Mateo, CA (1992).

[22] X.H. Lin, Y.S. Yang, W.P. Zheng, L. Shi, W.M. Lu, The crossing numbers of generalized Petersen graphs with small order, Discrete Appl. Math. 157 (2009) 1016-1023.

[23] M. Ma, G. Liu, J.M. Xu, Panconnectivity and edge-fault-tolerant pancyclicity of augmented cubes, Parallel Comput. 33 (2007) 35-42.

[24] T. Madej, Bounds for the crossing number of the n-cube, J. Graph Theory 15 (1991) 81-97.

[25] S. Pan, R.B. Richter, The crossing number of $K_{11}$ is 100, J. Graph Theory 56 (2007) 128-134.

[26] R.B. Richter, C. Thomassen, Intersections of curve systems and the crossing number of $C_{5} \times C_{5}$, Discrete Comput. Geom. 13 (1995) 149-159.

[27] G. Salazar, On the crossing numbers of loop networks and generalized Petersen graphs, Discrete Math. 302 (2005) 243-253.

[28] O. Sykora, I. Vrt'o, On crossing numbers of hypercubes and cube connected cycles, BIT 33 (1993) $232-237$.

[29] P. Turán, A note of welcome, J. Graph Theory 1 (1977) 7-9.

[30] W.T. Tutte, Toward a theory of crossing numbers, J. Combinatorial Theory 8 (1970) 45-53.

[31] W.W. Wang, M.J. Ma, J.M. Xu, Fault-tolerant pancyclicity of augmented cubes, Inform. Process. Lett. 103 (2007) 52-56.

[32] M. Xu, J.M. Xu, The forwarding indices of augmented cubes, Inform. Process. Lett. 101 (2007) 185-189.

[33] Y.S. Yang, G.Q. Wang, H.L. Wang, Y. Zhou, The Erdős and Guy's conjectured equality on the crossing number of hypercubes, arXiv:1201.4700 1 . 


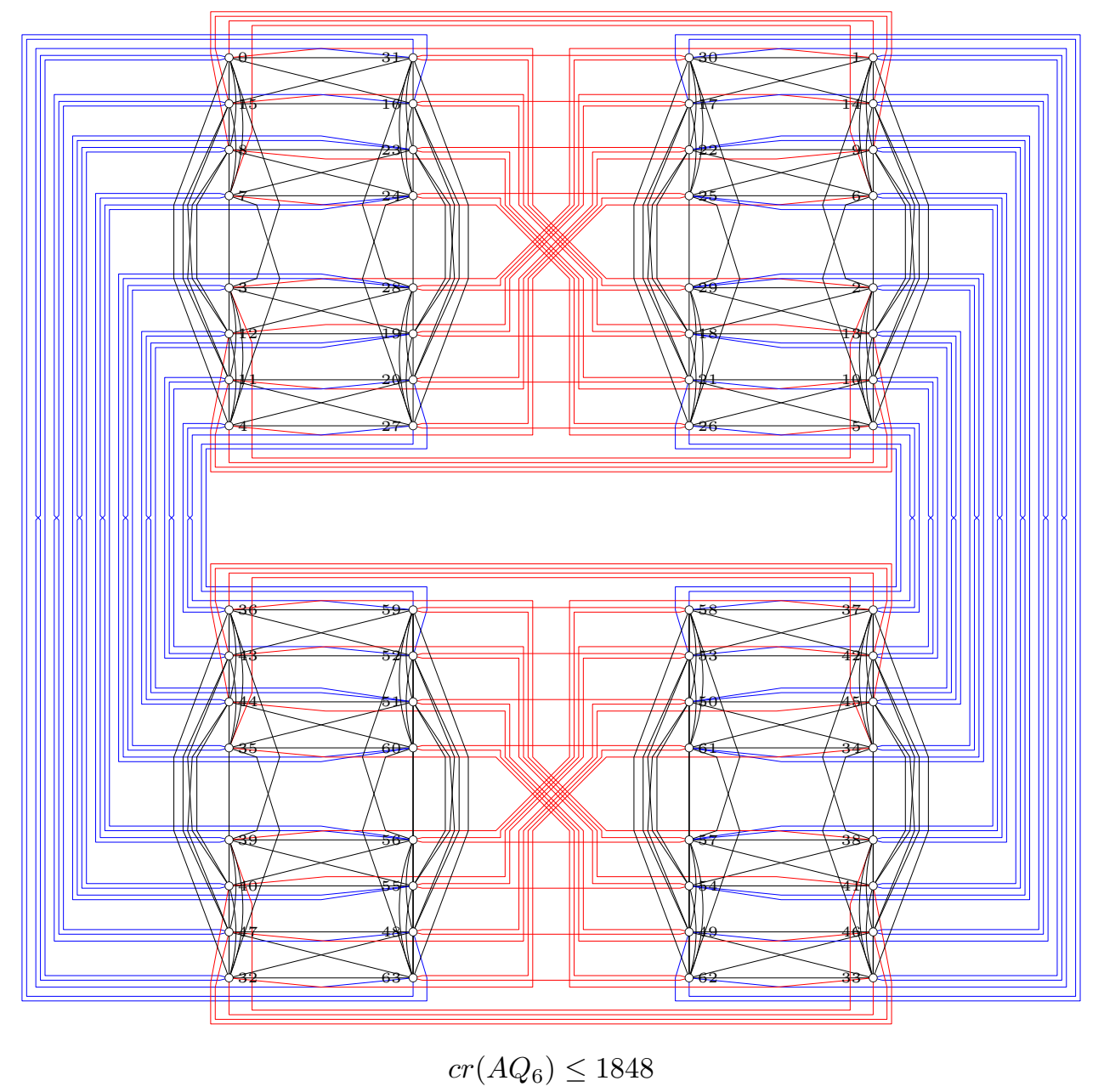

Figure 4.2: A drawing of $A Q_{6}$ with 1848 crossings 


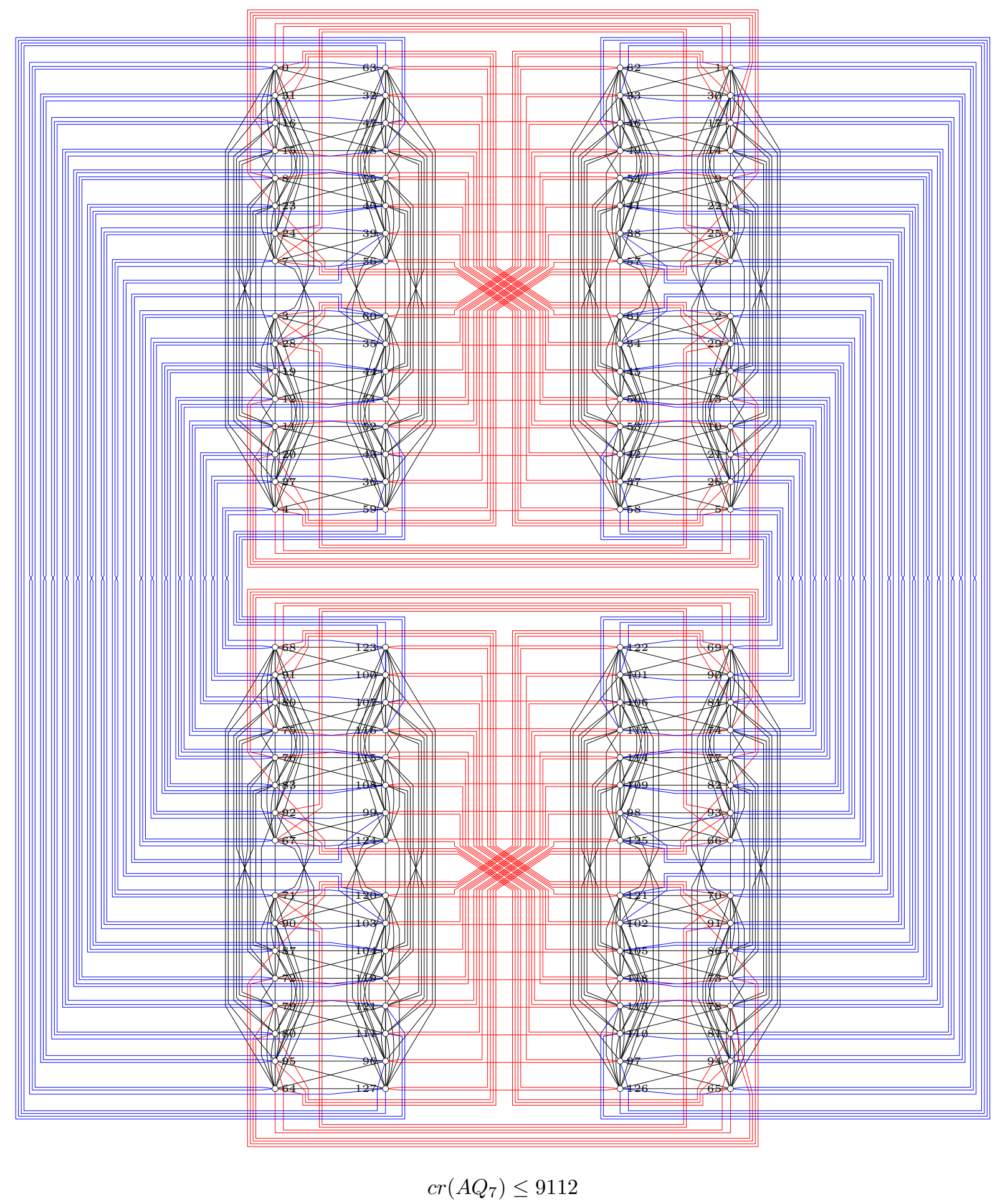

Figure 4.3: A drawing of $A Q_{7}$ with 9112 crossings 